\title{
Jellyfish galaxy candidates in MACS J0717.5+3745 and 39 other clusters of the DAFT/FADA and CLASH surveys ${ }^{\star}$
}

\author{
F. Durret ${ }^{1}$, S. Chiche ${ }^{1}$, C. Lobo ${ }^{2,3}$, and M. Jauzac ${ }^{4,5,6,7}$ \\ 1 Sorbonne Université, CNRS, UMR 7095, Institut d'Astrophysique de Paris, 98bis Bd Arago, 75014 Paris, France \\ e-mail: durret@iap.fr \\ 2 Instituto de Astrofísica e Ciências do Espaço, Universidade do Porto, CAUP, Rua das Estrelas, 4150-762 Porto, Portugal \\ 3 Departamento de Física e Astronomia, Faculdade de Ciências, Universidade do Porto, Rua do Campo Alegre 687, 4169-007 Porto, \\ Portugal \\ ${ }^{4}$ Centre for Extragalactic Astronomy, Durham University, South Road, Durham DH1 3LE, UK \\ 5 Institute for Computational Cosmology, Durham University, South Road, Durham DH1 3LE, UK \\ 6 Astrophysics Research Centre, University of KwaZulu-Natal, Westville Campus, Durban 4041, South Africa \\ 7 School of Mathematics, Statistics \& Computer Science, University of KwaZulu-Natal, Westville Campus, Durban 4041, \\ South Africa
}

Received 27 October 2020 / Accepted 2 February 2021

\begin{abstract}
Context. Galaxies in clusters undergo several phenomena, such as RPS and tidal interactions, that can trigger or quench their star formation and, in some cases, lead to galaxies acquiring unusual shapes and long tails - some become jellyfish.

Aims. We searched for jellyfish galaxy candidates in a sample of 40 clusters from the DAFT/FADA and CLASH surveys covering the redshift range $0.2<z<0.9$. In MACS J0717.5+3745 (MACS0717), our large spatial coverage and abundant sampling of spectroscopic redshifts allowed us to pursue a detailed analysis of jellyfish galaxy candidates in this cluster and its extended filament. Methods. We retrieved galaxy spectroscopic redshifts in the NASA Extragalactic Database for galaxies in all the clusters of our sample (except for MACS0717 for which we had an extensive catalogue), looked at the Hubble Space Telescope ACS images of these objects (mainly the $F 606 \mathrm{~W}$ and $F 814 \mathrm{~W}$ bands), and classified them as a function of their likeliness to be jellyfish galaxies. We give catalogues of jellyfish galaxy candidates with positions, redshifts, magnitudes, and projected distance to their respective cluster centre. For MACS0717, an eight-magnitude optical and infrared catalogue covering the entire region allowed us to compute the best stellar population fits with LePhare through the GAZPAR interface. For the 31 jellyfish candidates in the other clusters belonging to the CLASH survey, we extracted up to 17 magnitudes available in the CLASH catalogues to fit their spectral energy distribution in the same way.

Results. We found 81 jellyfish galaxy candidates in the extended region around MACS0717 as well as 97 in 22 other clusters. Jellyfish galaxy candidates in MACS0717 tend to avoid the densest regions of the cluster, while this does not appear to be the case in the other clusters. The best fit templates found by LePhare show that star formation is occurring. Stellar masses are in the range $10^{9}-10^{11} M_{\odot}$, and the star formation rates (SFRs) are in the $10^{-1}-60 M_{\odot} \mathrm{yr}^{-1}$ range for MACS0717 and in the $10^{-1}-10 M_{\odot} \mathrm{yr}^{-1} \mathrm{range}$ for the other sample. Specific star formation rates (sSFRs) are notably higher in MACS0717, with more than half of the sample having values larger than $10^{-9} \mathrm{yr}^{-1}$, while in the other clusters, most galaxies have SSFR $<10^{-10} \mathrm{yr}^{-1}$. Stellar populations appear younger in MACS0717 (more than half have an age smaller than $1.5 \times 10^{9} \mathrm{yrs}$ ), and, following mid-infrared criteria, two galaxies may contain an active galactic nucleus. In a SFR versus stellar mass diagram, jellyfish galaxy candidates appear to have somewhat larger SFRs than "nonjellyfish star-forming" galaxies. For MACS0717, the mean sSFR of the 79 jellyfish galaxy candidates is 3.2 times larger than that of star-forming non-jellyfish galaxies (selected with $\log (\mathrm{sSFR}) \geq-11$ ).

Conclusions. Our jellyfish galaxy candidates are star-forming objects, with young ages and blue colours. Based on several arguments, the jellyfish candidates identified in MACS0717 seem to have fallen rather recently into the cluster. A very rough estimate of the proportions of jellyfish galaxies in the studied clusters is about $10 \%$; this number does not seem to vary strongly with the cluster relaxation state, though this result must be confirmed with more data. Our sample of 97 galaxies in 22 clusters represents the basis of future works.
\end{abstract}

Key words. galaxies: clusters: general - galaxies: clusters: individual: MACS J0717.5+3745 - galaxies: star formation

\section{Introduction}

Due to the high density of galaxies found in clusters and to the presence of hot X-ray emitting gas, cluster galaxies are subject to environmental mechanisms that do not significantly affect their

\footnotetext{
* Based on archive data of the Hubble Space Telescope. This paper has made use of the NASA Extragalactic Database and of the GAZPAR tool to apply the LePhare software.
}

field counterparts. The most important are ram pressure stripping (RPS; Gunn et al. 1972), hereafter RPS, which affects the gas contained in galaxies, and tidal effects which affect both gas and stars and often lead to harrassment (Moore et al. 1996). A nice summary of all the physical processes taking place in clusters can be found in, for example, the introduction of Poggianti et al. (2017a) and will not be repeated here. We will here concentrate on the description of observations performed these last 15 years by various teams. Galaxies with unusual shapes and star-forming 
properties have been found in many galaxy clusters, mostly at optical wavelengths, but some features (mainly long tails) are also sometimes detected in X-rays and/or at radio wavelengths. The number and depth of the analyses of such objects have been increasing tremendously these last few years, in particular with the advent of the MUSE instrument on the Very Large Telescope (see below). To our knowledge, the first person to name some of these objects "jellyfish galaxies" was Bekki (2009).

Owen et al. (2006) studied the very rich merging cluster Abell $2125(z=0.247)$ at several wavelengths and found galaxy C153; it showed an X-ray plume with [OII] emission in knots, which they interpreted as due to RPS. The spectral energy distribution (SED) of $\mathrm{C} 153$ shows that it has undergone continuous star formation (SF) for $3.5 \mathrm{Gyr}$, further supporting the idea that it can be considered a prototype jellyfish galaxy, even if not named as such. Sun et al. (2007) found ESO137-001 in the massive cluster Abell $3627(z=0.01625)$ with a $40 \mathrm{kpc} \mathrm{H} \alpha$ tail coinciding with a $70 \mathrm{kpc} \mathrm{X}$-ray tail. The $\mathrm{H} \alpha$ emission of the galaxy itself is sharply truncated, and 49 emission line knots are distributed along the tail over $39 \mathrm{kpc}$. These authors attribute the origin of the tail to RPS and noted that heat conduction may contribute to the energy of the optical lines. Cortese et al. (2007) found two peculiar galaxies falling into the massive galaxy clusters Abell $1689(z=0.18)$ and Abell $2667(z=0.23)$. Their Hubble Space Telescope (HST) images show extraordinary trails composed of bright blue knots (with absolute magnitudes in the range $-16.5<M<-11.5$ ) and stellar streams associated with each of these systems, one of which is experiencing a strong burst of SF while the other has recently ceased its SF activity. They interpret these results as being due to the combined action of tidal interaction with the cluster potential and RPS. Yoshida et al. (2008) detected a string of "fireballs" (star-forming clouds with a linear stream of young stars extending towards the galaxy, detected in $\mathrm{H} \alpha$ ) in the Coma cluster "hanging" from galaxy RB 199. They showed that tidal effects alone could not account for the formation of such fireballs but that the RPS mechanism could provide a good explanation. In the optical and UV, Smith et al. (2010) observed 13 star-forming galaxies in the Coma cluster that are asymmetric, due to SF from the gas stripped from the galaxies via interaction with their environment, and long tails reaching $100 \mathrm{kpc}$. Also in Coma, Yagi et al. (2010) found extended $\mathrm{H} \alpha$ clouds in 14 galaxies at the edges of the cluster, suggesting that the parent galaxies have a large velocity with respect to the Coma cluster.

Rawle et al. (2014) analysed SF in 53 galaxies of Abell 2744 $(z=0.308)$, including some jellyfish galaxies. They found that the orientations of the trails, and of the material stripped from constituent galaxies, indicated that the passing shock front of the cluster merger was the trigger. Ebeling et al. (2014) searched for extreme cases of jellyfish galaxies at $z>0.3$ with HST Snapshots and found six very bright objects with $M_{F 606 W}<-21$. They proposed a classification of jellyfish galaxies, from $J=1$ (mildly affected), to $J=5$ (very strongly affected) that is now commonly used. This paper was followed by several others. McPartland et al. (2016) studied 63 MACS clusters and found many more jellyfish galaxies (but with no measured redshifts) showing the presence of optical tails. Their comparison to a simple model showed that extreme RPS events are associated with cluster mergers rather than infall along filaments, even though these do also occur. Kalita \& Ebeling (2019) then detected a showcase jellyfish galaxy in Abell $1758 \mathrm{~N}$ and analysed it in detail, with [OII] emission up to $40 \mathrm{kpc}$. Ebeling \& Kalita (2019) analysed the field of Abell $1758 \mathrm{~N}(z=0.28)$ and detected eight RPS jellyfish candidates undergoing intense SF.
The first paper describing observations of jellyfish galaxies with VLT/MUSE was that of Fumagalli et al. (2014) on ESO 137-001 $(z=0.01625)$. They detected a double tail reaching $80 \mathrm{kpc}$, seen previously in X-rays, and inferred that the galaxy is falling radially into the massive Norma cluster. A complementary study with APEX (Jáchym et al. 2014) had already uncovered an exceptionally long molecular tail in this galaxy; follow-up observations with ALMA (Jáchym et al. 2019) allowed the detection, for the first time, of the molecular gas at the heads of several fireballs located in the complex tail structure of this spectacular galaxy.

Poggianti et al. (2016) published the first analysis of several hundred jellyfish galaxies at low redshift, based on the WINGS + OMEGAWINGS sample, selecting galaxies with various asymmetric/disturbed morphologies and knots, suggestive of triggered SF. This team then obtained a large observing programme on VLT/MUSE: the GAs Stripping Phenomena survey (GASP, Poggianti et al. 2017a). In this first paper of a long series, they show MUSE results on the massive galaxy JO206 $(z=0.0513)$, which is undergoing RPS in a poor cluster and shows a $90 \mathrm{kpc}$ tail. This paper has been followed by many others, out of which we only quote a few. Bellhouse et al. (2019) analysed the $94 \mathrm{kpc}$ long tails of JO201 in Abell 85. George et al. (2019) analysed the galaxy JO201 and showed that this galaxy, which is falling into Abell 85, is located close to the cluster centre, and undergoes both RPS and active galactic nucleus (AGN) feedback. Radovich et al. (2019) analysed seven jellyfish galaxies and highlighted the importance of outflows. Poggianti et al. (2019) achieved a very complete study of JW100 with MUSE and also ALMA, VLA, UVIT, and Chandra, and studied the influence of gas stripping, gas heating, and AGN. They propose that ISM heating due to interaction with the intracluster medium is responsible for the X-ray tail. Moretti et al. (2020) analysed ALMA observations of the jellyfish galaxy JW100, and detected a large amount of molecular gas, $30 \%$ of which is located in the stripped gas tail out to $5 \mathrm{kpc}$ from the galaxy centre. They interpreted this molecular gas, which within the disk is totally displaced relatively to the stellar component, as newly born from stripped HI gas or recently condensed from stripped diffuse molecular gas.

Another interesting result of the GASP survey, obtained to our knowledge for the first time, is that found by Vulcani et al. (2019), who observed four field galaxies with increased SF and tattered $\mathrm{H} \alpha$, making them appear similar to some of our jellyfish galaxies, although they are not members of any cluster. They attributed this increased SF to the effect of cosmic web filaments (none of the galaxies is in a cluster, three are in small groups, and all are embedded in a filament).

At higher redshift, VLT/MUSE results were also obtained by Boselli et al. (2019), who undertook a spectroscopic survey at redshift $0.25<z<0.85$ in groups and clusters (the MAGIC survey: MUSE gAlaxy Groups In Cosmos, Epinat et al., in prep.). They detected two star-forming galaxies in the COSMOS cluster CGr32 at $z=0.73$ with two extended (up to $\sim 100 \mathrm{kpc}$ in projected distance) tails of ionised gas without any stellar counterpart in the deep optical images.

All these studies are devoted to the observations of rather small numbers of jellyfish galaxies per cluster. On the other hand, Roman-Oliveira et al. (2019) observed a large number of jellyfish galaxies (73, out of which they discarded three that were probably interacting galaxies) in a single zone: the multicluster system Abell 901/902 $(z=0.165)$. In particular, they showed that the starburst phenomenon increases with jellyfish class. 
As far as numerical simulations on these specific objects are concerned, Bekki (2009) made hydrodynamical simulations to study the effect of RPS on galaxies in clusters and, to our knowledge, was the first to use the term jellyfish. His simulated galaxies look very much like the jellyfish galaxies that are observed. More recently, Ruggiero et al. (2019) published hydrodynamical simulations to model the four structures observed in Abell 901/902 by Roman-Oliveira et al. (2019). They showed that many (but not all) jellyfish galaxies are located in the vicinity of ram pressure boundaries, defined as regions where gas moving along each sub-cluster and gas from the remainder of the system meet. Galaxies become jellyfish when they cross a boundary within their parent sub-cluster, where a significant pressure increase takes place, due to the merging of the cluster gas and sub-cluster gas. A significant amount of jellyfish galaxies must be created by this mechanism. We can also mention the model by Safarzadeh \& Loeb (2019) accounting for SF due to RPS. According to their results, jellyfish galaxies must be late infallers for their model to work, and they predict no jellyfish galaxies to be present at short clustercentric distances (smaller than $(0.3-0.4) R_{200}$, see their Fig. 3).

We present here a search for jellyfish galaxy candidates in HST images available for clusters of the DAFT/FADA ${ }^{1}$ and $\mathrm{CLASH}^{2}$ surveys. Though a number of such objects have been detected and thoroughly analysed these last years (as discussed before), the number of jellyfish galaxies at medium redshift is still limited, and our aim here is to increase this number in the redshift range $0.2<z<0.9$. For the cluster MACS J0717.5+3745 (hereafter MACS0717) we have a large spectroscopic redshift catalogue that allows us to search for jellyfish galaxies not only in the cluster core but also in its extended filament (Jauzac et al. 2012, 2018a; Durret et al. 2016; Martinet et al. 2016). This will allow a detailed study of the distribution of jellyfish galaxy candidates in this extended environment. For the other clusters, our method will not allow us to make a statistical study since the redshift coverage of the clusters is by no means complete, but it is a first step towards the study of these interesting objects, in particular those at relatively high redshift, and therefore closer to the redshift of cluster formation. The list of new jellyfish galaxy candidates proposed here will hopefully be exploited later at various wavelengths by us or others.

The paper is organized as follows. We describe our initial sample of 40 clusters and the method we apply in Sect. 2. We give our catalogue of 81 jellyfish candidates in the extended region of MACS0717 in Sect. 3, and discuss their spatial distribution and colour. The 97 jellyfish candidates in 22 other clusters (there are 17 clusters in which we found no jellyfish galaxy) are presented in Sect. 4. The SED and derived quantities (such as stellar mass, star formation rate, etc.) of all the jellyfish candidates are analysed in Sect. 5. Finally, we summarise and discuss our results and propose some conclusions in Sect. 6.

All distances are computed with Ned Wright's calculator ${ }^{3}$, assuming $\mathrm{H}_{0}=70 \mathrm{~km} \mathrm{~s}^{-1} \mathrm{Mpc}^{-1}, \Omega_{\Lambda}=0.7$ and $\Omega_{\mathrm{m}}=0.3$. Magnitudes are quoted in $\mathrm{AB}$ system.

\footnotetext{
http://cesam.lam.fr/DAFT/index.php

2 https://archive.stsci.edu/prepds/clash/

http://www. astro.ucla.edu/ wright/CosmoCalc.html
}

\section{Galaxy sample and identification of jellyfish candidates}

\subsection{Selection of jellyfish candidates}

We have considered 40 clusters from the DAFT/FADA and CLASH surveys, which were all selected to be massive clusters $\left(M>2 \times 10^{14} M_{\odot}\right.$ for DAFT/FADA, and $k T>5 \mathrm{keV}$, corresponding to a mass in the $(5-30) \times 10^{14} M_{\odot}$ range, for CLASH). For all clusters except MACS0717, we retrieved from the NASA Extragalactic Database (NED) ${ }^{4}$ all galaxy spectroscopic redshifts available in the regions covered by the HST images. The cluster list is given in Table 1 . The cluster redshift range covered is $0.206 \leq z \leq 0.890$. For MACS0717, the large spectroscopic redshift catalogue available covers not only the cluster core but its extended filament as well, so we dedicate an important part of this paper to its study. The redshift coverage in most clusters is quite homogeneous.

For each cluster, we identify galaxies with spectroscopic redshifts in the range previously chosen to draw galaxy density maps (Durret et al. 2016, 2019) and roughly corresponding to an interval of \pm 0.02 around the corresponding cluster redshift. This translates into a velocity range indicated in Table 1 for each cluster, expressed in units of the corresponding cluster velocity dispersion (computed in Sect. 2.3). We can thus see that the redshift interval first chosen by Durret et al. (2016) is somewhat limited in some cases, and could make us miss fast moving galaxies. In particular for MACS0717, to which a large part of this paper is dedicated, this strategy would limit our analysis to a range of $\pm 2.2 \sigma_{\mathrm{v}}$ (see Fig. 5 of Durret et al. 2016, initial value). We thus decided to extend the redshift interval of this cluster to $\pm 4 \sigma_{\mathrm{v}}$ (final value reported in Table 1), the sole system where we have a large enough spectroscopic and spatial coverage, allowing for a more detailed analysis than what is possible for the remaining clusters.

Two of us (FD and SC) separately looked at each of the selected galaxies, searching for objects that could be classified as jellyfish galaxy candidates based on several criteria: asymmetry, tidal arms, and star trails. We independently classified them between $J=1$ and $J=5$ according to their probability of being a jellyfish, as suggested by Ebeling et al. (2014): $J=1$ being the smallest confidence index and $J=5$ the largest. In most cases, our classifications agreed within \pm 1 , but we prefer to give both classifications, in Tables 2 and 3, to illustrate the relative difficulty of eye classification. We nonetheless favoured eye classification since jellyfish galaxies cover a large variety of shapes, making it difficult to automatize their identification. Out of the 40 clusters considered, there were 17 in which we detected no jellyfish candidate. This is probably due to the fact that in some clusters we only have a small number of redshifts within the imaged area, and in those clusters none of the galaxies with a measured redshift entered this category. We are therefore left with a sample of jellyfish candidates in 22 cluster fields (besides MACS0717).

To visually identify jellyfish galaxies, we used HST images. All clusters apart from C10152.7-1357 have data in the $F 814 \mathrm{~W}$ filter. Whenever possible, we also considered images in the $F 606 W$ filter as well to compare the aspect of the galaxies in both filters. Sometimes, $F 606 \mathrm{~W}$ images were not available, so we considered another filter, as indicated in Table 3 . When possible, we show for each galaxy its images in two filters (with the bluer image to the left and the redder to the right, at the same scale, see Appendix). In some cases, fields covered by the two

\footnotetext{
4 https://ned.ipac.caltech.edu/
} 
Table 1. Clusters studied, ordered by right ascension.

\begin{tabular}{|c|c|c|c|c|c|c|c|c|}
\hline Cluster & $\begin{array}{c}\text { RA (J2000.0) } \\
(\mathrm{deg})\end{array}$ & $\begin{array}{c}\operatorname{Dec}(\mathrm{J} 2000.0) \\
(\mathrm{deg})\end{array}$ & Redshift & $\begin{array}{l}\text { Obs. } \\
\text { gal. }\end{array}$ & $\begin{array}{l}\text { Jelly } \\
\text { cand. }\end{array}$ & $\begin{array}{c}r_{200} \\
(\mathrm{kpc})\end{array}$ & $\begin{array}{c}\sigma_{\mathrm{v}} \\
\left(\mathrm{km} \mathrm{s}^{-1}\right)\end{array}$ & $\Delta v / \sigma_{\mathrm{v}}$ \\
\hline $\mathrm{Cl} 0016+1609$ & 4.64098 & 16.43796 & 0.5410 & 103 & 8 & 1820 & 1034 & \pm 2.7 \\
\hline Abell $209^{C}$ & 22.97083 & -13.60944 & 0.2060 & 39 & 2 & 2410 & 1205 & \pm 2.6 \\
\hline Cl J0152.7-1357 & 28.17083 & -13.96250 & 0.8310 & 66 & 6 & 1670 & 1083 & \pm 2.4 \\
\hline Abell $383^{C}$ & 42.00833 & -3.53750 & 0.1871 & 32 & 2 & 1960 & 973 & \pm 5.0 \\
\hline MACS J0416.1-2403 ${ }^{C}$ & 64.04125 & -24.06611 & 0.3960 & 205 & 8 & 2420 & 1313 & \pm 1.2 \\
\hline MACS J0429.6-0253 ${ }^{C}$ & 67.40000 & -2.88556 & 0.3990 & 2 & 2 & 1730 & 928 & \pm 4.1 \\
\hline MACS J0454.1-0300 & 73.54635 & -3.01494 & 0.5500 & 92 & 5 & 2110 & 1205 & \pm 2.1 \\
\hline MACS J0717.5+3745 & 109.37886 & 37.75826 & 0.5458 & 632 & 81 & 2236 & 1288 & \pm 4.0 \\
\hline MACS J0744.9+3927 & 116.21863 & 39.45759 & 0.6976 & 2 & 0 & & & \\
\hline Abell $611^{C}$ & 120.24542 & 36.04722 & 0.2880 & 2 & 0 & & & \\
\hline Abell 0851 & 145.74167 & 46.98667 & 0.4069 & 102 & 11 & 1542 & 843 & \pm 4.5 \\
\hline LCDCS 0172 & 163.60083 & -11.77167 & 0.6972 & 45 & 9 & 870 & 526 & \pm 2.4 \\
\hline MACS J1115.8+0129 ${ }^{C}$ & 168.96667 & 1.49861 & 0.3520 & 3 & 0 & & & \\
\hline MACS J1149.5+2223 ${ }^{C}$ & 177.39622 & 22.40304 & 0.5444 & 106 & 4 & 2920 & 1665 & \pm 1.4 \\
\hline MACS J1206.2-0847 ${ }^{C}$ & 181.55083 & -8.80028 & 0.4400 & 64 & 3 & 2030 & 1109 & \pm 2.9 \\
\hline BMW-HRI_J122657.3+333253 ${ }^{C}$ & 186.74167 & 3.54836 & 0.8900 & 23 & 0 & & & \\
\hline LCDCS $05 \overline{4} 1$ & 188.12625 & -12.84344 & 0.5414 & 80 & 7 & 860 & 491 & \pm 9.8 \\
\hline MACS J1311.0-0310 & 197.75792 & -3.17667 & 0.4940 & 3 & 0 & & & \\
\hline $\mathrm{ZwCl} 1332.8+5043$ & 203.58500 & 50.51806 & 0.6200 & 6 & 0 & & & \\
\hline [MJM98]_034 & 203.80742 & 37.81564 & 0.3830 & 8 & 1 & & & \\
\hline LCDCS $0829^{C}$ & 206.87750 & -11.75278 & 0.4510 & 35 & 1 & 1638 & 1475 & \pm 1.1 \\
\hline LCDCS 0853 & 208.54083 & -12.51708 & 0.7627 & 20 & 7 & 1590 & 987 & \pm 1.0 \\
\hline 3C 295 CLUSTER & 212.85167 & 52.21056 & 0.4600 & 30 & 8 & 1790 & 984 & \pm 5.4 \\
\hline MACS J1423.8+2404 ${ }^{C}$ & 215.94860 & 24.07782 & 0.5431 & 7 & 0 & & & \\
\hline RX J1524.6+0957 & 231.16792 & 9.96083 & 0.5160 & 2 & 0 & & & \\
\hline $\mathrm{RX} \mathrm{J1532.9+3021^{C }}$ & 233.22417 & 30.34944 & 0.3450 & 2 & 1 & 1630 & 860 & \pm 4.8 \\
\hline RCS J1620.2+2929 & 245.05000 & 29.48333 & 0.8700 & 1 & 0 & & & \\
\hline MACS J1621.4+3810 & 245.35292 & 38.16889 & 0.4650 & 1 & 0 & & & \\
\hline MS $1621.5+2640$ & 245.89792 & 26.57028 & 0.4260 & 31 & 3 & 1718 & 941 & \pm 2.3 \\
\hline OC02 J1701+6412 & 255.34583 & 64.23583 & 0.4530 & 1 & 0 & & & \\
\hline RX J1716.4+6708 & 259.20667 & 67.14167 & 0.8130 & 22 & 1 & 1685 & 1085 & \pm 1.8 \\
\hline MACS J1720.2+3536 & 260.07000 & 35.60722 & 0.3913 & 2 & 0 & & & \\
\hline Abell 2261 & 260.61292 & 32.13389 & 0.2240 & 14 & 0 & & & \\
\hline NEP 0200 & 269.33083 & 66.52528 & 0.6909 & 1 & 0 & & & \\
\hline MACS J1931.8-2634 & 292.95667 & -26.57611 & 0.3520 & 3 & 2 & 1930 & 1018 & \pm 4.0 \\
\hline MS 2053.7-0449 & 314.09083 & -4.63083 & 0.5830 & 32 & 1 & 1620 & 952 & \pm 2.2 \\
\hline MACS J2129.4-0741 ${ }^{C}$ & 322.35922 & -7.69062 & 0.5889 & 2 & 0 & & & \\
\hline MS 2137.3-2353 & 325.06333 & -23.66111 & 0.3130 & 2 & 0 & & & \\
\hline $\mathrm{RXC} \mathrm{J} 2248.7-4431^{C}$ & 342.18125 & -44.52889 & 0.3475 & 42 & 5 & 2300 & 1215 & \pm 3.0 \\
\hline RX J2328.8+1453 & 352.20792 & 14.88667 & 0.4970 & 3 & 0 & & & \\
\hline
\end{tabular}

Notes. Columns are: cluster name (where the subscript $C$ indicates that the cluster comes from the CLASH survey, the other clusters belonging to the DAFT/FADA sample), coordinates, redshift, number of galaxies examined (i.e. galaxies in the cluster redshift range and found in the HST images that we analysed), and number of jellyfish candidates. A zero in the sixth column means that none of the galaxies for which a spectroscopic redshift in the cluster range was available appeared to be a jellyfish candidate. For the clusters in which jellyfish candidates were found, we give in the last three columns the values of $r_{200}, \sigma_{\mathrm{v}}$, and the velocity interval $\Delta v$ in which jellyfish candidates were searched in units of $\sigma_{\mathrm{v}}$ (see text). The last three columns are empty for [MJM98]_034, for which we did not find the information.

filters are not exactly the same, so even if a cluster is observed in two filters, an individual galaxy may be found only in one image. In such cases, as well as for clusters observed in a single band, only one image is shown. For a very small number of cases, the astrometries of the two HST images are slightly different, so images look a little displaced.

However, we must keep in mind that clusters in our sample cover a rather large redshift range, so the rest-frame wavelengths corresponding to the filters analysed are not all the same. For MACS0717 $(z=0.5458)$, the central wavelengths of $F 606 \mathrm{~W}$ and $F 814 W$ filters correspond to rest-frame wavelengths of 392 and $527 \mathrm{~nm}$, respectively. At the extreme redshifts of our sample, at $z=0.2$, the central wavelengths of the $F 606 \mathrm{~W}$ and $F 814 \mathrm{~W}$ filters correspond to rest-frame wavelengths of 505 and $678 \mathrm{~nm}$, respectively, while at $z=0.9$ they correspond to rest frame wavelengths of 310 and $428 \mathrm{~nm}$, respectively.

The selection of jellyfish candidates in MAC0717 followed the same general procedure as outlined here but the dedicated catalogue of magnitudes and redshifts for this specific system introduced some differences that will be described in Sect. 3. 
F. Durret et al.: Jellyfish galaxy candidates in medium redshift clusters

Table 2. Jellyfish candidates in the large structure enclosing the cluster MACS J0717.5+3745.

\begin{tabular}{|c|c|c|c|c|c|c|c|c|c|c|}
\hline Galaxy number & RA & Dec & $z$ & $F 606 W$ & $F 814 W$ & $S$ & $F$ & $\begin{array}{c}\text { Dist. } \\
(\mathrm{kpc})\end{array}$ & $\begin{array}{r}\text { Dist. } \\
\left(r_{200}\right) \\
\end{array}$ & $\Delta v / \sigma_{\mathrm{v}}$ \\
\hline 1 & 109.28460 & 37.76579 & 0.5424 & 21.402 & 20.539 & 3 & 2 & 2453 & 1.097 & -0.43 \\
\hline 2 & 109.29681 & 37.74413 & 0.5445 & 21.605 & 20.526 & 3 & 2 & 2178 & 0.974 & -0.16 \\
\hline $3^{*}$ & 109.30550 & 37.79056 & 0.5416 & 22.103 & 20.638 & 2 & 3 & 2121 & 0.948 & -0.53 \\
\hline 4 & 109.33180 & 37.76147 & 0.5444 & 21.810 & 20.873 & 2 & 1 & 1364 & 0.610 & -0.18 \\
\hline 5 & 109.33253 & 37.76822 & 0.5346 & 22.483 & 21.874 & 1 & 2 & 1372 & 0.613 & -1.41 \\
\hline $6^{*}$ & 109.33324 & 37.68276 & 0.5763 & 22.134 & 21.036 & 1 & 2 & 2134 & 0.955 & 3.76 \\
\hline 7 & 109.33848 & 37.80727 & 0.5350 & 26.239 & 21.237 & 2 & 3 & 1692 & 0.757 & -1.36 \\
\hline 8 & 109.35336 & 37.71206 & 0.5459 & 22.138 & 20.870 & 3 & 3 & 1320 & 0.590 & 0.01 \\
\hline 9 & 109.35354 & 37.73134 & 0.5377 & -99.000 & 22.869 & 4 & 4 & 1022 & 0.457 & -1.02 \\
\hline 10 & 109.37781 & 37.71374 & 0.5374 & 21.923 & 20.766 & 3 & 3 & 1007 & 0.450 & -1.06 \\
\hline 11 & 109.37863 & 37.78915 & 0.5754 & 22.365 & 21.570 & 5 & 5 & 822 & 0.367 & 3.65 \\
\hline 12 & 109.38213 & 37.72594 & 0.5315 & 22.126 & 21.245 & 4 & 4 & 710 & 0.317 & -1.81 \\
\hline $13^{*}$ & 109.38460 & 37.73306 & 0.5325 & 22.111 & 21.418 & 1 & 2 & 537 & 0.240 & -1.68 \\
\hline 14 & 109.39410 & 37.79197 & 0.5757 & 22.878 & 22.084 & 2 & 2 & 840 & 0.376 & 3.68 \\
\hline 15 & 109.39528 & 37.84684 & 0.5348 & 21.759 & 20.512 & 1 & 1 & 2101 & 0.940 & -1.39 \\
\hline 16 & 109.39620 & 37.76458 & 0.5490 & 22.027 & 21.231 & 3 & 2 & 241 & 0.108 & 0.40 \\
\hline 17 & 109.40541 & 37.70779 & 0.5290 & 22.269 & 21.554 & 1 & 1 & 1148 & 0.514 & -2.13 \\
\hline 18 & 109.40749 & 37.61744 & 0.5456 & 22.097 & 21.340 & 4 & 4 & 3199 & 1.431 & -0.03 \\
\hline 19 & 109.40773 & 37.62261 & 0.5459 & 21.055 & 20.251 & 2 & 2 & 3082 & 1.378 & 0.01 \\
\hline $20^{*}$ & 109.41479 & 37.71172 & 0.5395 & 21.618 & 20.502 & 2 & 4 & 1149 & 0.514 & -0.79 \\
\hline 21 & 109.41522 & 37.72866 & 0.5395 & 22.709 & 21.719 & 3 & 2 & 835 & 0.373 & -0.79 \\
\hline 22 & 109.41720 & 37.70310 & 0.5420 & 22.270 & 21.353 & 4 & 4 & 1350 & 0.604 & -0.48 \\
\hline 23 & 109.41817 & 37.79837 & 0.5634 & 22.225 & 20.565 & 4 & 3 & 1168 & 0.522 & 2.18 \\
\hline 24 & 109.42203 & 37.74784 & 0.5660 & 22.493 & 21.425 & 3 & 2 & 768 & 0.343 & 2.50 \\
\hline 25 & 109.42928 & 37.67791 & 0.5442 & 20.739 & 19.954 & 3 & 2 & 1992 & 0.891 & -0.20 \\
\hline 26 & 109.42990 & 37.78072 & 0.5622 & 22.227 & 21.308 & 3 & 3 & 1068 & 0.478 & 2.04 \\
\hline 27 & 109.43111 & 37.62285 & 0.5472 & 21.150 & 20.410 & 2 & 2 & 3189 & 1.426 & 0.18 \\
\hline 28 & 109.43424 & 37.75050 & 0.5500 & 22.224 & 20.866 & 1 & 2 & 1005 & 0.449 & 0.52 \\
\hline 29 & 109.43758 & 37.72152 & 0.5409 & 22.502 & 21.217 & 2 & 0 & 1330 & 0.595 & -0.62 \\
\hline 30 & 109.43866 & 37.79811 & 0.5384 & 21.801 & 20.199 & 1 & 0 & 1472 & 0.658 & -0.93 \\
\hline 31 & 109.44090 & 37.77155 & 0.5521 & 22.692 & 21.920 & 3 & 1 & 1209 & 0.540 & 0.79 \\
\hline 32 & 109.44299 & 37.72516 & 0.5429 & 20.538 & 19.928 & 1 & 0 & 1388 & 0.621 & -0.36 \\
\hline 33 & 109.44773 & 37.63008 & 0.5469 & 22.637 & 21.694 & 3 & 2 & 3168 & 1.417 & 0.14 \\
\hline 34 & 109.44783 & 37.68979 & 0.5577 & 21.773 & 21.141 & 2 & 3 & 2001 & 0.895 & 1.48 \\
\hline 35 & 109.45184 & 37.61336 & 0.5475 & 22.227 & 21.117 & 2 & 1 & 3558 & 1.591 & 0.21 \\
\hline 36 & 109.45511 & 37.64426 & 0.5404 & 21.156 & 20.171 & 2 & 2 & 2955 & 1.322 & -0.68 \\
\hline 37 & 109.45564 & 37.62384 & 0.5480 & 21.919 & 21.650 & 2 & 2 & 3375 & 1.510 & 0.28 \\
\hline 38 & 109.45809 & 37.75248 & 0.5546 & 22.010 & 21.090 & 4 & 2 & 1548 & 0.692 & 1.10 \\
\hline 39 & 109.45857 & 37.61006 & 0.5511 & 21.694 & 20.937 & 5 & 4 & 3690 & 1.650 & 0.66 \\
\hline 40 & 109.46005 & 37.73063 & 0.5401 & 21.537 & 20.933 & 5 & 2 & 1692 & 0.757 & -0.72 \\
\hline 41 & 109.46016 & 37.58284 & 0.5439 & 21.075 & 20.242 & 4 & 1 & 4279 & 1.914 & -0.24 \\
\hline 2 & 109.46469 & 37.61392 & 0.5495 & 22.426 & 21.308 & 3 & 3 & 3673 & 1.643 & 0.46 \\
\hline 43 & 109.46571 & 37.79222 & 0.5515 & 22.613 & 21.842 & 2 & 2 & 1917 & 0.857 & 0.71 \\
\hline 44 & 109.46701 & 37.70718 & 0.5319 & 22.287 & 21.646 & 5 & 2 & 2075 & 0.928 & -1.76 \\
\hline 45 & 109.47195 & 37.61126 & 0.54703 & 21.526 & 20.278 & 1 & 0 & 3806 & 1.702 & 0.15 \\
\hline 46 & 109.47451 & 37.70863 & 0.5311 & 21.922 & 20.994 & 3 & 2 & 2206 & 0.987 & -1.86 \\
\hline 47 & 109.47494 & 37.77757 & 0.5451 & 21.181 & 20.115 & 3 & 0 & 1999 & 0.894 & -0.09 \\
\hline 48 & 109.47574 & 37.71760 & 0.5440 & 22.315 & 19.658 & 4 & 2 & 2139 & 0.956 & -0.23 \\
\hline 49 & 109.48136 & 37.74231 & 0.5637 & 21.287 & 20.237 & 2 & 0 & 2104 & 0.941 & 2.22 \\
\hline 50 & 109.48801 & 37.72609 & 0.5362 & 22.385 & 21.872 & 4 & 5 & 2335 & 1.044 & -1.21 \\
\hline 51 & 109.48879 & 37.55154 & 0.5293 & 22.061 & 21.185 & 4 & 3 & 5204 & 2.327 & -2.09 \\
\hline 52 & 109.49406 & 37.56277 & 0.55338 & 22.452 & 21.247 & 2 & 0 & 5028 & 2.249 & 0.95 \\
\hline 53 & 109.49385 & 37.64037 & 0.5461 & 22.405 & 21.182 & 2 & 3 & 3553 & 1.589 & 0.04 \\
\hline 54 & 109.49413 & 37.59956 & 0.5464 & 22.503 & 20.107 & 5 & 2 & 4302 & 1.924 & 0.07 \\
\hline $55^{*}$ & 109.50267 & 37.65858 & 0.5772 & 22.103 & 21.021 & 4 & 3 & 3404 & 1.522 & 3.86 \\
\hline 56 & 109.50533 & 37.63641 & 0.5412 & 20.762 & 19.552 & 3 & 1 & 3800 & 1.699 & -0.58 \\
\hline 57 & 109.50554 & 37.61765 & 0.5426 & 22.114 & 21.392 & 3 & 3 & 4125 & 1.845 & -0.40 \\
\hline 58 & 109.50652 & 37.67290 & 0.5430 & 21.256 & 20.058 & 1 & 0 & 3269 & 1.462 & -0.35 \\
\hline 59 & 109.50808 & 37.64077 & 0.5420 & 21.681 & 20.739 & 3 & 2 & 3773 & 1.687 & -0.48 \\
\hline 60 & 109.50873 & 37.62831 & 0.5425 & 23.489 & 21.638 & 2 & 2 & 3989 & 1.784 & -0.41 \\
\hline 61 & 109.50883 & 37.63732 & 0.5481 & 21.519 & 20.357 & 2 & 2 & 3841 & 1.718 & 0.29 \\
\hline 62 & 109.52255 & 37.55546 & 0.5465 & 22.372 & 21.748 & 4 & 2 & 5508 & 2.463 & 0.09 \\
\hline 63 & 109.53121 & 37.63280 & 0.5421 & 22.468 & 21.475 & 4 & 4 & 4288 & 1.918 & -0.47 \\
\hline 64 & 109.53217 & 37.58715 & 0.5447 & 21.959 & 21.064 & 5 & 4 & 5055 & 2.261 & -0.14 \\
\hline 65 & 109.53279 & 37.55027 & 0.5495 & 21.663 & 20.238 & 1 & 0 & 5739 & 2.567 & 0.46 \\
\hline $66^{*}$ & 109.53319 & 37.64305 & 0.5478 & 21.084 & 20.302 & 3 & 2 & 4172 & 1.866 & 0.25 \\
\hline 67 & 109.53738 & 37.57487 & 0.5513 & 21.634 & 20.531 & 5 & 2 & 5349 & 2.392 & 0.69 \\
\hline
\end{tabular}

Notes. Columns are: galaxy number, RA, Dec, redshift, $F 606 \mathrm{~W}$ and $F 814 \mathrm{~W}$ magnitudes, jellyfish classifications $S$ and $F$ by two of the authors ( $\mathrm{SC}$ and FD), projected distance to cluster centre in $\mathrm{kpc}$ and in units of $r_{200}$, and velocity relative to cluster centre divided by cluster velocity dispersion. 
Table 2. continued.

\begin{tabular}{lrrrrrrrrrr}
\hline \hline Galaxy number & RA & Dec & $z$ & $F 606 W$ & $F 814 W$ & $S$ & $F$ & $\begin{array}{r}\text { Dist. } \\
(\mathrm{kpc})\end{array}$ & $\begin{array}{r}\text { Dist. } \\
\left(r_{200}\right)\end{array}$ & $\Delta v / \sigma_{\mathrm{v}}$ \\
\hline 68 & 109.53812 & 37.68567 & 0.5422 & 23.393 & 23.081 & 2 & 2 & 3749 & 1.676 & -0.45 \\
69 & 109.54137 & 37.70054 & 0.5419 & 21.532 & 20.190 & 1 & 0 & 3685 & 1.648 & -0.49 \\
70 & 109.55122 & 37.64091 & 0.5701 & 22.283 & 21.443 & 3 & 2 & 4533 & 2.027 & 3.00 \\
71 & 109.55586 & 37.57695 & 0.5500 & 21.673 & 20.747 & 3 & 1 & 5592 & 2.501 & 0.52 \\
$72^{*}$ & 109.55758 & 37.68818 & 0.5419 & 22.206 & 21.046 & 3 & 2 & 4135 & 1.849 & -0.49 \\
73 & 109.56507 & 37.57967 & 0.5488 & 21.814 & 20.612 & 4 & 2 & 5693 & 2.546 & 0.38 \\
74 & 109.57690 & 37.67111 & 0.5236 & 22.552 & 21.855 & 2 & 3 & 4698 & 2.101 & -2.82 \\
75 & 109.58230 & 37.62237 & 0.5625 & 21.298 & 20.379 & 4 & 0 & 5363 & 2.398 & 2.07 \\
76 & 109.58243 & 37.60096 & 0.5449 & 22.312 & 21.025 & 2 & 2 & 5661 & 2.532 & -0.11 \\
77 & 109.58437 & 37.68565 & 0.5482 & 21.371 & 20.508 & 2 & 0 & 4732 & 2.116 & 0.30 \\
78 & 109.58499 & 37.61001 & 0.5489 & 22.352 & 21.587 & 5 & 4 & 5579 & 2.495 & 0.39 \\
79 & 109.59241 & 37.65249 & 0.5378 & 24.508 & 21.100 & 4 & 3 & 5206 & 2.328 & -1.01 \\
80 & 109.60329 & 37.59384 & 0.5542 & 21.376 & 20.345 & 4 & 4 & 6139 & 2.746 & 1.05 \\
81 & 109.60480 & 37.64520 & 0.5225 & 22.826 & 22.159 & 3 & 2 & 5536 & 2.476 & -2.96 \\
\hline
\end{tabular}

We must note that, except for MACS0717, our study relies on spectroscopic redshifts gathered from NED, which are not complete in any way. We will therefore obtain some indications, but will not be able to obtain statistically meaningful results, so this search is mainly a basis for future studies.

Jellyfish galaxies are mainly accounted for by hydrodynamical interactions (RPS) with the hot intracluster gas, which causes various observable effects on the galaxy gas, such as compression of the leading edge of the galaxy, trailing tails, or even unwinding of spiral arms (Bellhouse et al. 2021). On the other hand, gravitational effects (tidal effects, harrassment) impact both gas and stars and can lead to galaxy shapes that can be reminiscent of those of jellyfish galaxies. It is therefore important to spot jellyfish candidates that may be undergoing tidal effects from a neighbour galaxy. For this, we examined all the images of jellyfish candidates and searched for galaxies located within a distance of $50 \mathrm{kpc}$. Out of the 90 (in MACS0717) and 103 (in the other clusters) jellyfish galaxy candidates that we initially identified, nine (in MACS0717) and six (in other clusters) showed actual evidence for gravitational interaction (tidal arms), so they were eliminated from our sample. Among the remaining ones, five jellyfish candidates (in MACS0717) and 18 (in the other clusters) had possible companions but without any signature of interaction with the jellyfish candidate. We kept these galaxies but mark them in Tables 2 and 3 with an asterisk. Our final sample therefore includes 81 jellyfish candidates in MACS0717, and 97 in the 22 other clusters.

\subsection{Galaxy magnitudes}

In order to obtain magnitude measurements for our candidate jellyfish galaxies, we proceeded as follows. For all CLASH clusters except for MACS0717, we retrieved the corresponding catalogues from the CLASH website that contain up to 17 wavebands, between $225 \mathrm{~nm}$ and $1.6 \mu \mathrm{m}$. Some of these magnitudes are given in Table 3, to help characterise the galaxies. The CLASH magnitudes are corrected for Galactic extinction, and can therefore be straightforwardly used for SED analyses (Sect. 5.2.3).

For galaxies from the DAFT/FADA survey that are not part of CLASH, we computed the zero points $\mathrm{ZP}_{A B}$ applying the HST formula: ${ }^{5}$

\footnotetext{
5 http://www.stsci.edu/hst/instrumentation/acs/ data-analysis/zeropoints
}

$-2.5 * \log 10($ PHOTFLAM $)-5 * \log 10($ PHOTPLAM $)-2.408$,

where the PHOTFLAM and PHOTPLAM values were found in the image headers. We then ran SExtractor (Bertin \& Arnouts 1996) on individual images and retrieved the MAG_AUTO magnitudes. The values given in Table 1 are corrected for Galactic extinction computed from the $E(B-V)$ maps by Schlegel et al. (1998) multiplied by the R values given in Table 6 of Schlafly \& Finkbeiner (2011).

For MACS0717, the $F 606 W$ and $F 814 W$ magnitudes for the entire mosaic of images of MACS0717 were taken from the data by Martinet et al. (2017) (we did not use the CLASH catalogue available in the $F 606 W$ and $F 814 W$ filters for this cluster because it only covers the central region). We also used an eight-band ground-based optical and infrared catalogue for the whole zone covered by MACS0717 and its filament with Subaru/SuprimeCam data in the $B, V, R_{c}, I_{c}$ and $z$ bands, CFHT/MegaCam data in the $u^{*}$ band, and CFHT/WIRCAM data in the near-infrared $J$ and $K_{s}$ bands from Jauzac et al. (2012). More details can be found in Ma et al. (2008, 2009).

This eight-magnitude catalogue for MACS0717 as well as the 17 CLASH magnitudes for all the other CLASH clusters were used to fit the SEDs of the jellyfish galaxies, and analyse their main stellar populations, with LePhare (Ilbert et al. 2006), through the GAZPAR facility ${ }^{6}$ as reported in Sect. 5. We also analysed the stellar populations of non-jellyfish galaxies in MACS0717, in order to compare the properties of our jellyfish candidates with those of normal galaxies.

\subsection{Cluster radii and velocity dispersions}

In order to calculate the projected distances of jellyfish galaxy candidates in units linked to the cluster properties, we compute for each cluster its $r_{200}$ value, corresponding to the radius at which the cluster density is 200 times the mean density of the Universe. We did this in several ways. For the seven clusters studied by Martinet et al. (2016), we directly take $r_{200}$ from this paper. For the other clusters, we compute $r_{200}$ from the $M_{200}$ mass. Nine clusters have $M_{200}$ values in Umetsu et al. (2018), and for the remaining clusters (except one, MJM98, for which we cannot find a mass in the literature), we take the $M_{200}$ masses derived from X-ray masses by Chu et al. (2021). We then calculate $r_{200}$ by applying the following formula (Biviano et al. 2013):

\footnotetext{
6 https://gazpar.lam. fr/home
} 
F. Durret et al.: Jellyfish galaxy candidates in medium redshift clusters

Table 3. 97 candidate jellyfish galaxies in 22 clusters.

\begin{tabular}{|c|c|c|c|c|c|c|c|c|c|c|c|c|c|}
\hline Cluster & G. & RA & Dec & $z$ & $F 555 \mathrm{~W}$ & $F 606 W$ & $\begin{array}{l}F 702 W \\
F 775 W\end{array}$ & $F 814 W$ & $F$ & $S$ & $\begin{array}{l}\text { Dist. } \\
(\mathrm{kpc})\end{array}$ & $\begin{array}{r}\text { Dist. } \\
\left(r_{200}\right) \\
\end{array}$ & $\Delta v / \sigma_{\mathrm{v}}$ \\
\hline \multirow[t]{8}{*}{$\mathrm{Cl} 0016+16$} & $\mathrm{a}$ & 4.62890 & 16.42794 & 0.5561 & & & & 19.824 & 4 & 4 & 361 & 0.198 & 2.35 \\
\hline & $\mathrm{b}$ & 4.63388 & 16.42250 & 0.5498 & & & & 20.742 & 3 & 3 & 391 & 0.215 & 1.37 \\
\hline & $\mathrm{c}$ & 4.63674 & 16.43428 & 0.5382 & & & & 21.770 & 2 & 1 & 129 & 0.071 & -0.44 \\
\hline & d & 4.64164 & 16.44986 & 0.56 & & & & 22.033 & 5 & 5 & 274 & 0.151 & 2.95 \\
\hline & $\mathrm{e}^{*}$ & 4.65058 & 16.44316 & 0.5469 & & & & 22.291 & 2 & 1 & 251 & 0.138 & 0.92 \\
\hline & $\mathrm{f}$ & 4.65223 & 16.42086 & 0.5555 & & & & 21.628 & 2 & 3 & 471 & 0.259 & 2.26 \\
\hline & $\mathrm{g}$ & 4.65749 & 16.44081 & 0.54 & & & & 24.100 & 2 & 2 & 385 & 0.212 & -0.16 \\
\hline & $\mathrm{h}$ & 4.66072 & 16.45040 & 0.5642 & & & & 19.872 & 3 & 5 & 536 & 0.295 & 3.59 \\
\hline \multirow[t]{2}{*}{ Abell 209} & $\mathrm{a}$ & 22.95776 & -13.60326 & 0.2123 & & 19.967 & & 19.283 & 2 & 2 & 176 & 0.073 & 1.25 \\
\hline & $b^{*}$ & 22.98058 & -13.60446 & 0.2169 & & 19.103 & & 18.821 & 4 & 4 & 133 & 0.055 & 2.16 \\
\hline \multirow[t]{6}{*}{ Cl 0152.7-1357 } & $\mathrm{a}^{*}$ & 28.12674 & -13.95393 & 0.8474 & & & 21.457 & & 2 & 4 & 1229 & 0.736 & 1.74 \\
\hline & $\mathrm{b}$ & 28.15464 & -13.95295 & 0.8458 & & & 22.763 & & 2 & 4 & 514 & 0.308 & 1.57 \\
\hline & $\mathrm{c}$ & 28.15784 & -13.93587 & 0.8456 & & & 21.338 & & 2 & 4 & 811 & 0.486 & 1.55 \\
\hline & $\mathrm{d}$ & 28.15924 & -13.92740 & 0.8360 & & & 21.583 & & 1 & 2 & 1012 & 0.606 & -1.02 \\
\hline & $\mathrm{e}$ & 28.17714 & -13.93879 & 0.8215 & & & 21.321 & & 2 & 1 & 672 & 0.402 & -1.02 \\
\hline & $\mathrm{f}$ & 28.17842 & -13.96492 & 0.8371 & & & 23.681 & & 2 & 1 & 218 & 0.131 & 0.65 \\
\hline \multirow[t]{2}{*}{ Abell 383} & $\mathrm{a}$ & 42.01002 & -3.55678 & 0.1944 & & 19.487 & & 18.675 & 1 & 3 & 218 & 0.111 & 1.83 \\
\hline & $\mathrm{b}$ & 42.03447 & -3.52755 & 0.1914 & & 19.017 & & 18.311 & 2 & 2 & 315 & 0.161 & 1.08 \\
\hline \multirow[t]{8}{*}{ MACS J0416.1-2403 } & $\mathrm{a}^{*}$ & 64.01709 & -24.08955 & 0.396 & & 20.756 & & 19.761 & 1 & 4 & 647 & 0.267 & 0.00 \\
\hline & $b^{*}$ & 64.01919 & -24.09614 & 0.3844 & & 22.579 & & 21.676 & 1 & 1 & 716 & 0.296 & -0.32 \\
\hline & $\mathrm{c}$ & 64.02498 & -24.09166 & 0.3944 & & 21.533 & & 20.844 & 0 & 1 & 582 & 0.241 & -0.32 \\
\hline & $\mathrm{d}$ & 64.02779 & -24.06101 & 0.3918 & & 22.618 & & 21.893 & 1 & 2 & 277 & 0.114 & -0.62 \\
\hline & $\mathrm{e}$ & 64.03268 & -24.07015 & 0.3973 & & 21.410 & & 20.506 & 2 & 2 & 182 & 0.075 & 0.19 \\
\hline & $\mathrm{f}$ & 64.04131 & -24.07134 & 0.3990 & & 20.676 & & 19.707 & 2 & 3 & 101 & 0.042 & 0.44 \\
\hline & $\mathrm{g}^{*}$ & 64.04419 & -24.06872 & 0.4071 & & 21.319 & & 20.478 & 2 & 4 & 76 & 0.031 & 1.62 \\
\hline & $\mathrm{h}$ & 64.05088 & -24.06027 & 0.3952 & & 22.508 & & 21.874 & 3 & 3 & 217 & 0.089 & -0.12 \\
\hline \multirow[t]{2}{*}{ MACS J0429.6-0253 } & $\mathrm{a}$ & 67.38860 & -2.88379 & 0.4 & & 20.798 & & 20.505 & 4 & 4 & 223 & 0.129 & 0.21 \\
\hline & b & 67.41844 & -2.88832 & 0.4049 & & 20.920 & & 20.665 & 4 & 3 & 360 & 0.208 & 1.22 \\
\hline \multirow{5}{*}{ MACS J0454.1-0300 } & $\mathrm{a}$ & 73.51869 & -3.00396 & 0.5323 & & & & 19.910 & 5 & 5 & 679 & 0.322 & -2.38 \\
\hline & $\mathrm{b}$ & 73.53999 & -3.01686 & 0.5483 & & & & 20.118 & 1 & 2 & 152 & 0.072 & -0.23 \\
\hline & $\mathrm{c}^{*}$ & 73.55029 & -3.00036 & 0.5469 & & & & 20.338 & 1 & 2 & 345 & 0.163 & -0.41 \\
\hline & $\mathrm{d}^{*}$ & 73.56299 & -3.00639 & 0.524 & & & & 22.475 & 5 & 3 & 427 & 0.202 & -3.52 \\
\hline & $\mathrm{e}$ & 73.56342 & -2.99819 & 0.528 & & & & 20.116 & 1 & 2 & 546 & 0.259 & -2.97 \\
\hline \multirow[t]{11}{*}{ Abell 851} & $\mathrm{a}$ & 145.68898 & 46.98263 & 0.407 & & & & 20.668 & 2 & 2 & 1033 & 0.670 & 0.02 \\
\hline & $\mathrm{b}$ & 145.69411 & 47.00624 & 0.4083 & & & & 20.092 & 2 & 1 & 1005 & 0.652 & 0.32 \\
\hline & $\mathrm{c}$ & 145.71105 & 47.01366 & 0.3958 & & & & 19.165 & 5 & 5 & 798 & 0.517 & -2.52 \\
\hline & d & 145.73241 & 47.01542 & 0.3972 & & & & 22.378 & 3 & 3 & 590 & 0.383 & -2.20 \\
\hline & $\mathrm{e}^{*}$ & 145.73256 & 46.99261 & 0.4076 & & & & 19.485 & 3 & 4 & 213 & 0.138 & 0.16 \\
\hline & $\mathrm{f}$ & 145.73862 & 46.96882 & 0.4084 & & & & 20.286 & 2 & 4 & 354 & 0.230 & 0.34 \\
\hline & $\mathrm{g}$ & 145.74384 & 46.99646 & 0.4200 & & & & 21.317 & 1 & 2 & 196 & 0.127 & 2.93 \\
\hline & $\mathrm{h}$ & 145.74773 & 47.01602 & 0.3937 & & & & 21.002 & 4 & 3 & 586 & 0.380 & -3.00 \\
\hline & $\mathrm{i}$ & 145.75700 & 47.00907 & 0.4061 & & & & 19.160 & 2 & 3 & 531 & 0.344 & -0.18 \\
\hline & $\mathrm{j}$ & 145.76435 & 46.98741 & 0.41 & & & & 20.839 & 2 & 3 & 444 & 0.288 & 0.70 \\
\hline & $\mathrm{k}$ & 145.76440 & 47.00949 & 0.39 & & & & 21.286 & 1 & 2 & 630 & 0.408 & -3.85 \\
\hline LCDCS 0172 & $\mathrm{a}$ & 163.57599 & -11.78999 & 0.6965 & & & & 22.109 & 2 & 3 & 793 & 0.911 & -0.18 \\
\hline & $\mathrm{b}$ & 163.58247 & -11.77602 & 0.6972 & & & & 21.651 & 3 & 3 & 485 & 0.557 & 0.00 \\
\hline & $\mathrm{c}$ & 163.58701 & -11.74937 & 0.6968 & & & & 22.337 & 4 & 3 & 674 & 0.774 & -0.10 \\
\hline & $\mathrm{d}$ & 163.58728 & -11.75391 & 0.702 & & & & 20.863 & 3 & 3 & 574 & 0.659 & 1.23 \\
\hline & $\mathrm{e}$ & 163.60573 & -11.76516 & 0.6977 & & & & 20.148 & 1 & 2 & 209 & 0.241 & 0.13 \\
\hline & $\mathrm{f}$ & 163.60592 & -11.79784 & 0.6977 & & & & 22.885 & 4 & 5 & 685 & 0.787 & 0.13 \\
\hline & $\mathrm{g}^{*}$ & 163.62696 & -11.78158 & 0.6986 & & & & 19.654 & 2 & 3 & 718 & 0.825 & 0.36 \\
\hline & $\mathrm{h}$ & 163.62964 & -11.82359 & 0.6966 & & & & 22.199 & 1 & 2 & 1525 & 1.753 & -0.15 \\
\hline & $\mathrm{i}$ & 163.64143 & -11.82147 & 0.699 & & & & 21.098 & 2 & 2 & 1650 & 1.897 & 0.46 \\
\hline MACS J1149.5+2223 & $\mathrm{a}$ & 177.39015 & 22.40389 & 0.543 & & 23.162 & & 21.867 & 2 & 2 & 141 & 0.048 & -0.14 \\
\hline & $\mathrm{b}^{*}$ & 177.39855 & 22.38979 & 0.536 & & 23.149 & & 22.090 & 2 & 3 & 309 & 0.106 & -0.82 \\
\hline & $\mathrm{c}^{*}$ & 177.39863 & 22.39850 & 0.540 & & 19.258 & & 18.122 & 5 & 4 & 118 & 0.040 & -0.33 \\
\hline & d & 177.39977 & 22.39728 & 0.541 & & 21.197 & & 20.223 & 5 & 4 & 155 & 0.053 & -0.33 \\
\hline MACS J1206.2-0847 & $\mathrm{a}$ & 181.53955 & -8.81678 & 0.4356 & & 22.308 & & 21.427 & 2 & 2 & 409 & 0.202 & -0.73 \\
\hline & $b^{*}$ & 181.56187 & -8.80434 & 0.4265 & & 20.214 & & 19.616 & 3 & 3 & 241 & 0.119 & -2.24 \\
\hline & $\mathrm{c}$ & 181.57174 & -8.80643 & 0.4450 & & 20.156 & & 19.135 & 1 & 1 & 446 & 0.220 & 0.82 \\
\hline LCDCS 0541 & $\mathrm{a}$ & 188.09897 & -12.86970 & 0.5499 & & & & 21.022 & 2 & 3 & 867 & 1.008 & 2.80 \\
\hline & $\mathrm{b}$ & 188.10412 & -12.86525 & 0.5399 & & & & 20.615 & 2 & 3 & 712 & 0.827 & -0.50 \\
\hline & $\mathrm{c}$ & 188.11454 & -12.83518 & 0.5367 & & & & 19.587 & 4 & 1 & 328 & 0.382 & -1.56 \\
\hline & $\mathrm{d}$ & 188.12779 & -12.83268 & 0.5492 & & & & 21.795 & 2 & 2 & 249 & 0.289 & 2.57 \\
\hline & $\mathrm{e}$ & 188.13455 & -12.85739 & 0.5498 & & & & 20.053 & 1 & 2 & 372 & 0.432 & 2.76 \\
\hline & $\mathrm{f}$ & 188.14242 & -12.81575 & 0.5323 & & & & 22.953 & 1 & 2 & 734 & 0.854 & -3.02 \\
\hline & $\mathrm{g}$ & 188.16354 & -12.89634 & 0.5364 & & & & 21.641 & 2 & 3 & 1482 & 1.723 & -1.66 \\
\hline
\end{tabular}

Notes. Columns are: cluster name, galaxy identification, galaxy coordinates, redshift, magnitudes in the bands where images used for classification are available, jellyfish classes $F$ and $S$, projected distance to the cluster centre in kpc and in units of $r_{200}$, and ratio of the difference between the galaxy velocity and that of the cluster divided by the cluster velocity dispersion. 
Table 3. continued.

\begin{tabular}{|c|c|c|c|c|c|c|c|c|c|c|c|c|c|}
\hline Cluster & G. & RA & Dec & $z$ & $F 555 W$ & $F 606 W$ & $\begin{array}{l}F 702 W \\
F 775 W \\
\end{array}$ & $F 814 W$ & $F$ & $S$ & $\begin{array}{r}\text { Dist. } \\
(\mathrm{kpc}) \\
\end{array}$ & $\begin{array}{r}\text { Dist. } \\
\left(r_{200}\right)\end{array}$ & $\Delta v / \sigma_{\mathrm{v}}$ \\
\hline [MJM98]_034 & $a^{*}$ & 203.75482 & 37.82928 & 0.3841 & & & 23.213 & & 2 & 2 & 1303 & 1.300 & 0.22 \\
\hline LCDCS 0829 & $\mathrm{a}$ & 206.87102 & -11.76679 & 0.4534 & & 21.106 & & 20.397 & 4 & 4 & 320 & 0.196 & 0.29 \\
\hline \multirow[t]{7}{*}{ LCDCS 0853} & $a^{*}$ & 208.54225 & -12.51746 & 0.7565 & & & & 23.885 & 3 & 1 & 39 & 0.025 & -0.79 \\
\hline & $b^{*}$ & 208.54246 & -12.51466 & 0.7593 & & & & 21.036 & 2 & 3 & 78 & 0.049 & -0.43 \\
\hline & $\mathrm{c}$ & 208.55306 & -12.55668 & 0.7627 & & & & 22.491 & 2 & 2 & 1102 & 0.693 & 0.00 \\
\hline & $d^{*}$ & 208.55823 & -12.52258 & 0.7642 & & & & 21.076 & 1 & 3 & 485 & 0.305 & 0.19 \\
\hline & $\mathrm{e}$ & 208.56222 & -12.52241 & 0.7609 & & & & 21.117 & 3 & 2 & 586 & 0.368 & -0.23 \\
\hline & $\mathrm{f}$ & 208.57370 & -12.51199 & 0.7642 & & & & 24.061 & 2 & 1 & 884 & 0.556 & 0.19 \\
\hline & $\mathrm{g}$ & 208.57877 & -12.51216 & 0.7634 & & & & 21.426 & 2 & 3 & 1017 & 0.640 & 0.09 \\
\hline \multirow[t]{8}{*}{ 3C 295} & $\mathrm{a}$ & 212.79865 & 52.20013 & 0.454 & 23.593 & & & 21.908 & 1 & 3 & 1134 & 0.634 & -1.09 \\
\hline & $\mathrm{b}$ & 212.80549 & 52.16989 & 0.43 & & & & 22.916 & 1 & 2 & 1292 & 0.722 & -5.54 \\
\hline & $\mathrm{c}$ & 212.80823 & 52.19388 & 0.4485 & 22.968 & & & 21.459 & 2 & 4 & 977 & 0.546 & -2.10 \\
\hline & d & 212.82234 & 52.18692 & 0.44 & 23.455 & & & 22.452 & 2 & 2 & 791 & 0.442 & -3.67 \\
\hline & $\mathrm{e}$ & 212.82708 & 52.20480 & 0.4703 & 23.794 & & & & 4 & 2 & 530 & 0.296 & 1.86 \\
\hline & $\mathrm{f}$ & 212.83317 & 52.19815 & 0.4659 & 23.033 & & & & 3 & 3 & 468 & 0.261 & 1.07 \\
\hline & $\mathrm{g}$ & 212.83551 & 52.20273 & 0.464 & 22.0453 & & & & 3 & 4 & 377 & 0.211 & 0.72 \\
\hline & $\mathrm{h}$ & 212.85448 & 52.20978 & 0.47 & 25.132 & & & & 1 & 2 & 61 & 0.034 & 1.80 \\
\hline RX J1532.9+3021 & $\mathrm{a}$ & 233.22410 & 30.34982 & 0.3611 & & 17.819 & & 17.108 & 5 & 5 & 7 & 0.004 & 3.79 \\
\hline \multirow[t]{3}{*}{ MS $1621.5+2640$} & $\mathrm{a}$ & 245.89661 & 26.57446 & 0.4269 & 23.106 & & & 21.371 & 3 & 4 & 88 & 0.051 & 0.18 \\
\hline & $\mathrm{b}$ & 245.90057 & 26.57651 & 0.4405 & 22.796 & & & 21.103 & 3 & 3 & 136 & 0.079 & 2.84 \\
\hline & $\mathrm{c}$ & 245.92174 & 26.53319 & 0.4071 & & & & 21.700 & 2 & 5 & 885 & 0.515 & -3.78 \\
\hline RX J1716.4+6708 & $\mathrm{a}$ & 259.15717 & 67.12481 & 0.8044 & & & & 23.235 & 3 & 2 & 1421 & 0.843 & -0.94 \\
\hline \multirow[t]{2}{*}{ MACS J1931.8-2634 } & $\mathrm{a}$ & 292.94157 & -26.59913 & 0.3494 & & 20.890 & & 20.158 & 1 & 3 & 491 & 0.255 & -0.52 \\
\hline & $\mathrm{b}$ & 292.9506 & -26.57826 & 0.3652 & & 20.399 & & 20.131 & 3 & 4 & 115 & 0.060 & 2.61 \\
\hline MS 2053.7-0449 & $\mathrm{a}$ & 314.09464 & -4.59867 & 0.5880 & & 22.293 & & 21.687 & 2 & 2 & 715 & 0.441 & 0.81 \\
\hline \multirow[t]{5}{*}{ RX J2248.7-4431 } & $\mathrm{a}$ & 342.14918 & -44.52740 & 0.3356 & & 21.658 & & 21.043 & 1 & 2 & 569 & 0.247 & -2.01 \\
\hline & $\mathrm{b}$ & 342.15716 & -44.54514 & 0.3312 & & 23.971 & & 22.954 & 3 & 2 & 515 & 0.224 & -2.76 \\
\hline & $\mathrm{c}$ & 342.16731 & -44.51396 & 0.3517 & & 20.443 & & 19.991 & 5 & 5 & 362 & 0.157 & 0.70 \\
\hline & $d^{*}$ & 342.17550 & -44.53546 & 0.3362 & & 19.814 & & 19.106 & 4 & 4 & 155 & 0.067 & -1.91 \\
\hline & $\mathrm{e}$ & 342.20375 & -44.54226 & 0.3552 & & 21.698 & & 21.293 & 2 & 2 & 464 & 0.202 & 1.29 \\
\hline
\end{tabular}

$G \times M_{200}=100 \times H(z)^{2} \times r_{200}^{3}$,

where

$H(z)=H_{0} \times\left[\Omega_{m}(1+z)^{3}+\Omega_{\Lambda}\right]^{(1 / 2)}$

is the Hubble parameter at the cluster redshift, $z$, computed with the cosmological parameters given at the end of Sect. 1, and $G$ is the gravitational constant. We also compute the cluster velocity dispersion using Eq. (1) from Munari et al. (2013):

$\sigma_{\mathrm{v}}=1090 \times\left[h(z) \times M_{200}\right]^{(1 / 3)}$,

where $h(z)=H(z) / 100, M_{200}$ is expressed in units of $10^{15} M_{\odot}$, and $\sigma_{\mathrm{v}}$ is the unidimensional velocity dispersion in units of $\mathrm{km} / \mathrm{s}$. The values of $r_{200}$ and $\sigma_{\mathrm{v}}$ are given in Table 1 for all clusters that have jellyfish candidates.

For each galaxy, we compute its velocity relative to the mean cluster velocity in units of $\sigma_{\mathrm{v}}$, and give the corresponding values in Tables 2 and 3.

\section{Jellyfish candidates in MACS J0717.5+3745 $(z=0.5458)$}

\subsection{The catalogue of jellyfish candidates in MACS J0717.5+3745}

MACS0717 is a well-known massive cluster with a large extension/filament reaching a total of about $9 \mathrm{Mpc}$ towards the southeast and studied by Jauzac et al. (2012, 2018a,b) and Martinet et al. (2016). Based on a weak lensing study, its mass was estimated to be $2.4 \times 10^{15} M_{\odot}$ within the $R_{200}$ radius

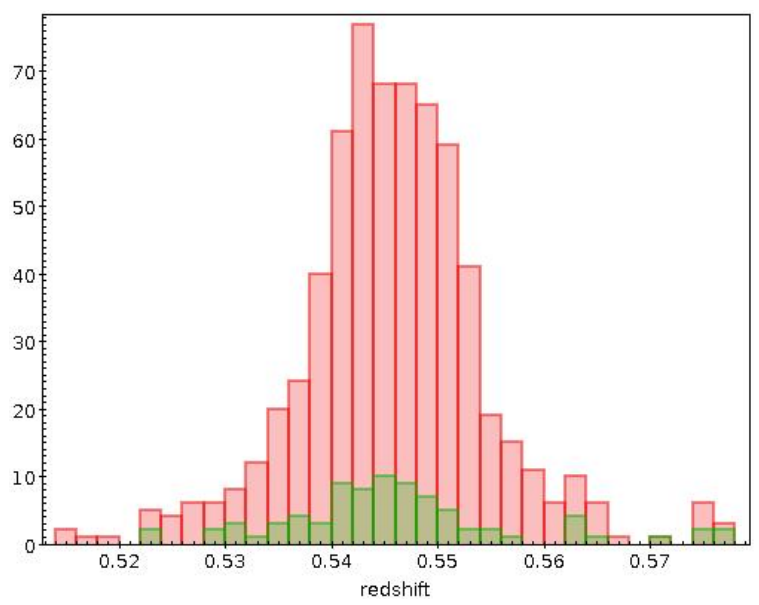

Fig. 1. Redshift histogram of the 646 galaxies in all the MACS0717 region (in red), and redshift histogram of the 81 candidate jellyfish galaxies (in green).

by Martinet et al. (2016). An extensive spectroscopic redshift catalogue, with 646 galaxies in the redshift interval $0.5145 \leq$ $z \leq 0.5785$, allows us to search for jellyfish galaxies in a very efficient way throughout the structure, which has been almost entirely covered by HST/ACS observations.

For this cluster, we examined 18 individual HST/ACS images in both $F 606 \mathrm{~W}$ and $F 814 \mathrm{~W}$ bands. Out of the 646 galaxies with redshifts in the cluster range, 81 were identified as jellyfish candidates. The list of these galaxies is given in Table 2, and corresponding images are displayed in Appendix A. 


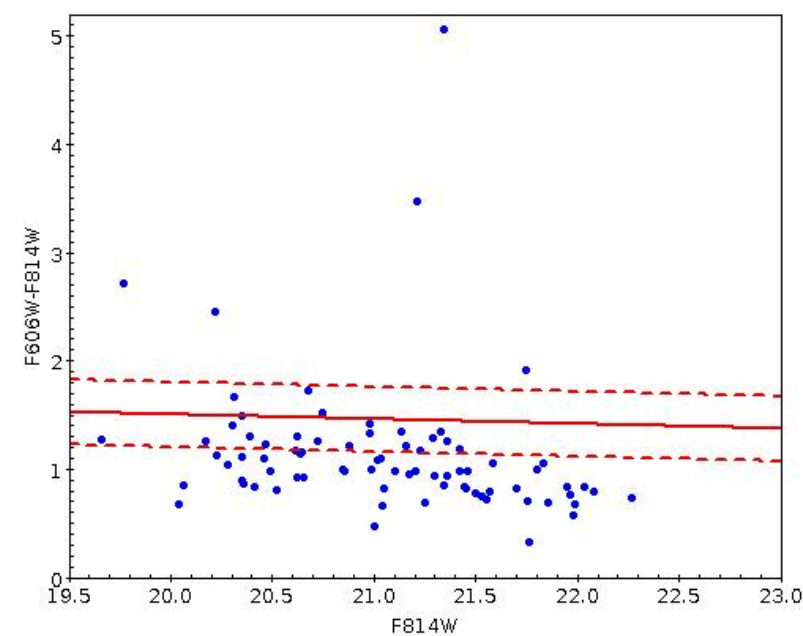

Fig. 2. Colour-magnitude diagram for MACS0717. The blue points show the 81 candidate jellyfish galaxies belonging to the cluster. The solid red line shows the position of the red sequence and the dashed lines correspond to \pm 0.3 on either side of the red sequence.

The histograms of all redshifts available in the MACS0717 region and of the identified jellyfish galaxy candidates is shown in Fig. 1. This plot seems to show that jellyfish candidates generally follow the velocity distribution of the cluster.

The jellyfish classification was separately made by two of us, and the corresponding classes are given in the last two columns (classS and classF) of Table 2. It appears that classF is often stricter than classS, so for some results shown below we made two samples of strong probability jellyfish objects (i.e. of types 3, 4, and 5), according to classF and to classS separately.

The colour-magnitude diagram for MACS0717 is shown in Fig. 2. The red sequence drawn from Subaru data in the $V$ and $I$ bands was $V-I=-0.0436 \times I+2.75$ (Durret et al. 2016), computed by considering the positions on this sequence of several tens of galaxies at the cluster redshift. Its width of \pm 0.3 was chosen to include all the galaxies belonging to the cluster according to their spectroscopic redshift, as explained by Durret et al. (2016). We adapted this red sequence to the $F 606 \mathrm{~W}$ and $F 814 W$ filters using the transformations given by Fukugita et al. (1995) and the result is shown in Fig. 2 (red lines). The data points refer to the jellyfish candidates found in the cluster. We can see that most of them are blue and lie below the red sequence. For the four galaxies located notably above the red sequence, the fit of the SED by a stellar population model (see Sect. 5.2.1) gives internal extinctions of 0.4 for three galaxies, and 0 for the fourth one. Thus, except for the last galaxy, their red colours may be at least partly explained by internal dust.

\subsection{Spatial distribution of jellyfish candidates in MACS J0717.5+3745}

The positions of the jellyfish candidates in MACS0717 are shown in Fig. 3. We highlight galaxies of types 3, 4, and 5 (considering both classifications) since they have a high probability of being real jellyfish galaxies. We can see that a large number is located outside the densest regions and about one third lie in regions less dense than the $3 \sigma$ contour above the average background density. Interestingly, about half of the jelly- fish candidates are located in the southern extended filament region of the cluster (filament C in Durret et al. (2016), the vertical yellow ellipse in Fig. 3), a low-density zone where only faint X-ray emission is detected (Ma et al. 2009), and therefore where RPS is not expected to be strong but might be gently acting.

Another way to illustrate the spatial distribution of jellyfish galaxies in the large-scale MACS0717 structure is to draw the histogram of projected distances to the cluster centre, as shown in Fig. 4. This figure confirms the paucity of jellyfish galaxies in the innermost cluster regions. The histogram of the galaxy velocities in units of $\sigma_{\mathrm{v}}$ has a similar shape. This lack of jellyfish candidates in the cluster centre agrees with the model by Safarzadeh \& Loeb (2019) that predicts no jellyfish galaxies at small clustercentric distances. Based on detailed hydrodynamical simulations, Yun et al. (2019) also found jellyfish galaxies to be more frequent at intermediate and large cluster-centric 3D distances. This result is at odds with the results of the GASP survey, where jellyfish galaxies at low redshift tend to be found in the innermost regions of clusters (Gullieuszik et al. 2020).

A morphometric analysis of the jellyfish galaxies in MACS0717, comparable to that performed by Roman-Oliveira et al. (2021) with MORFOMETRYKA on a large sample of rampressure stripping candidates in the Abell 901/902 multi-cluster system, would be very interesting.

\section{Jellyfish candidates in 22 other clusters}

\subsection{The jellyfish catalogue}

Positions and magnitudes of the 97 jellyfish candidates found in 22 clusters are given in Table 3. For CLASH clusters, galaxy coordinates are those of the CLASH catalogue, which always match very well those measured in the images.

For the DAFT/FADA clusters, galaxy coordinates are those measured by SExtractor on the HST images, as they are more accurate than some of the coordinates extracted from NED. We checked by superimposing galaxies from the SDSS catalogue that the astrometry of our HST images was correct.

The redshift histogram of the 97 jellyfish candidates found in 22 clusters (other than MACS0717) is shown in Fig. 5.

\subsection{Images and notes on individual objects}

The images of the 97 jellyfish candidates are shown in Appendix B. In some cases, we give below a few indications on specific galaxies when we think it is useful and we indicate if clusters are merging whenever this information is available. In particular, we mention if the positions of the jellyfish candidates lie in the direction of the general elongation of the cluster, defined both from the position angle of the brightest cluster galaxy, from the alignment of the brightest cluster galaxies, and from the red-sequence galaxy density maps drawn by Durret et al. $(2016,2019)$ when available. This direction should trace the orientation of the filamentary regions, at large scale, where each cluster is embedded, and along which one would expect the largest galaxy infall to happen (e.g. West 1994; West et al. 2017). Thus, these regions are the privileged areas for infalling late-type galaxies to become jellyfish as they enter with high speed and move across the cluster denser environment.

When two galaxies are located in the same frame, the galaxy with a spectroscopic redshift at the cluster redshift is indicated with a circle in images of Appendix B. 


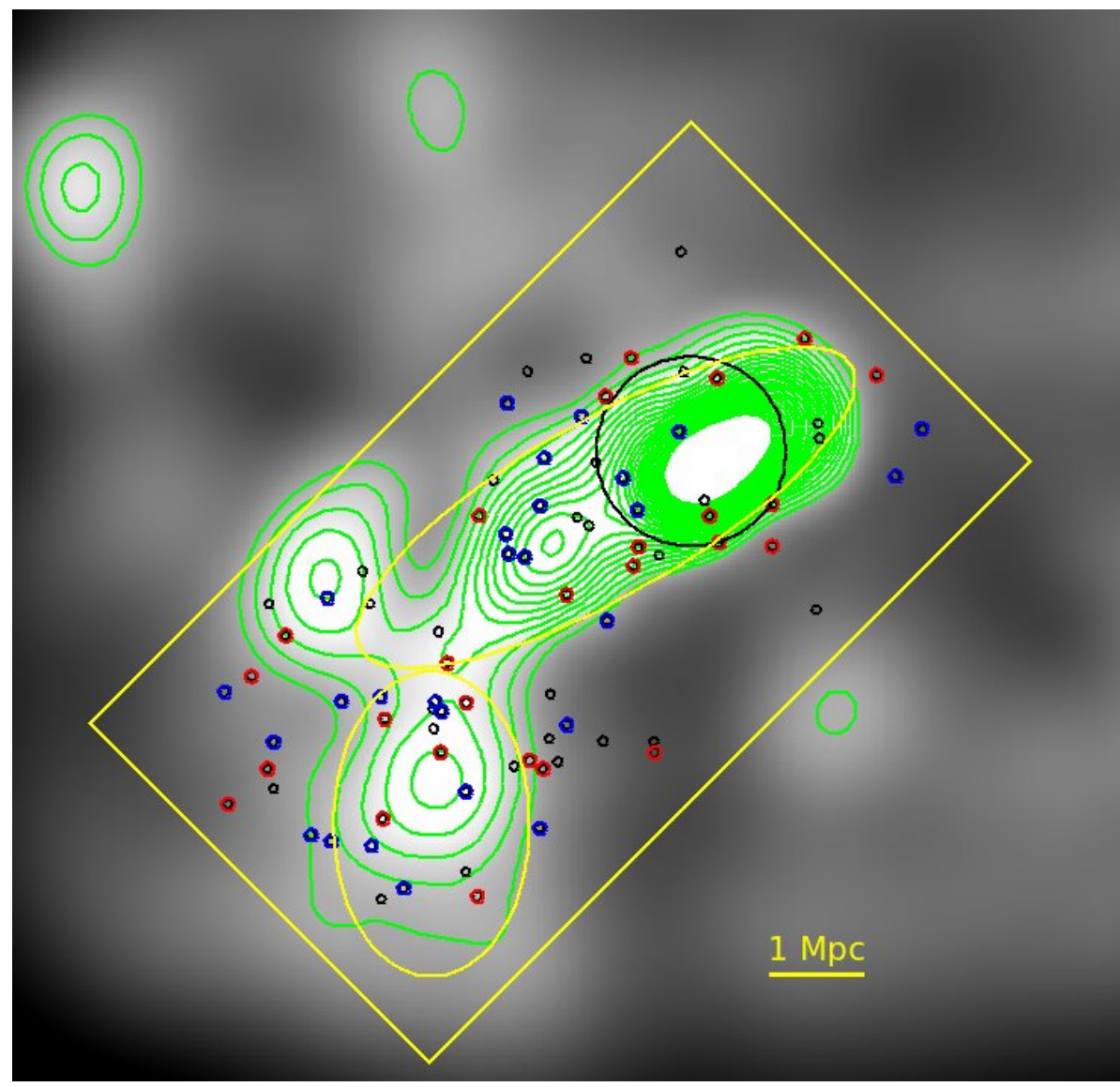

Fig. 3. Density map of red sequence galaxies shown with grey shading and green isocontours, taken from Durret et al. (2016). The black circle is centred on the cluster centre, and has a $1 \mathrm{Mpc}$ radius. The two yellow ellipses show the $3 \sigma$ contours of the density distribution. The positions of the candidate jellyfish galaxies are indicated as follows: red circles indicate galaxies classified as jellyfish types 3, 4 , and 5 according to the strictest classification; blue circles indicate additional galaxies classified as types 3, 4, and 5 according to the less strict classification; black circles indicate all candidate jellyfish galaxies from Table 2 . The yellow rectangle shows the approximate HST coverage.

\subsection{1. $\mathrm{Cl} 0016+16(z=0.5455)$}

Images of the eight jellyfish galaxy candidates in $\mathrm{Cl0016}+16$ are shown in Fig. B.1. Out of the eight jellyfish galaxies (out of 103 galaxies at the cluster redshift), four are well aligned with the general cluster elongation (see Durret et al. 2019, Fig. B.1), and three others are not far from this region/direction.

\subsubsection{Abell $209(z=0.206)$}

Images of the two jellyfish galaxy candidates (out of 39 galaxies at the cluster redshift) are shown in Fig. B.2. One of them is aligned along the cluster main elongation region (Durret et al. 2019, Fig. B.2).

\subsection{3. $\mathrm{Cl} 0152.7-1357(z=0.831)$}

Images of the six jellyfish galaxy candidates (out of 66 galaxies at the cluster redshift) are shown in Fig. B.3. All of them are in the northern part of this merging cluster (see Guennou et al. 2014 and references therein), which is the main zone covered by the HST image. Based on X-ray data and on a large number of galaxy spectroscopic redshifts, Girardi et al. (2005) showed that C10152.7-1357 consists of three galaxy clumps of different mean velocities: a low velocity clump in the central-south-west region, a high velocity clump in the eastern region and a weaker eastern clump.

\subsubsection{Abell $383(z=0.1871)$}

Images of the two jellyfish galaxy candidates (out of 32 galaxies at the cluster redshift) are shown in Fig. B.4. One is located in the cluster elongation area (Durret et al. 2019, Fig. B.5), one is close to this region, and the third one is further out.

\subsubsection{MACS J0416.1-2403 $(z=0.396)$}

Images of the eight jellyfish galaxy candidates (out of 205 galaxies at the cluster redshift) are shown in Fig. B.5. Galaxy f may be superimposed on a gravitational arc. Out of the eight jellyfish galaxies, seven seem to be roughly spatially aligned with the main cluster merging axis.

\subsubsection{MACS J0429.6-0253 $(z=0.399)$}

Images of the two jellyfish galaxy candidates (out of only two galaxies at the cluster redshift) are shown in Fig. B.6. None are located along the main cluster elongation area.

\subsubsection{MACS J0454.1-0300 $(z=0.5377)$}

Images of the five jellyfish galaxy candidates (out of 92 galaxies at the cluster redshift) are shown in Fig. B.7. Galaxy a may not be a jellyfish galaxy, but we keep it in the sample because of its two nuclei. Two galaxies follow the main cluster elongation, and a third one is close-by.

\subsubsection{Abell $851(z=0.4069)$}

Images of the 11 jellyfish galaxy candidates (out of 102 galaxies at the cluster redshift) are shown in Fig. B.8. All but one are located in the northern half of the cluster, but with no specific alignment. This is a merging cluster (Durret et al. 2016). 

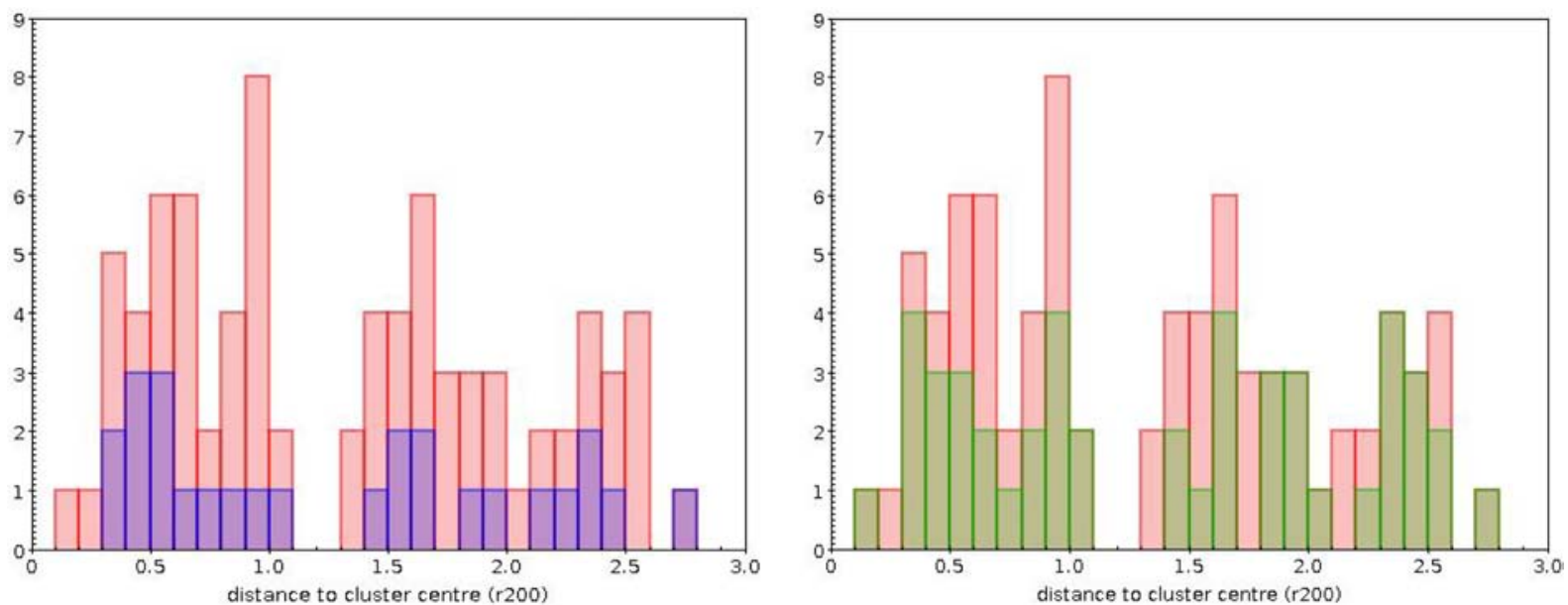

Fig. 4. Histogram (in red) of the projected distance to the cluster centre of in MACS0717 (81 galaxies). Left: candidate jellyfish galaxies (superimposed in blue) of classes 3, 4, and 5 according to the strictest classification (26 galaxies). Right: candidate jellyfish galaxies (superimposed in green) of classes 3, 4, and 5 according to the less strict classification (47 galaxies). Distances are in in units of $r_{200}$.

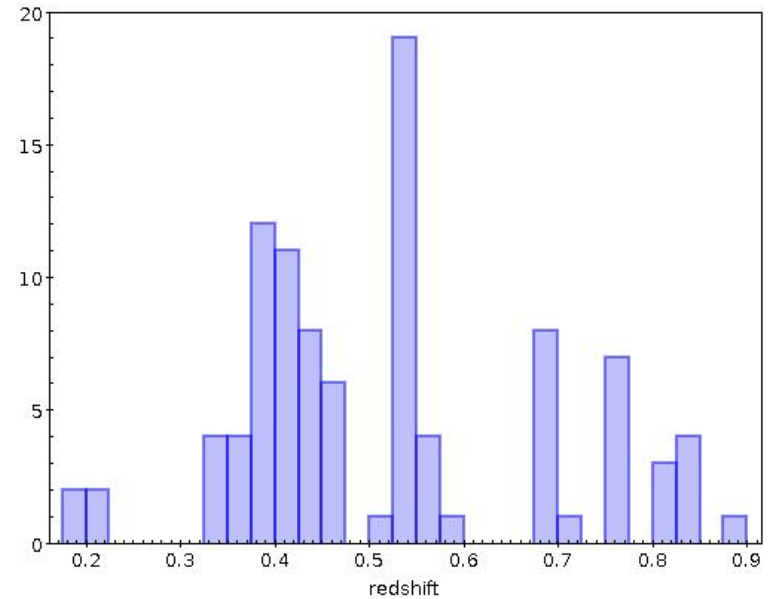

Fig. 5. Redshift histogram of the 97 candidate jellyfish galaxies in 22 clusters (excluding MACS0717).

\subsection{9. $\operatorname{LCDCS} 0172(z=0.6972)$}

The images of the nine candidate jellyfish galaxies (out of 45 galaxies at the cluster redshift) are displayed in Fig. B.9. They show no particular spatial distribution.

\subsubsection{MACS J1149.5+2223 $(z=0.544)$}

Images of the four jellyfish galaxy candidates (out of 106 galaxies at the cluster redshift) are shown in Fig. B.10. Three of them follow the main cluster elongation. Galaxies $\mathrm{c}$ and $\mathrm{d}$ are interacting, with many filaments in their neighbourhood. Thus the image also shows their environment.

\subsubsection{MACS J1206.2-0847 $(z=0.44)$}

Images of the three jellyfish galaxy candidates (out of 64 galaxies at the cluster redshift) are shown in Fig. B.11. Two of these galaxies are located along the main cluster elongation.

\subsubsection{2. $\operatorname{LCDCS} 0541(z=0.5414)$}

Images of the seven jellyfish galaxy candidates (out of 80 galaxies at the cluster redshift) are shown in Fig. B.12. Their spatial distribution shows no particular trend. The bright arc north and east of galaxy e is a gravitational arc.

\subsubsection{3. [MJM98]_034 $(z=0.383)$}

Image of the single jellyfish galaxy candidate (out of eight galaxies at the cluster redshift) is shown in Fig. B.13. As noted by Guennou et al. (2014) this cluster is at redshift $z=0.383$ and not at $z=0.5950$ as found in NED.

\subsubsection{LCDCS $0829=\mathrm{RXJ1347}(z=0.451)$}

Image of the single jellyfish galaxy candidate (out of 35 galaxies at the cluster redshift) is shown in Fig. B.14. Its position is roughly aligned with the cluster elongation.

\subsubsection{LCDCS $0853(z=0.7627)$}

Images of the seven jellyfish galaxy candidates (out of 20 galaxies at the cluster redshift) are shown in Fig. B.15. All seven galaxies are located in the south-east quarter of the cluster.

\subsubsection{C $295(z=0.4600)$}

Images of the eight jellyfish galaxy candidates (out of 30 galaxies at the cluster redshift) are shown in Fig. B.16. They show no particular distribution throughout the cluster. Due to different spatial coverage, some appear in both filters, others only in one.

\subsubsection{RX J1532.9+3021 $(z=0.345)$}

Image of the single jellyfish galaxy candidate (this galaxy is in fact the brightest cluster galaxy, and there is only one other galaxy with a measured redshift in the cluster) is shown in Fig. B.17 A zoomed-in image showing a disturbed structure is also shown.

\subsubsection{MS $1621.5+2640(z=0.426)$}

Images of the three jellyfish galaxy candidates (out of 31 galaxies at the cluster redshift) are shown in Fig. B.18. They show no particular distribution in the cluster. The two filters do not cover exactly the same region. Galaxy a may be interacting with one or two galaxies, but spectroscopic redshifts are not available for these objects. Galaxy $b$ has a plume of emission to the south, 

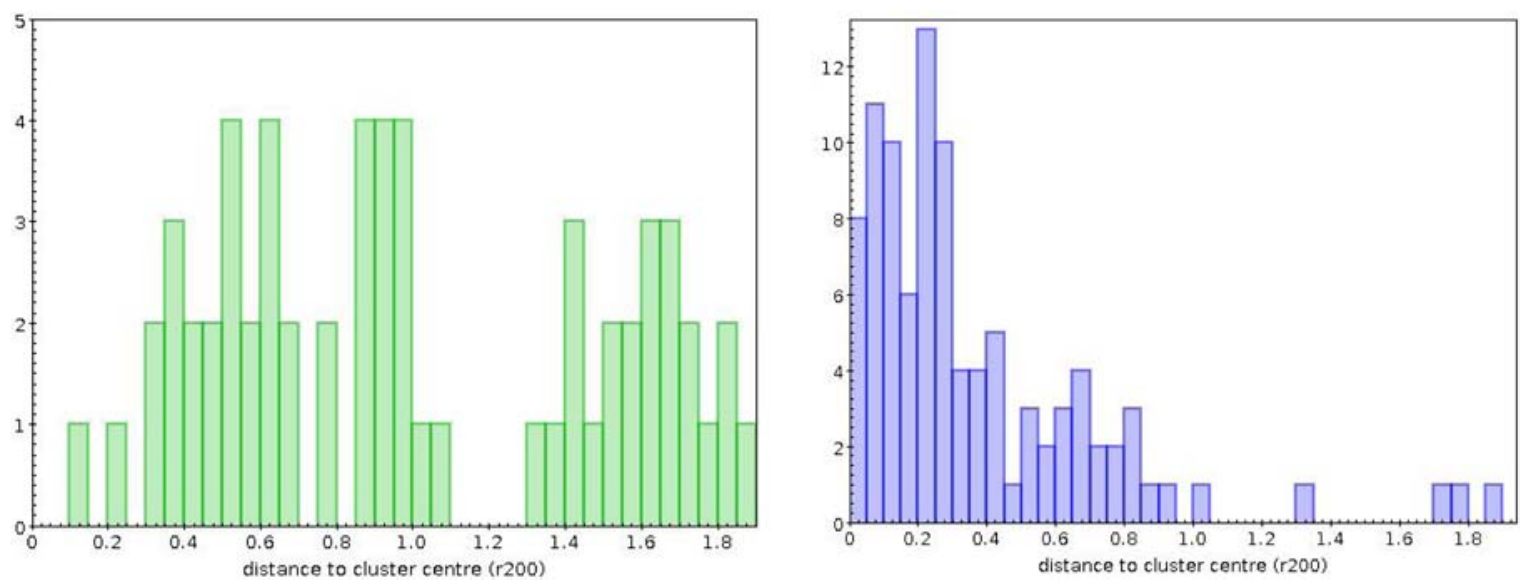

Fig. 6. Left: histogram of the projected distance of 61 jellyfish galaxy candidates to the cluster centre for MACS0717, in units of $r_{200}$, with the same limit as the figure on the right. Right: same histogram for the 97 jellyfish galaxy candidates of the other clusters.

and seems to be surrounded by gravitational arcs in the north. Spectroscopy is also needed to confirm the jet-like feature east of galaxy c.

\subsubsection{9. $\mathrm{RX} \mathrm{J1716.4+6708}(z=0.813)$}

Image of the single jellyfish galaxy candidate (out of 22 galaxies at the cluster redshift) is shown in Fig. B.19.

\subsubsection{MACS J1931.8-2634 ( $z=0.352)$}

Images of the two jellyfish galaxy candidates (out of only three galaxies at the cluster redshift) are shown in Fig. B.20.

\subsubsection{MS 2053.7-0449 $(z=0.583)$}

Image of the single jellyfish galaxy candidate (out of 32 galaxies at the cluster redshift) is shown in Fig. B.21. It lies exactly north of the cluster centre, positioned along the direction of the cluster elongation.

\subsubsection{2. $\mathrm{RX} \mathrm{J} 2248.7-4431(z=0.348)$}

Images of the five jellyfish galaxy candidates (out of 42 galaxies at the cluster redshift) are shown in Fig. B.22. Two out of five galaxies are located within the cluster elongation area/direction.

\section{Jellyfish galaxy candidate properties}

\subsection{Spatial distribution relative to the cluster centre}

The histogram of the projected distances of jellyfish galaxy candidates to the centre of the respective cluster is shown in Fig. 6, where we compare the result already shown for MAC0717 but limited to within $1800 \mathrm{kpc}$ (on the left) with the results obtained for all the other clusters taken together (right panel). This figure shows that the number of jellyfish galaxy candidates in the central region of MACS0717 is rather small, whereas it is large in the ensemble of all the other clusters. Unlike for MACS0717, our coverage of the remaining clusters is far from complete; nonetheless, this result indicates that in MACS0717 there seems to be a real absence of jellyfish galaxy candidates in the innermost cluster region.

\subsection{Spectral energy distribution}

\subsubsection{SED fitting for jellyfish galaxy candidates in MACS J0717.4+3745}

We matched our catalogue of 81 jellyfish galaxy candidates detected in MACS0717 with the eight band optical and infrared ground-based catalogue covering the entire region of MACS0717 described in Sect. 2. We found 79 galaxies in common using a match radius of 1.5 arcsec.

We then used LePhare (Ilbert et al. 2006), through the GAZPAR interface ${ }^{7}$, to fit the SEDs of these 79 jellyfish galaxy candidates with the Bruzual \& Charlot (2003) models and the Chabrier (2003) initial mass function: Based on an input catalogue with positions, magnitudes and corresponding errors, and in our case spectroscopic redshift. LePhare fits the galaxy SED, computes absolute magnitudes in the input bands, and infers, from the best fit template in each case, the stellar mass, star formation rate, specific star formation rate (sSFR), mean stellar population age, as well as other quantities that we will not consider here. The input parameter space was carefully selected so as to cover the expected characteristics of late-type galaxies with probable SF activity.

We can note that for all but a few of the 79 galaxies, the best fit template spectrum includes an $\mathrm{H} \alpha$ line, and among these about $80 \%$ of the template spectra include all the main emission lines in the optical ([OII]3727, [OIII]4959, 5007, $\mathrm{H} \beta$ and $\mathrm{H} \alpha$ ), thus implying that the majority of our jellyfish candidates are forming stars. As an illustration of the obtained fits, we show in Fig. 7 the SED and best fit templates for two galaxies, one with only a weak $\mathrm{H} \alpha$ emission line and one with several emission lines in the best fit template. We see that these fits are quite good, and this was indeed the case for all galaxies that GAZPAR/LePhare analysed.

The histogram of jellyfish galaxy candidate stellar masses in MACS0717 is shown in the left-hand panel of Fig. 8. The galaxies cover the range of stellar masses between $10^{9}$ and $10^{11} M_{\odot}$. We divided the sample into high-mass $(\log M \geq 10)$ and lowmass $(\log M<10)$ galaxies and checked their spatial distribution but found no difference between these two samples.

The histogram of jellyfish galaxy candidate SFRs for MACS0717 is shown in the left hand panel of Fig. 9. The SFRs cover a large range, essentially between 0.1 and $60 M_{\odot} \mathrm{yr}^{-1}$. We also show in Fig. 10 the histogram of the sSFRs in MACS0717:

\footnotetext{
7 https://gazpar.lam. fr/home
} 

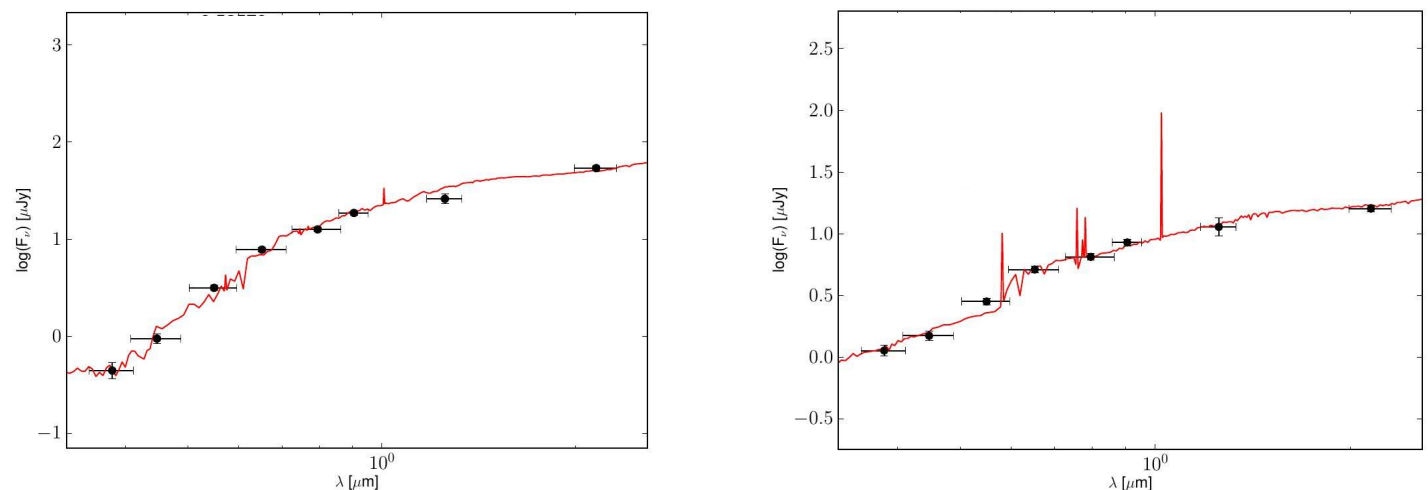

Fig. 7. Two jellyfish galaxy candidates in MACS0717 (in black: 8 mag available from the Jauzac et al. 2012 catalogue) with the best stellar population fit superimposed (in red). One has a weak $\mathrm{H} \alpha$ emission line (left) and one has several strong emission lines (right).
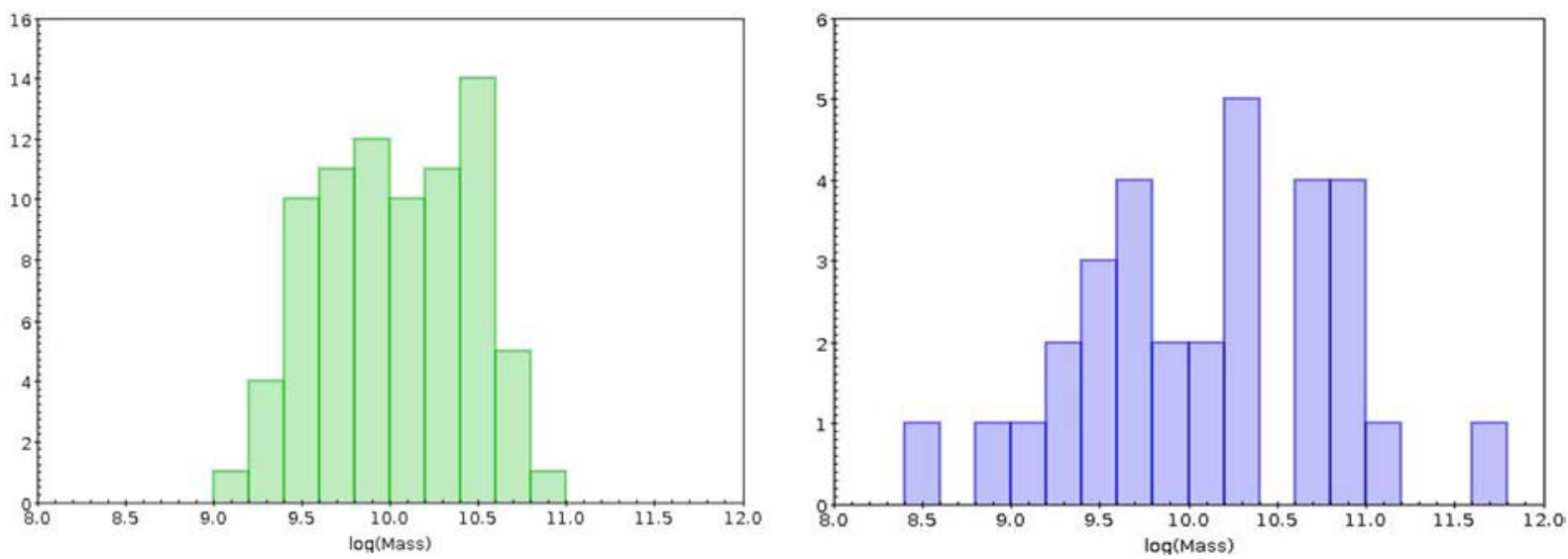

Fig. 8. Histograms of the stellar masses for the 79 jellyfish galaxy candidates in MACS0717 (left), and 31 galaxies of other clusters (right).
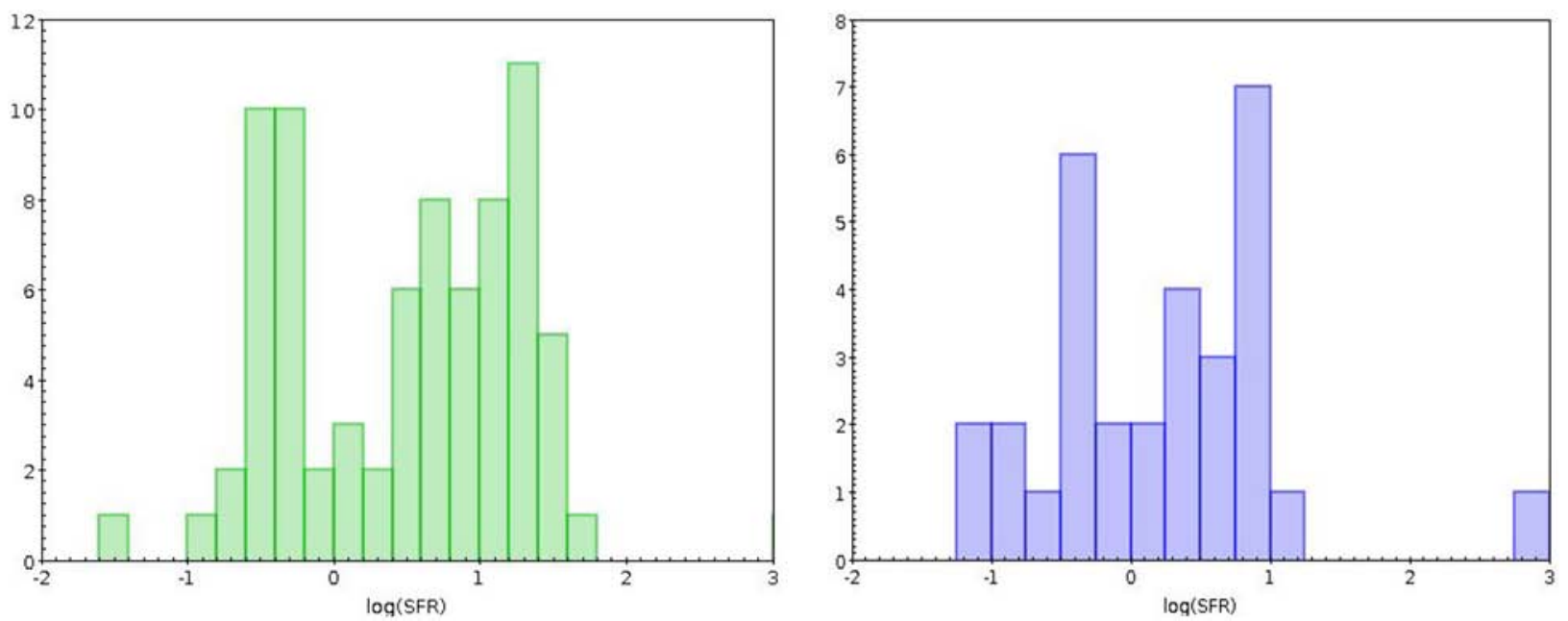

Fig. 9. Histograms of the SFRs for the 79 jellyfish galaxy candidates in MACS0717 (left), and 31 galaxies of other clusters (right).

Values go from $10^{-12}$ to $10^{-8} \mathrm{yr}^{-1}$ (except for two galaxies that have very low sSFRs and do not appear on the figure), and more than 30 galaxies have sSFR $>10^{-9} \mathrm{yr}^{-1}$. We mark on the sSFR histograms the indicative value of $\log (\mathrm{sSFR})=-11$ below which galaxies are commonly considered to be quiescent.

The histogram of the stellar population age for the jellyfish galaxy candidates in MACS0717 is shown in Fig. 11. We can see that more than half of the galaxies are, on average, younger than $1.5 \times 10^{9} \mathrm{yrs}$, so the stellar population is globally quite young.
The relation between the SFR and galaxy stellar masses is shown in Fig. 12. We superimposed on this plot the main sequence of SF galaxies as determined by Peng et al. (2010), based on a very large sample of galaxies from the SDSS and zCOSMOS surveys, and its dispersion that we estimated to be \pm 0.5 around the relation (see their Fig. 1). We see that $12(15 \%)$ jellyfish galaxies are below this sequence, $23(29 \%)$ are in the interval of Peng et al. (2010), and the 44 other ones (56\%) lie above the SF main sequence. 

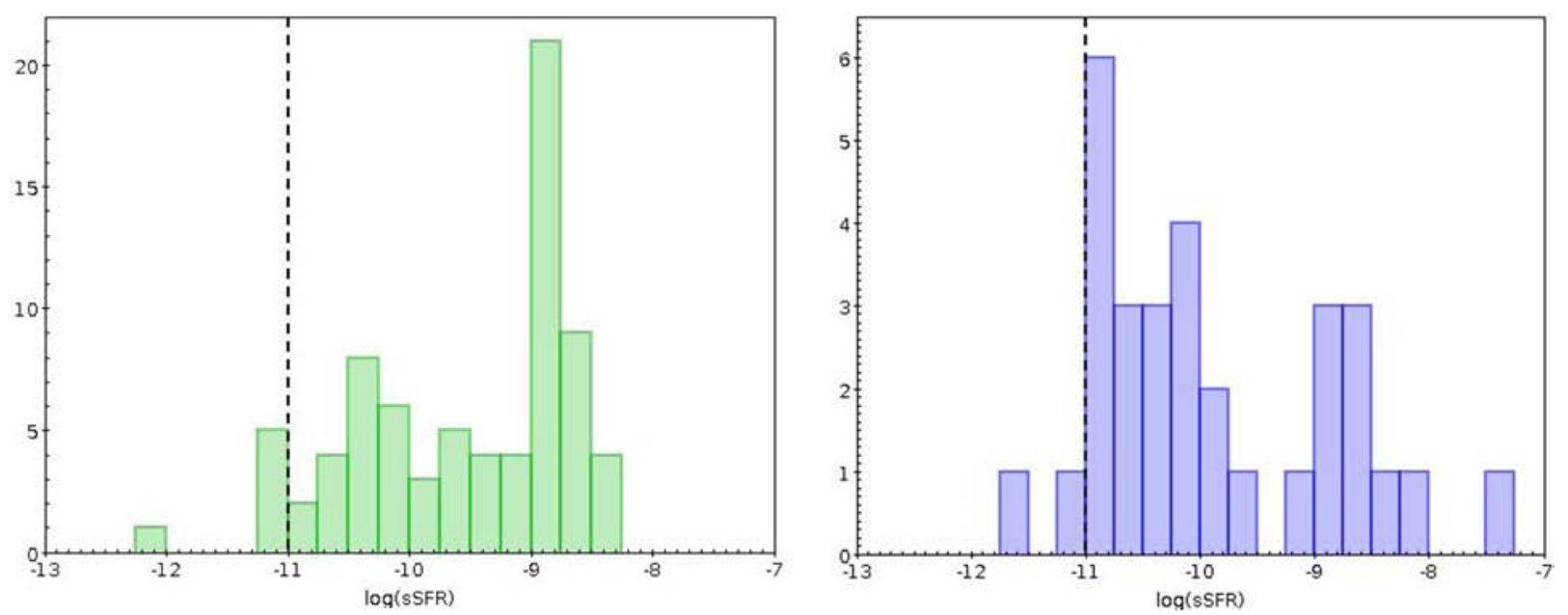

Fig. 10. Histograms of the sSFRs of the 79 jellyfish galaxy candidates in MACS0717 (left), and 31 galaxies in the other clusters (right). The black vertical dashed line shows the value of -11 below which galaxies are considered as quiescent.
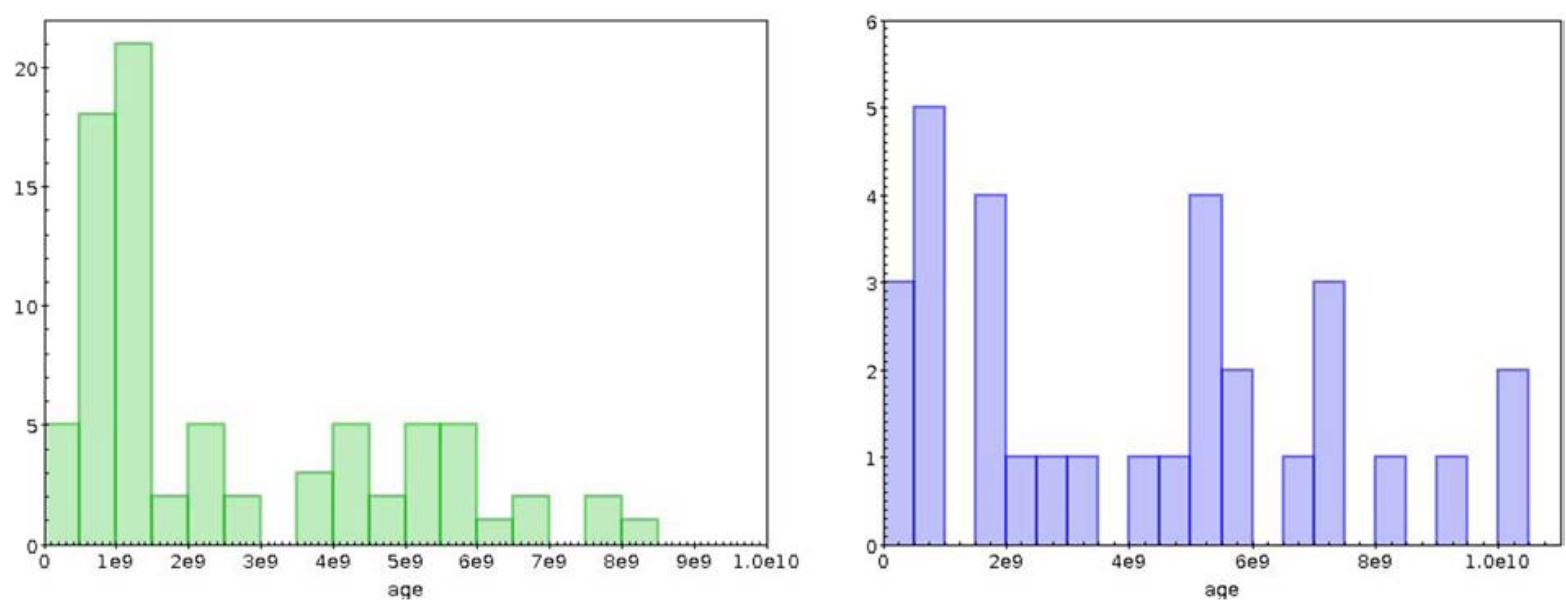

Fig. 11. Histograms of the stellar population ages of the 79 jellyfish galaxy candidates in MACS0717 (left), and 31 galaxies in the other clusters (right).

In agreement with the previously noted fact that SEDs of the jellyfish galaxies in MACS0717 are in majority best fitted by a spectrum including one or several emission lines, this confirms that, in average, jellyfish galaxy candidates seem to have a higher SFR than "normal" star-forming galaxies of the same stellar mass. Figure 12 suggests that the majority seems to form a sequence parallel to that of Peng et al. (2010), but with an SFR about ten times higher. There are, however, a few cases of very low specific star formation rates: These galaxies are apparently quenched, as indicated by the $\log (\mathrm{sSFR})<-11$ criterion (see Fig. 10).

As a comparison, we also obtain an SED fit for 442 galaxies in the same redshift interval but not classified as jellyfish candidates. Out of these, 113 galaxies can be considered as nonquiescent (specific star formation rate $\log (\mathrm{sSFR}) \geq-11)$. We also include these galaxies in Fig. 12. One can see that they cover more or less the same region as the jellyfish candidates. However, if we calculate the average sSFR for the jellyfish candidates and for this control sample, both limited to $\log$ SSFR $\geq-11$ (respectively: 71 and 113 galaxies), we find respective values of -9.36 and -9.87 , suggesting that the sSFR is about 3 times larger for jellyfish candidates. In view of the errors on these quantities, this value of 3 should not be taken at face value, but it does suggest that jellyfish galaxies have an sSFR higher than that of normal galaxies.

We also overplot on Fig. 12 (left panel) the relations found by Vulcani et al. (2018) for disks of ram pressure stripped galaxies (in blue), and undisturbed galaxies (in black). One can note that at least half of our jellyfish candidates are located above both their sequences.

As a final check, and since jellyfish galaxies are very often systems with an increased SFR (see the Introduction), we verified if our results were affected by classification errors in the following manner. We divided our sample in two sub-samples based on the classF column of Table 2: types 1 and 2 on one side, types 3, 4 and 5 on the other. We then checked the numbers of each type in Fig. 12 (left), to see if the galaxies with an SFR lower than the main sequence were in majority of types 1 or 2 (i.e. galaxies that may not be jellyfish galaxies after all). The percentages of jellyfish galaxy candidates of types 1 and 2 with an SFR below, in, and above the Peng sequence are comparable to those given above for all the jellyfish galaxy candidates. We therefore consider that galaxies with a low SFR are not necessarily doubtful jellyfish galaxies. This means that a number of our jellyfish candidates are indeed undergoing a phase of low SF activity. 

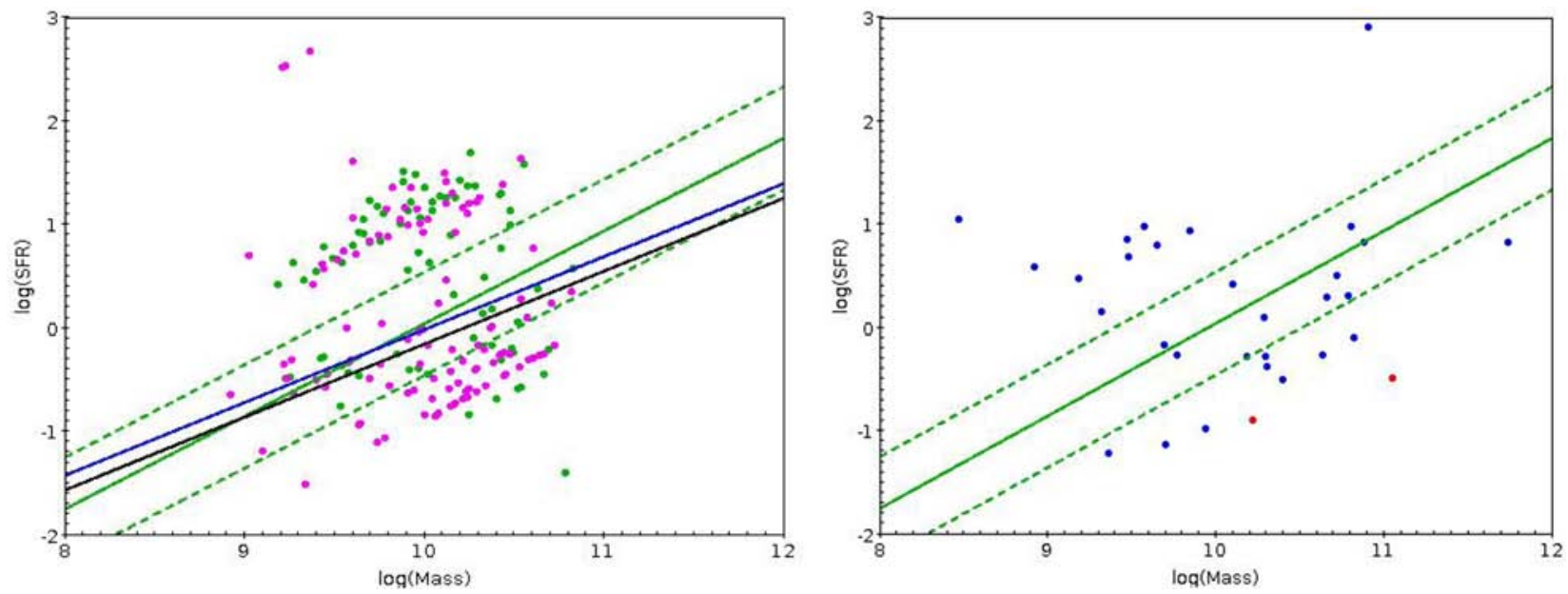

Fig. 12. SFR as a function of stellar mass for the 79 jellyfish galaxy candidates in MACS0717 (left) and 31 galaxies of other clusters (right). On both figures, the three green lines indicate the relation found by Peng et al. (2010) and its approximate dispersion of \pm 0.5 (dashed lines). On the left figure, pink dots correspond to normal cluster galaxies with $\log (\mathrm{SSFR}) \geq-11$ (see text). Blue and black lines show the relations found by Vulcani et al. (2018) for the disk SFR-mass relation for stripping and control sample galaxies respectively (see their Fig. 1). On the right panel, the two red points highlight the two galaxies with $\log (\mathrm{sSFR})<-11$.

\subsubsection{AGN activity in MACS0717 jellyfish candidates}

An interesting link between the presence of jellyfish features and AGN activity was explored by Poggianti et al. (2017b), who found a strong correlation between these parameters for the most extreme examples of jellyfish galaxies in their GASP sample. Motivated by this, we checked if any of our jellyfish candidates in MACS0717 showed signs of hosting an AGN. This is a hard task with the limited data available so, considering the possibilities, we opted for using the MIR criteria, based on WISE colours, developed by Mateos et al. (2012) and Stern et al. (2012). Both are optimized to select luminous AGN so we will likely just uncover the tip of the iceberg.

We used the Table Access Protocol (TAP) Query service of TOPCAT (Taylor 2005) to access and download the DR8 tractor catalogue limited to the area covered by the HST observations. This catalogue contains magnitudes in all four WISE bands, extracted by the DESI team in preparation for their Legacy Survey, that go about 1 magnitude deeper than the original AllWISE ones (D. Schlegel, priv. comm.). We converted these deredenned $\mathrm{AB}$ magnitudes to the Vega system, following the DESI webpage information ${ }^{8}$, to apply directly the above mentioned criteria. We then matched this catalogue with our own: For a search radius of $1.5 \operatorname{arcsec}, 79$ of our 81 galaxies had data in the DR8 tractor catalogue, allowing us to check their $W 1-W 2$ colour index. Out of these, only two barely pass the Stern et al. (2012) threshold for identifying AGN, i.e. a colour index $W 1-W 2>0.8$, imposing as well an $S / N>5$ for the WISE individual magnitudes. These are galaxies \#9 and \#59 of Table 2. Applying the stricter Mateos et al. (2012) criterion, in the $W 1-W 2$ versus $W 2-W 3$ colour plane, results in no AGN candidates.

Our marginal AGN candidates are located in regions of high density. One lies on top of the $1 \mathrm{Mpc}$ central radius (black circle in Fig. 3), to the right, has $v / \sigma_{\mathrm{v}} \sim-1, W 1-W 2=0.90$ and $\mathrm{J}$ class 4 attributed by both FD and SC: It is galaxy \#9 of Table 2, marked with a red symbol in Fig. 3. The other one lies close to the northern edge of the yellow vertical ellipse of Fig. 3, where it is marked by a blue symbol (galaxy \#63; J class

\footnotetext{
8 https://www.legacysurvey.org/dr8/description/
}

2 and 3 by FD and SC, respectively) and has $v / \sigma_{\mathrm{v}} \sim-0.5$ and $W 1-W 2=0.84$. The respective stellar masses of galaxies \#9 and \#59 are $5.7 \times 10^{9}$ and $5.5 \times 10^{9} M_{\odot}$, and their respective SFRs are 6.9 and $14.7 M_{\odot} \mathrm{yr}^{-1}$. However, if these galaxies indeed host an AGN, then the LePhare output parameters can only be taken as indicative since they are likely affected by larger uncertainties (the SED fits did not take into account any AGN contribution).

\subsubsection{SED fitting results for other CLASH clusters}

In our cluster sample, there are 11 CLASH clusters listed in Table 4, in which we find a total of 31 jellyfish galaxy candidates. These galaxies have optical and infrared magnitudes available (up to 17 bands between $225 \mathrm{~nm}$ and $1.6 \mu \mathrm{m}$, from the CLASH programme; see Sect. 2).

We fit SEDs of these 31 galaxies in the same way as those in MACS0717 with GAZPAR/LePhare. The best fit template spectrum includes an $\mathrm{H} \alpha$ line for all but two galaxies and, except for these two, more than half of the best fit template spectra include all main emission lines in the optical ([OII]3727, [OIII] 4959, 5007, $\mathrm{H} \beta$ and $\mathrm{H} \alpha$ ), here also suggesting that the majority of these jellyfish galaxy candidates are forming stars.

Similar plots to those shown for MACS0717 are given in the right panels of Figs. 8-12. We can see that stellar masses of these 31 galaxies cover a range comparable to that covered by the jellyfish galaxy candidates in MACS0717. Their SFRs are also comparable to those in MACS0717, but we can note that only two galaxies out of $31(6 \%)$ have SFR $>10 M_{\odot} \mathrm{yr}^{-1}$ (though one is possibly overestimated), whereas there are $25(32 \%)$ in MACS0717. Figure 12 also shows that while MACS0717 seems to be quite rich in jellyfish galaxy candidates having a high SFR, for the other clusters a clear assessment for comparison is difficult due to their incomplete coverage. The distribution of the specific star formation rate is quite different in the two samples (Fig. 10): 35 (44\%) of the galaxies in MACS0717 have SSFR $\geq 10^{-9} \mathrm{yr}^{-1}$ while the distribution of sSFRs for the 31 other jellyfish galaxy candidates is smoother, with $13(42 \%)$ galaxies having sSFR $\geq 10^{-10} \mathrm{yr}^{-1}$, though very few are quiescent (sSFR $<10^{-11} \mathrm{yr}^{-1}$ ). Consistently, the distributions of the mean ages of the stellar populations of the two samples are also quite different, as seen in Fig. 11: Half of the galaxies in 
Table 4. CLASH clusters in which the spectral energy distribution SED of candidate jellyfish galaxies was analysed.

\begin{tabular}{lc}
\hline \hline Cluster & $\begin{array}{c}\text { \# Jellyfish } \\
\text { candidates }\end{array}$ \\
\hline Abell 209 & 2 \\
Abell 383 & 3 \\
MACS J0416.1-2403 & 8 \\
MACS J0429.6-0253 & 2 \\
MACS J1149.5+2223 & 4 \\
MACS J1206.2-0847 & 3 \\
LCDCS 0829 & 1 \\
RX J1532.9+3021 & 1 \\
MACS J1931.8-2634 & 2 \\
RXC J2248.7-4431 & 5 \\
\hline
\end{tabular}

Notes. Columns are: cluster name and number of jellyfish candidates for which the SED was analysed.

MACS0717 are on average younger than $1.5 \times 10^{9} \mathrm{yrs}$, while the age distribution in the sample of 31 galaxies is flatter.

Again, we underline that these comparisons are merely indicative since the sample of 31 jellyfish galaxy candidates results from a spectroscopic and spatial coverage that is quite incomplete, and therefore by no means identical to that of MACS0717. We also looked for variations of the stellar mass, SFR, and sSFR with redshift for these 31 galaxies, but the dispersion is large, specially for the first two quantities, so we cannot claim there are clear correlations. Finally, we checked if there was a correlation between the jellyfish classification and the stellar mass, both in the 79 galaxies of MACS0717 and in the 31 galaxies belonging to other clusters, and indeed found none, in agreement with the results of Poggianti et al. (2016).

\subsection{Clusters with no jellyfish galaxy candidate identified}

There are 17 clusters in our sample in which no jellyfish galaxy candidate was detected (see Table 1). For 13 of them, there are only between one and three galaxies with a spectroscopic redshift available at the cluster redshift, and located in the zone covered by HST images. For the remaining four clusters, BMW-HRI_J122657.3+333253, ZwCl 1332.8+5043, MACS J1423+24, and Abell 2261, there are respectively 23, 6,7 , and 14 galaxies with a spectroscopic redshift available at the cluster redshift, and located in the zone covered by HST. So obviously, at least in the last three of these four clusters, the absence of jellyfish galaxies in these clusters can simply be due to the small number of available redshifts.

\section{Discussion and conclusions}

We searched for jellyfish galaxy candidates in an initial sample of 40 clusters in the redshift range $0.2<z<0.9$ from the DAFT/FADA and CLASH surveys with HST optical images available. To this purpose, two of us examined the shapes of all galaxies with a spectroscopic redshift in the approximate cluster range. This approach led us to find one or several jellyfish galaxy candidates in 23 clusters (from the original set of 40), that were classified from $J=1$ to $J=5$, following the classification scheme proposed by Ebeling et al. (2014). In the remaining 17 clusters we found no jellyfish candidate. We analysed cluster MACS0717 separately, because it has a large HST coverage and spectroscopic catalogue: In this system, we found 81 jellyfish candidates. In the remaining 22 clusters, we detect a total of 97 jellyfish galaxy candidates.

For all these jellyfish candidates, we give positions, redshifts, magnitudes in one or two optical filters (usually $F 606 \mathrm{~W}$ and $F 814 W$ ), and show images in the Appendix. Whenever images are available for the galaxies in these two wavebands, we provide both: The comparison of galaxy images in the $F 606 \mathrm{~W}$ and $F 814 W$ filters shows that our candidates are morphologically quite similar in both, but with more evidence for SF in the bluer filter, as expected.

A colour-magnitude diagram for MACS0717 shows that most of the 81 jellyfish candidates are blue and located below the cluster red sequence. This is reinforced by the UVJ diagram (Williams et al. 2009), where only two galaxies appear to be quenched; all remaining ones seem to be SF galaxies, even if several of them lie in the region of dust obscured objects (thus can be red sequence objects). For the 79 jellyfish candidates in this cluster having multi-wavelength data available, the SED fitting that was carried out with LePhare finds that almost all are best fit by template spectra that have one or several of the main optical emission lines usually associated with ongoing SF. As a consequence, the stellar mass - SFR plane (where these quantities were also obtained by LePhare directly from the best fit template spectra), shows that at least $80 \%$ of the jellyfish candidates are star-forming galaxies - and among these SF systems, about $70 \%$ have increased SFR relatively to the main sequence galaxies. The SED fit results thus provide another indication that the majority of jellyfish galaxies in this cluster have notably high SFR for their stellar masses (about $60 \%$ have sSFR $>10^{-10} \mathrm{yr}^{-1}$, stellar masses ranging from $10^{9.15}$ to $10^{10.6} M_{\odot}$ ). Though affected by the usual uncertainties associated with any SED fitting method, this result is similar to what was obtained at low redshift by Poggianti et al. (2016).

If we now look at the location of these galaxies inside the cluster, their redshift histogram does not hint for any particular placement along the line-of-sight: Jellyfish candidates share the global redshift distribution of all galaxies within the structure (i.e. all galaxies within the adopted redshift interval for this system), so it is impossible to infer any particular kinematical behaviour. As for their spatial distribution on the plane of the sky, jellyfish candidates spread throughout the cluster and its extended filament but avoid the cluster central, densest region. Since MACS0717 filaments are well detected in projection, and the redshift histogram of the whole structure (Fig. 1) is, rather surprisingly, Gaussian-like, it does look as if the main infall, at large scale, should essentially take place along the plane of the sky, in the areas where we found our jellyfish candidates.

In the cluster core, and apparently in compliance with the model of Safarzadeh \& Loeb (2019), jellyfish galaxy candidates are almost absent, which may further be a result of the very rough core environment of this massive merging cluster. A detailed study of the inner $1 \mathrm{Mpc}$ carried out with combined optical and X-ray data by Ma et al. (2009) does point to much more complex dynamics in the cluster core (when compared with what we can infer from the global redshift histogram of Fig. 1). Interestingly enough, Ellien et al. (2019) analysed the distribution of intra-cluster light (ICL) in this system and detected a large amount of it in the cluster core but no such light in the cosmic filament. As ICL is thought to be made up of disrupted galactic material, the findings of Ellien et al. (2019) corroborate the existence of a harsh environment in the core, contrasting with softer conditions along the filament. This is expected and the distribution of jellyfish galaxy candidates found in this work may reflect that, quite probably, transient phenomena such as 
jellyfish features cannot survive for long in the tumultuous core of MACS0717.

From all these pieces of evidence, we think that most jellyfish candidates identified here could be a population of rather recent infallers that have felt the first impact and effects of penetrating into a denser environment, which altered their morphology and generally increased their SFR. A tentative MIR analysis singles out two possible AGN hosts among the jellyfish candidates located in the denser cluster regions.

On the other hand, the apparent paucity of jellyfish galaxies in the cluster core could simply be the result of a selection effect, imposed by the wavelengths that we rely upon to identify them - probing, approximately, restframe B and V optical emission. Though these wavebands have been successfully used by Poggianti et al. (2016) to select their GASP jellyfish candidates at redshifts $0.04-0.07$, we expect that at the higher redshifts of our sample, the surface brightness of jellyfish structures becomes significantly dimmer. Moreover, tails and other jellyfish characteristics can indeed lie undetected in some filters since the material making up the jellyfish structures emits at selective wavelengths, as documented by several examples mentioned in Section 1. Just to highlight a couple of illustrative examples, and regardless of their location within the host cluster, we can mention FGC1287 in Abell 1367 that has a 250 kpc-long HI tail with no optical counterpart (Scott et al. 2012), and D100 in Coma, which presents a remarkably long and narrow $(60 \times 1.5 \mathrm{kpc})$ $\mathrm{H} \alpha$ gas tail, whereas the optical image shows an apparently normal spiral galaxy (Cramer et al. 2019). Such galaxies with tails are rather extreme examples of jellyfish characteristics but they seem to be located - at least in what concerns the low-redshift universe, i.e. mostly below $z=0.05$ but reaching up to $z=0.2$ - within the inner $40 \%$ of their cluster virial radius (i.e. within $1 \mathrm{Mpc}$ ) with very few exceptions (T. Scott, priv. comm.). This is the case even for those with stellar tails - that could thus potentially be unveiled in our observations. So, as far as optically detected jellyfish galaxy candidates are concerned, there do not seem to be many in the inner regions of MACS0717, at least as far as our images' depth can probe, and none shows a conspicuous tail.

Unlike the large spectroscopic coverage we have for MACS0717, which allowed us to detect in a more complete way jellyfish candidates in this cluster, the lack of redshifts in the case of most of the other clusters that we analysed prevents us from drawing major conclusions. Our aim here was simply to detect candidates, characterize them and make, whenever possible, a comparison with what was found for MACS0717. The jellyfish candidates detected in all remaining 22 clusters cover the same stellar masse range but do not seem to avoid the centre of their host clusters, as seen in Fig. 6. This might explain the generally lower SFR values (only reaching about $10 M_{\odot} \mathrm{yr}^{-1}$ ) when compared with jellyfish candidates that are members of MACS0717.

Finally, and considering the whole sample together, we next attempt to infer proportions of jellyfish galaxy candidates in the clusters analysed here and the existence of any trend with cluster relaxation states. We already mentioned the incompleteness of our redshift catalogues for the various clusters. As jellyfish galaxies are most often quite bright, and/or show emission lines in their spectra, they may be easier to observe spectroscopically, and thus their proportion may be overestimated. Besides, since our aim is to detect jellyfish galaxies, we may have classified as such galaxies that are merely somewhat strange-looking spirals with a distorted morphology. For these reasons, and due to the incompleteness of our sample, estimating the proportion of jellyfish galaxies in clusters remains difficult. We will therefore just give a few numbers. If we consider the 21 clusters for which more than ten spectroscopic redshifts are available, we find an average proportion of jellyfish galaxy candidates of $9.5 \%$ (by using the numbers in Table 1). If we now consider MACS0717, where statistics are more robust (81 jellyfish candidates detected, with a large spectroscopic and spatial coverage), we find a percentage of $13 \%$. This seems to mean that jellyfish galaxies are not that rare after all, and it is a clear encouragement to pursue such studies with more complete spectroscopic data.

Since the main mechanisms leading to jellyfish galaxies appear to be RPS and/or harassment (see the Introduction and Poggianti et al. 2017a), the proportion of jellyfish galaxies can be expected to vary with the relaxation state of clusters to which they belong. To estimate this relaxation state, we looked at several properties. First, we looked at the histograms of all the redshifts available in a large zone around each cluster (for the DAFT/FADA clusters, these histograms were given by Guennou et al. 2014 and Durret et al. 2016); for the other clusters we retrieved all the redshifts available in NED and drew their histogram in the approximate cluster redshift range. We also looked at the matter distribution based on a weak lensing analysis by Martinet et al. (2016) or on the shape of the red sequence density map by Durret et al. (2016, 2019). For clusters with at least 10 spectroscopic redshifts available within the HST images analysed in the present paper, we looked at the proportion of jellyfish galaxy candidates relative to the relaxation state of the cluster. With the available data, we found no relation between the proportion of jellyfish galaxy candidates and the relaxation state of the cluster.

Acknowledgements. It is a pleasure to thank Tom Scott for enlightening discussions about RPS and its effect on cluster galaxies. We are grateful to the referee for her/his prompt report and interesting suggestions that helped to improve the paper. F. Durret acknowledges continuous financial support from CNES since 2002. C. Lobo acknowledges support by Fundaçao para a Ciência e a Tecnologia (FCT) through the research grants UIDB/04434/2020 and UIDP/04434/2020. C. Lobo further acknowledges support from the project "Identifying the Earliest Supermassive Black Holes with ALMA (IdEaS with ALMA)" (PTDC/FISAST/29245/2017). M. Jauzac is supported by the United Kingdom Research and Innovation (UKRI) Future Leaders Fellowship 'Using Cosmic Beasts to uncover the Nature of Dark Matter' (grant number MR/S017216/1). This work is partly based on tools and data products produced by GAZPAR operated by CeSAM-LAM and IAP and we further acknowledge the dedicated support of O. Ilbert. This research has made use of the SVO Filter Profile Service (http://svo2.cab.inta-csic.es/theory/fps/) supported from the Spanish MINECO through grant AYA2017-84089. This research has also made use of the NASA/IPAC Extragalactic Database (NED), which is funded by the National Aeronautics and Space Administration and operated by the California Institute of Technology. We further acknowledge M. Taylor for developing TOPCAT, making our life so much easier.

\section{References}

Bekki, K. 2009, MNRAS, 399, 2221

Bellhouse, C., Jaffé, Y. L., McGee, S. L., et al. 2019, MNRAS, 485, 1157 Bellhouse, C., McGee, S. L., Smith, R., et al. 2021, MNRAS, 500, 1285 Bertin, E., \& Arnouts, S. 1996, A\&AS, 117, 393

Biviano, A., Rosati, P., Balestra, I., et al. 2013, A\&A, 558, A1 Boselli, A., Epinat, B., Contini, T., et al. 2019, A\&A, 631, A114 Bruzual, G., \& Charlot, S. 2003, MNRAS, 344, 1000 Chabrier, G. 2003, PASP, 115, 763

Chu, A., Durret, F., \& Marquez, I. 2021, A\&A in press, https ://doi .org/10. 1051/0004-6361/202040245

Cortese, L., Marcillac, D., Richard, J., et al. 2007, MNRAS, 376, 157

Cramer, W. J., Kenney, J. D. P., Sun, M., et al. 2019, ApJ, 870, 63

Durret, F., Márquez, I., Acebrón, A., et al. 2016, A\&A, 588, A69

Durret, F., Tarricq, Y., Márquez, I., Ashkar, H., \& Adami, C. 2019, A\&A, 622, A78

Ebeling, H., \& Kalita, B. S. 2019, ApJ, 882, 127

Ebeling, H., Stephenson, L. N., \& Edge, A. C. 2014, ApJ, 781, L40 
Ellien, A., Durret, F., Adami, C., et al. 2019, A\&A, 628, A34 Fukugita, M., Shimasaku, K., \& Ichikawa, T. 1995, PASP, 107, 945 Fumagalli, M., Fossati, M., Hau, G. K. T., et al. 2014, MNRAS, 445, 4335 George, K., Poggianti, B. M., Bellhouse, C., et al. 2019, MNRAS, 487, 3102

Girardi, M., Demarco, R., Rosati, P., \& Borgani, S. 2005, A\&A, 442, 29 Guennou, L., Adami, C., Durret, F., et al. 2014, A\&A, 561, A112

Gullieuszik, M., Poggianti, B. M., McGee, S. L., et al. 2020, ApJ, 899, 13 Gunn, J. E., Gott, J., \& Richard, I. 1972, ApJ, 176, 1

Ilbert, O., Arnouts, S., McCracken, H. J., et al. 2006, A\&A, 457, 841

Jáchym, P., Combes, F., Cortese, L., Sun, M., \& Kenney, J. D. P. 2014, ApJ, 792, 11

Jáchym, P., Kenney, J. D. P., Sun, M., et al. 2019, ApJ, 883, 145

Jauzac, M., Jullo, E., Kneib, J.-P., et al. 2012, MNRAS, 426, 3369

Jauzac, M., Harvey, D., \& Massey, R. 2018a, MNRAS, 477, 4046

Jauzac, M., Eckert, D., Schaller, M., et al. 2018b, MNRAS, 481, 2901

Kalita, B. S., \& Ebeling, H. 2019, ApJ, 887, 158

Ma, C.-J., Ebeling, H., Donovan, D., \& Barrett, E. 2008, ApJ, 684, 160

Ma, C.-J., Ebeling, H., \& Barrett, E. 2009, ApJ, 693, L56

Martinet, N., Clowe, D., Durret, F., et al. 2016, A\&A, 590, A69

Martinet, N., Durret, F., Adami, C., \& Rudnick, G. 2017, A\&A, 604, A80

Mateos, S., Alonso-Herrero, A., Carrera, F. J., et al. 2012, MNRAS, 426, 3271

McPartland, C., Ebeling, H., Roediger, E., \& Blumenthal, K. 2016, MNRAS, 455, 2994

Moore, B., Katz, N., Lake, G., Dressler, A., \& Oemler, A. 1996, Nature, 379, 613

Moretti, A., Paladino, R., Poggianti, B. M., et al. 2020, ApJ, 889, 9

Munari, E., Biviano, A., Borgani, S., Murante, G., \& Fabjan, D. 2013, MNRAS, 430, 2638

Owen, F. N., Keel, W. C., Wang, Q. D., Ledlow, M. J., \& Morrison, G. E. 2006, AJ, 131, 1974

Peng, Y.-J., Lilly, S. J., Kovač, K., et al. 2010, ApJ, 721, 193
Poggianti, B. M., Fasano, G., Omizzolo, A., et al. 2016, AJ, 151, 78 Poggianti, B. M., Moretti, A., Gullieuszik, M., et al. 2017a, ApJ, 844, 48 Poggianti, B. M., Jaffé, Y. L., Moretti, A., et al. 2017b, Nature, 548, 304 Poggianti, B. M., Ignesti, A., Gitti, M., et al. 2019, ApJ, 887, 155 Radovich, M., Poggianti, B., Jaffé, Y. L., et al. 2019, MNRAS, 486, 486 Rawle, T. D., Altieri, B., Egami, E., et al. 2014, MNRAS, 442, 196 Roman-Oliveira, F. V., Chies-Santos, A. L., Rodríguez del Pino, B., et al. 2019, MNRAS, 484, 892

Roman-Oliveira, F., Chies-Santos, A. L., Ferrari, F., Lucatelli, G., \& Rodríguez Del Pino, B. 2021, MNRAS, 500, 40

Ruggiero, R., Machado, R. E. G., Roman-Oliveira, F. V., et al. 2019, MNRAS, 484, 906

Safarzadeh, M., \& Loeb, A. 2019, MNRAS, 486, L26

Schlafly, E. F., \& Finkbeiner, D. P. 2011, ApJ, 737, 103

Schlegel, D. J., Finkbeiner, D. P., \& Davis, M. 1998, ApJ, 500, 525

Scott, T. C., Cortese, L., Brinks, E., et al. 2012, MNRAS, 419, L19

Smith, R. J., Lucey, J. R., Hammer, D., et al. 2010, MNRAS, 408, 1417

Stern, D., Assef, R. J., Benford, D. J., et al. 2012, ApJ, 753, 30

Sun, M., Donahue, M., \& Voit, G. M. 2007, ApJ, 671, 190

Taylor, M. B. 2005, in Astronomical Data Analysis Software and Systems XIV, eds. P. Shopbell, M. Britton, \& R. Ebert, ASP Conf. Ser., 347, 29

Umetsu, K., Sereno, M., Tam, S.-I., et al. 2018, ApJ, 860, 104

Vulcani, B., Poggianti, B. M., Gullieuszik, M., et al. 2018, ApJ, 866, L25

Vulcani, B., Poggianti, B. M., Moretti, A., et al. 2019, MNRAS, 487, 2278

West, M. J. 1994, MNRAS, 268, 79

West, M. J., de Propris, R., Bremer, M. N., \& Phillipps, S. 2017, Nat. Astron., 1, 0157

Williams, R. J., Quadri, R. F., Franx, M., van Dokkum, P., \& Labbé, I. 2009, ApJ, 691, 1879

Yagi, M., Yoshida, M., Komiyama, Y., et al. 2010, AJ, 140, 1814

Yoshida, M., Yagi, M., Komiyama, Y., et al. 2008, ApJ, 688, 918

Yun, K., Pillepich, A., Zinger, E., et al. 2019, MNRAS, 483, 1042 


\section{Appendix A: Images of jellyfish galaxies in}

MACS J0717.5+3745

The images of our 81 jellyfish galaxy candidates in MACS0717 are shown below. For each galaxy, we indicate the classifications estimated by two of us in parentheses (as given in Table 3).
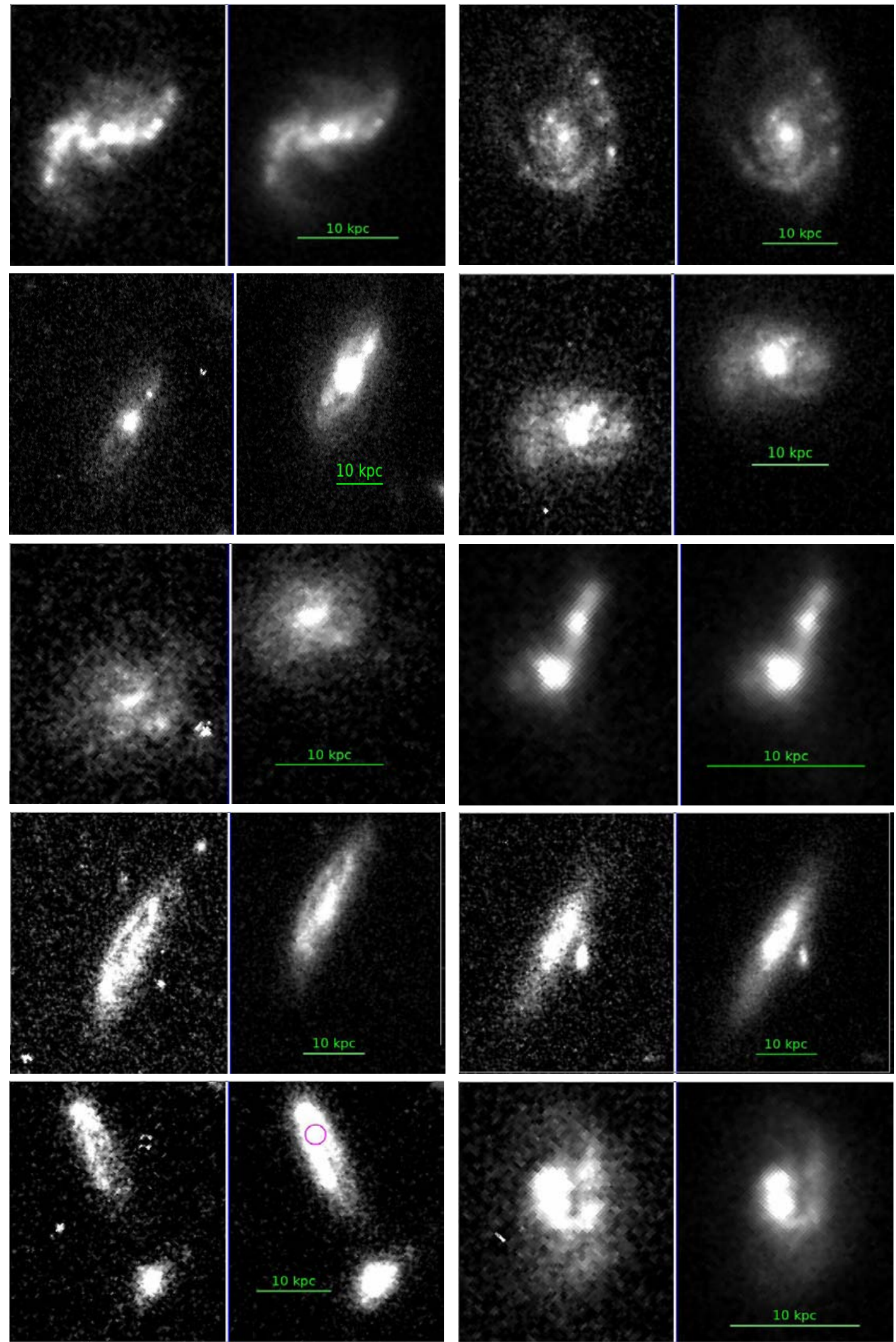

Fig. A.1. MACS J0717.5+3745. From top to bottom: galaxies \#1 (type 3-2), \#2 (3-2), \#3 (2-3), \#4 (2-1), \#5 (1-2), \#6 (1-2), \#7 (2-3), $\# 8$ (3-3), \#9 (4-4), \#10 (3-3) in the $F 606 W$ (left) and $F 814 W$ (right) filters. 

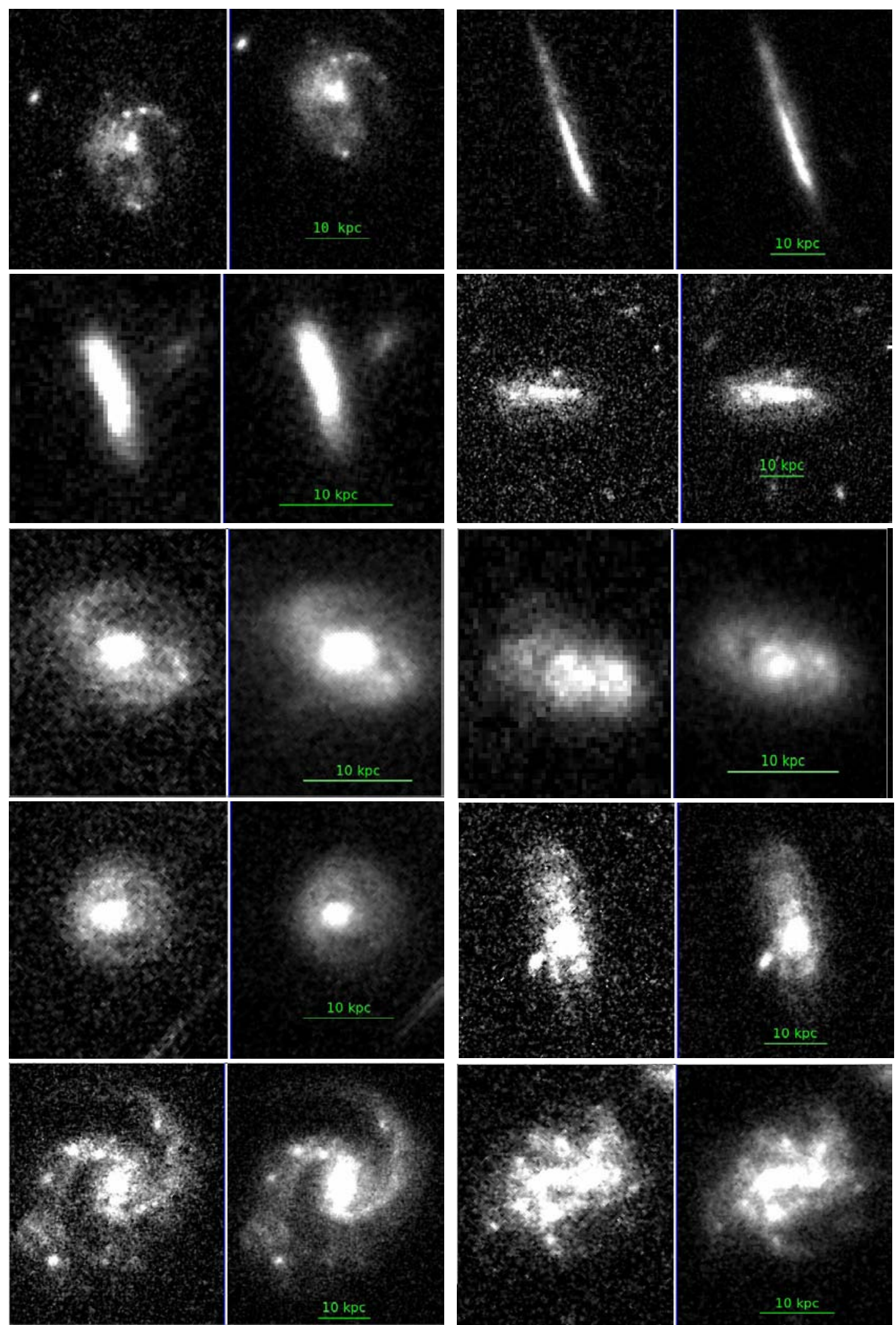

Fig. A.2. MACS J0717.5+3745. From top to bottom: galaxies \#11 (5-5), \#12 (4-4), \#13 (2-2), \#14 (2-2), \#15 (1-1), \#16 (3-2), \#17 (1-1), \#18 (4-4), $\# 19$ (2-2), \#20 (2-4) in the $F 606 W$ (left) and $F 814 W$ (right) filters. 
F. Durret et al.: Jellyfish galaxy candidates in medium redshift clusters
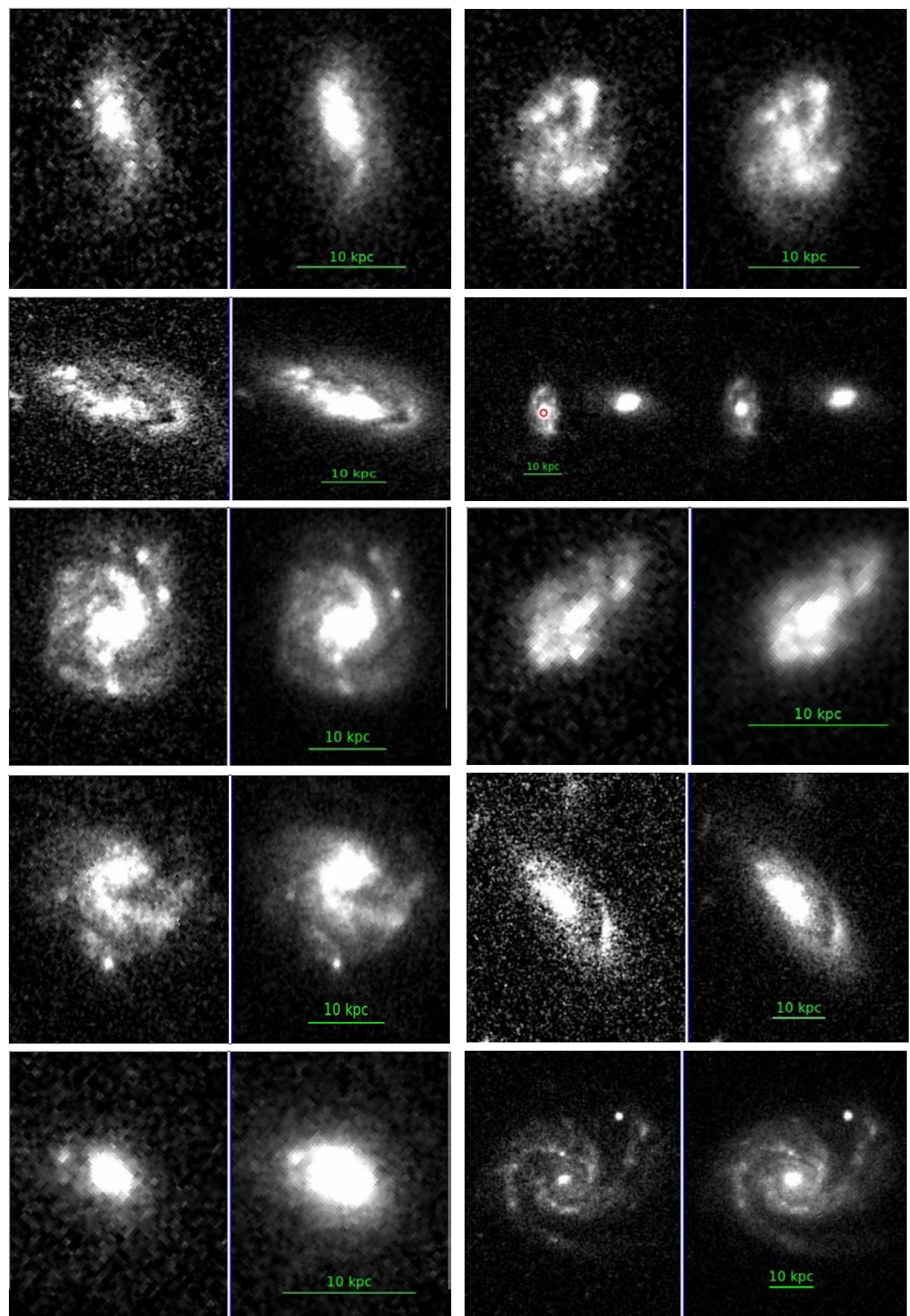

Fig. A.3. MACS J0717.5+3745. From top to bottom: galaxies \#21 (3-2), \#22 (4-4), \#23 (4-3), \#24 (3-2), \#25 (3-2), \#26 (3-3), \#27 (2-2), \#28 (1-2), \#29 (2-0), and \#30 (1-0) in the F606W (left) and F814W (right) filters. 

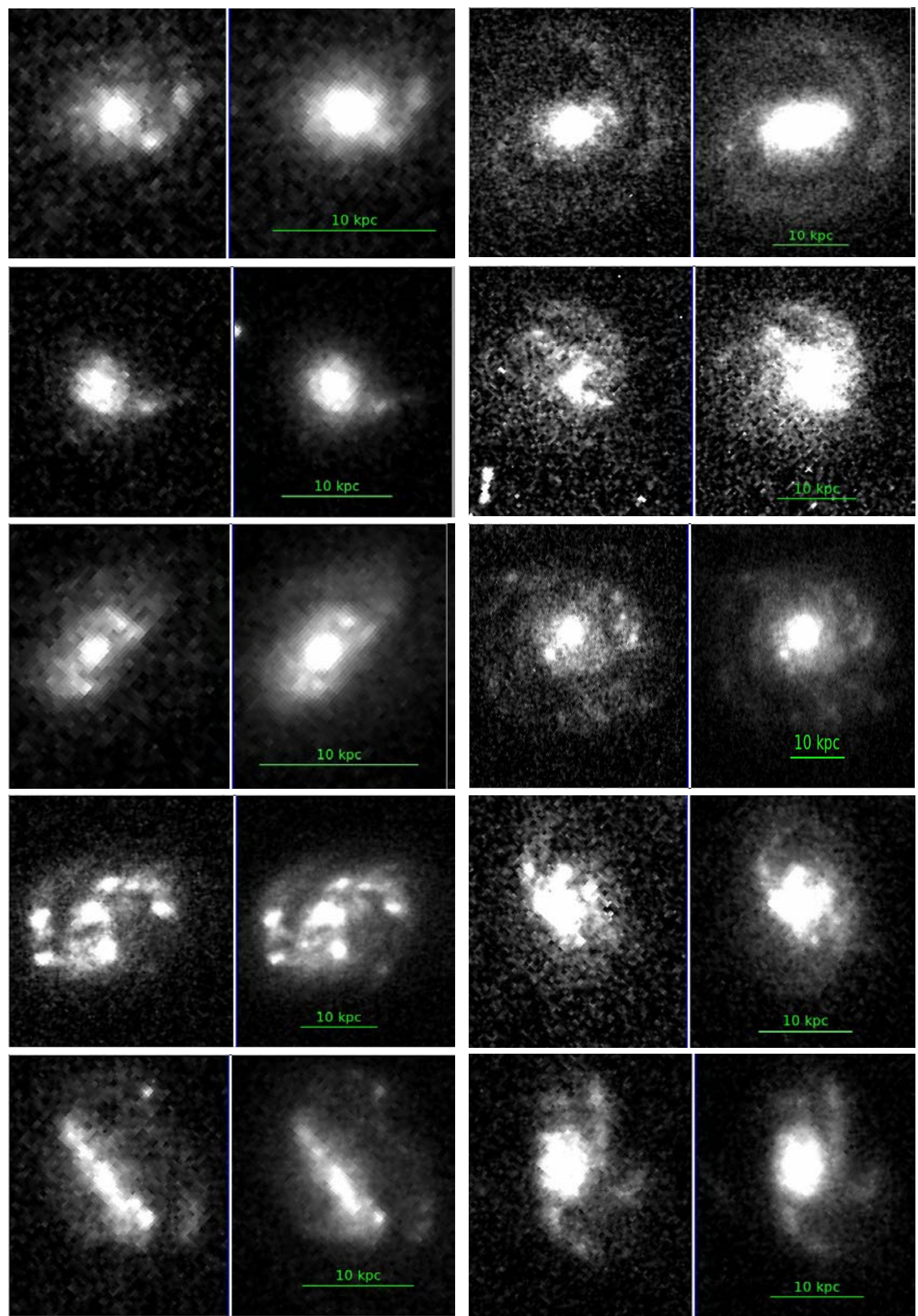

Fig. A.4. MACS J0717.5+3745. From top to bottom: galaxies \#31 (3-1), \#32 (1-0), \#33 (3-2), \#34 (2-3), \#35 (2-1), \#36 (2-2), \#37 (2-2), \#38 (4-2),\#39 (5-4), and \#40 (5-2) in the F606W (left) and $F 814 W$ (right) filters. 
F. Durret et al.: Jellyfish galaxy candidates in medium redshift clusters
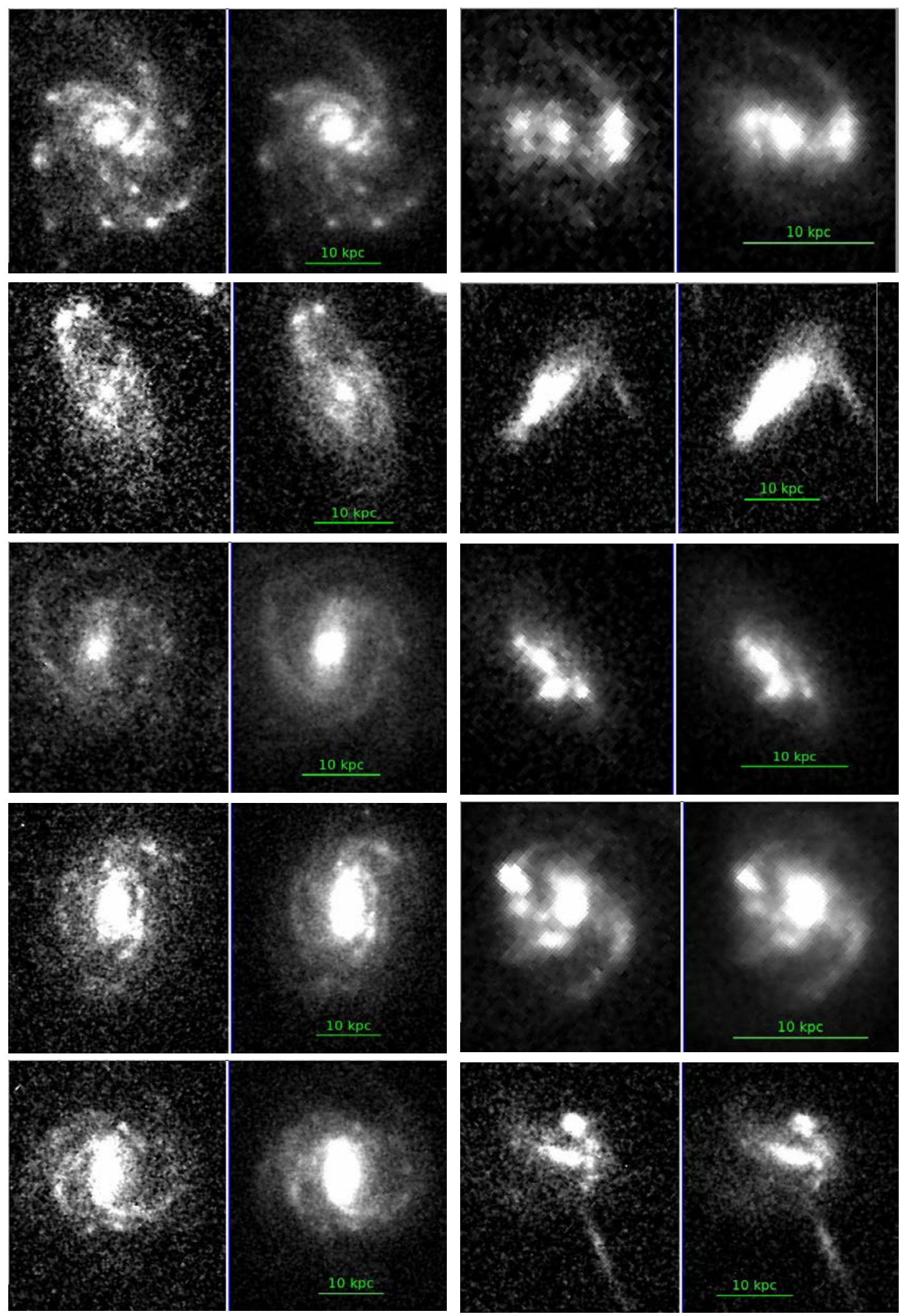

Fig. A.5. MACS J0717.5+3745. From top to bottom: galaxies \#41 (4-1), \#42 (3-3), \#43 (2-2), \#44 (5-2), \#45 (1-0), \#46 (3-2), \#47 (3-0), \#48 (4-2), \#49 (2-0), and \#50 (4-5) in the F606W (left) and F814W (right) filters. 

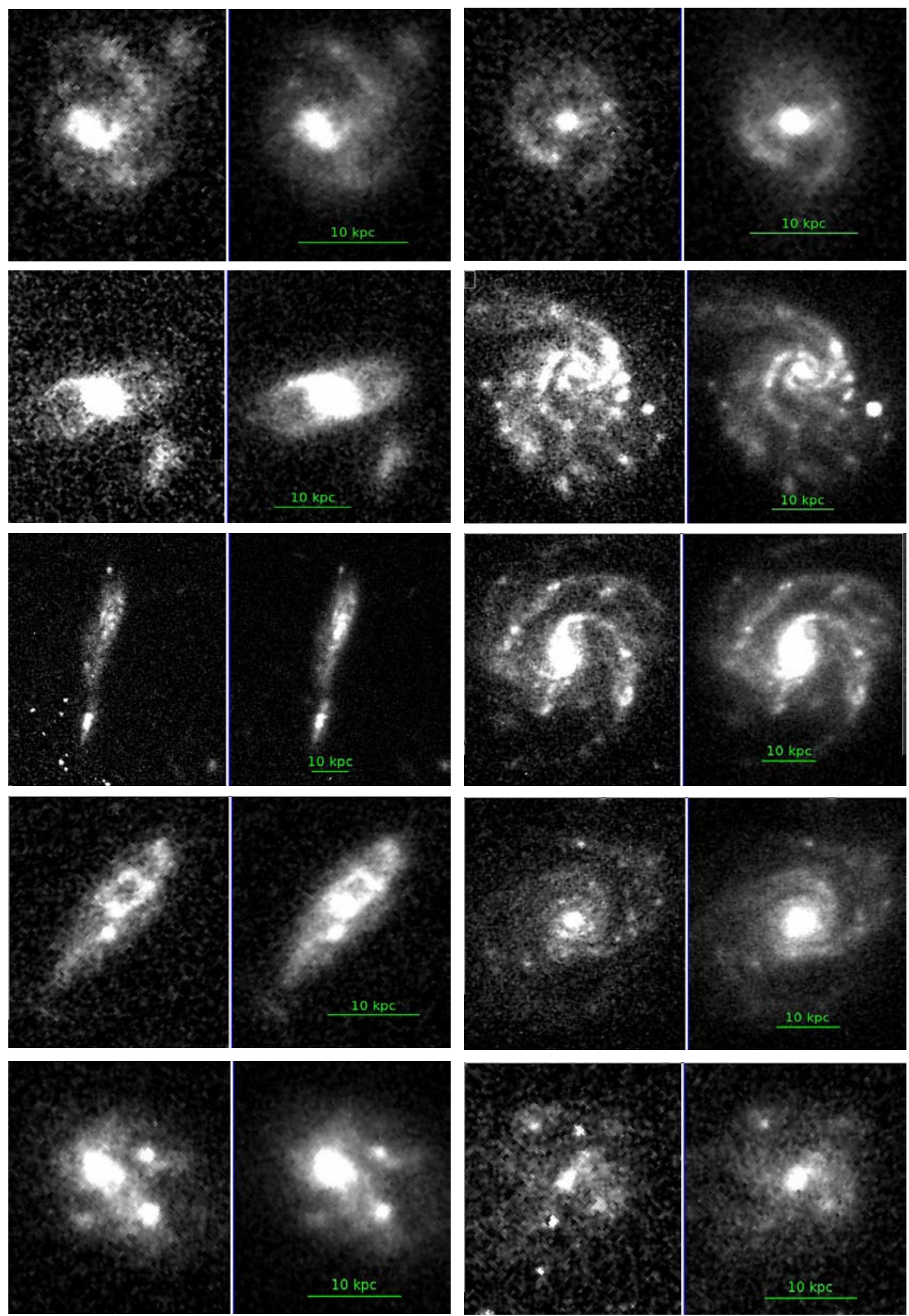

Fig. A.6. MACS J0717.5+3745. From top to bottom: galaxies \#51 (4-3), \#52 (2-0), \#53 (2-3), \#54 (5-2), \#55 (4-3), \#56 (3-1), \#57 (3-3), \#58 (1-0), $\# 59$ (3-2), and \#60 (2-2) in the $F 606 W$ (left) and F814W (right) filters. 
F. Durret et al.: Jellyfish galaxy candidates in medium redshift clusters
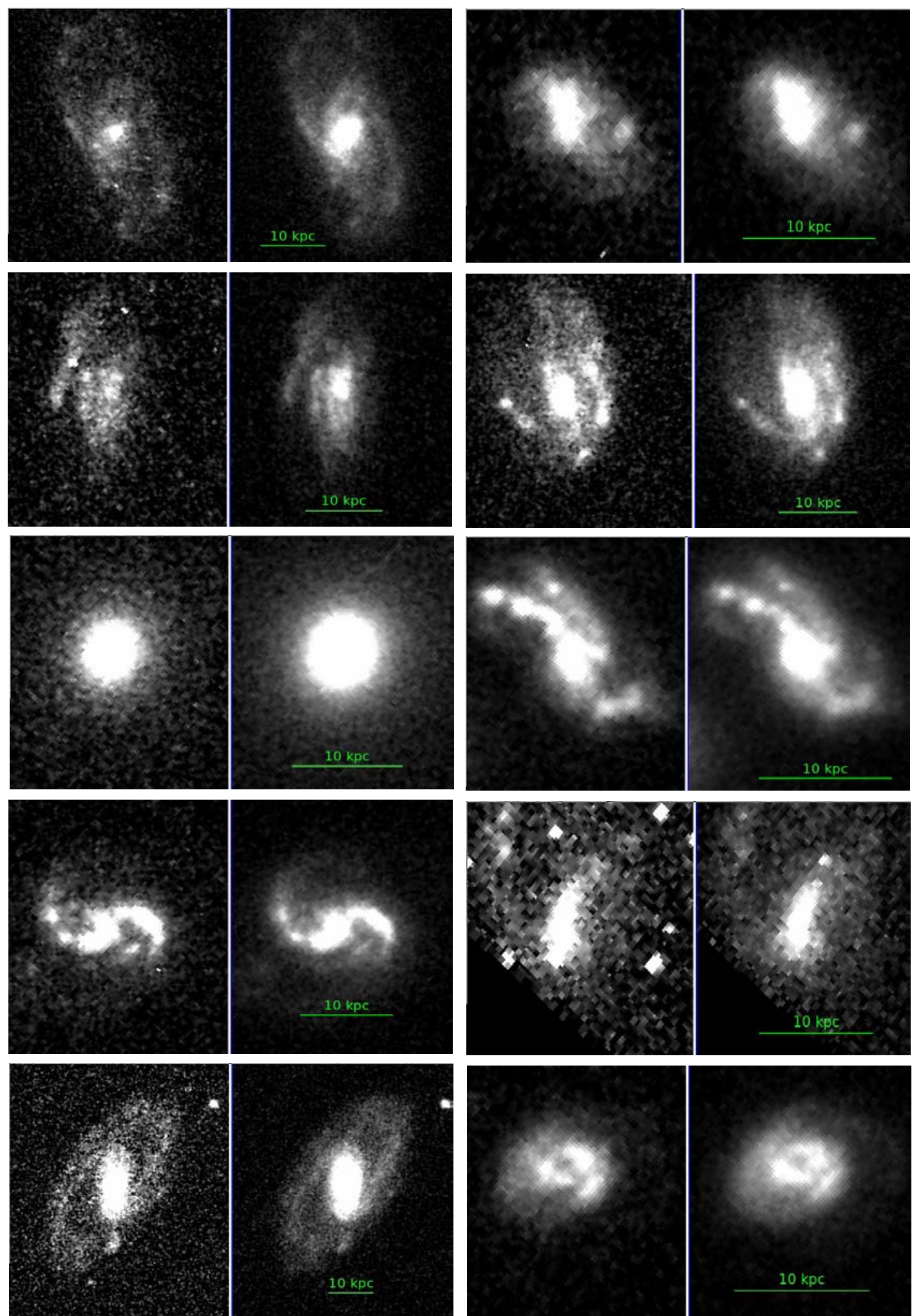

Fig. A.7. MACS J0717.5+3745. From top to bottom: galaxies \#61 (2-2), \#62 (4-2), \#63 (4-4), \#64 (5-4), \#65 (1-0), \#66 (3-2), \#67 (5-2), \#68 (4-2), $\# 69$ (2-2), and \#70 (3-2), in the F606W (left) and F814W (right) filters. 
A\&A 648, A63 (2021)
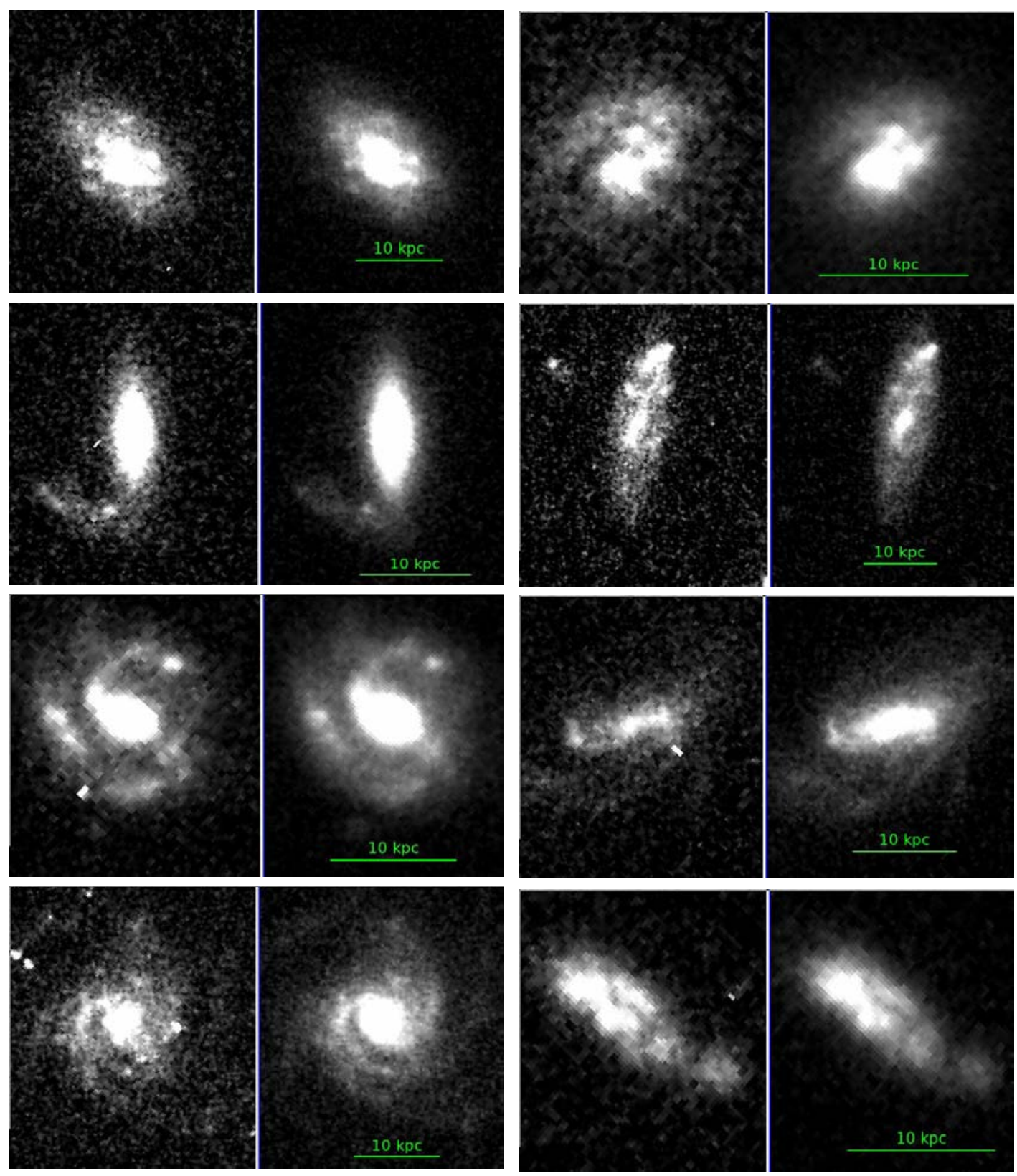

Fig. A.8. MACS J0717.5+3745. From top to bottom: galaxies \#71 (1-0), \#72 (3-2), \#73 (4-2), \#74 (2-3), \#75 (4-0), \#76 (2-2), \#77 (2-0), and \#78 (5-4) in the $F 606 W$ (left) and $F 814 W($ right) filters. 
F. Durret et al.: Jellyfish galaxy candidates in medium redshift clusters
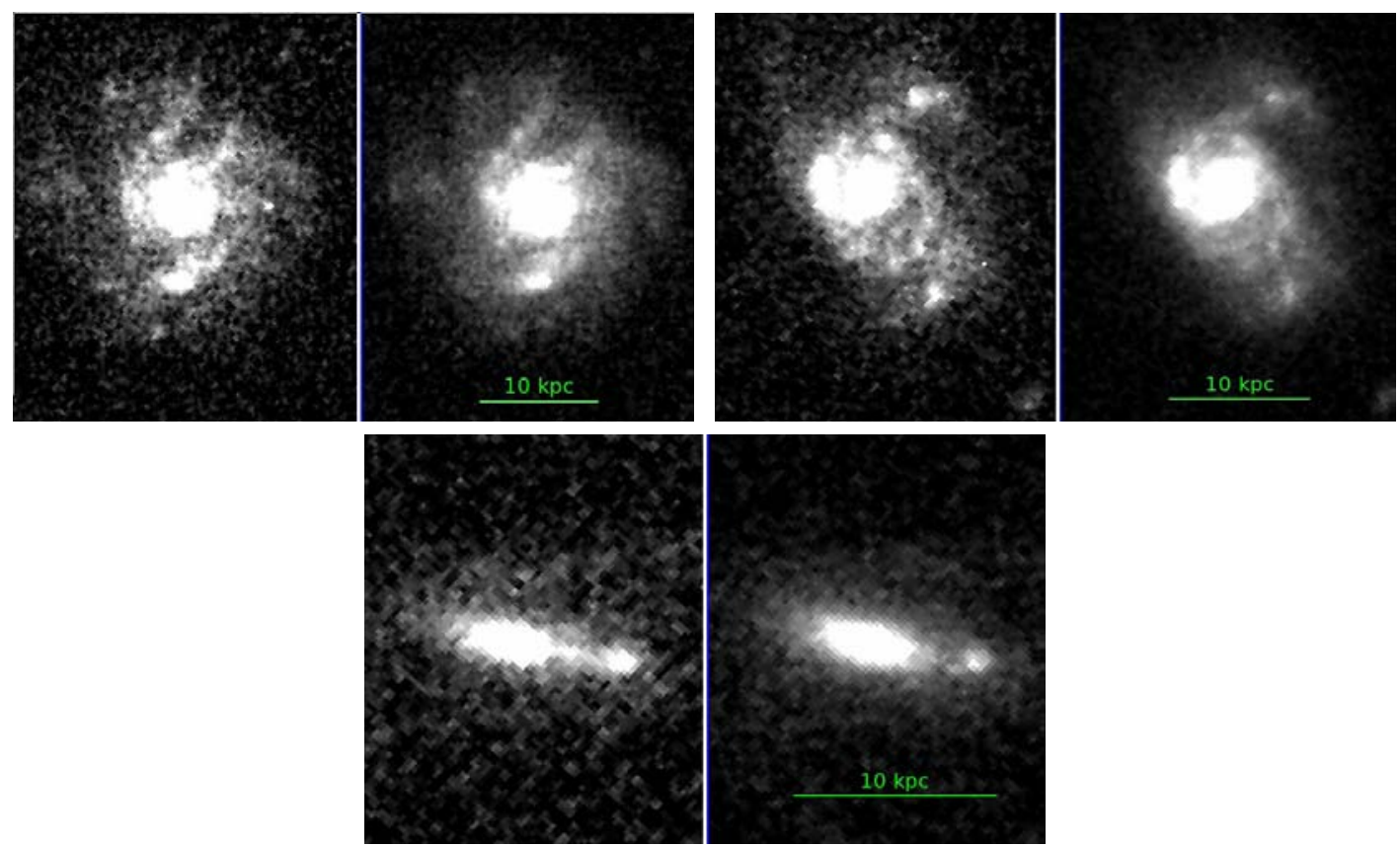

Fig. A.9. MACS J0717.5+3745. From top to bottom: galaxies \#79 (4-3), \#80 (4-4) and \#81 (3-2) in the F606W (left) and F814W (right) filters.

Appendix B: Images of jellyfish galaxies in all clusters except MACS J0717.5+3745
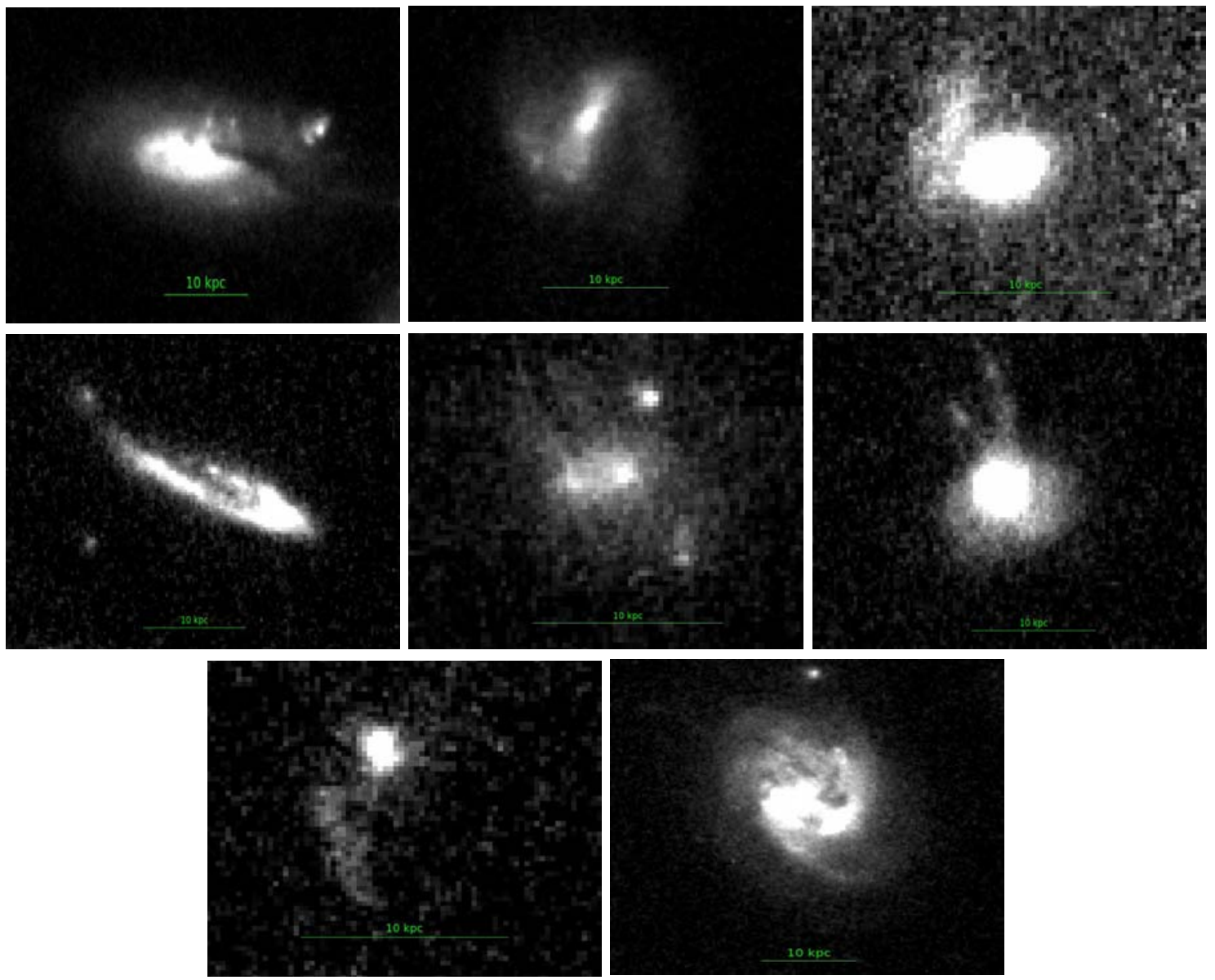

Fig. B.1. Cl0016+16 $(z=0.5455)$. All the images are in F814W. Row 1: Galaxies a, b, and c. Row 2: Galaxies d, e, and f. Row 3: Galaxies g and h. 


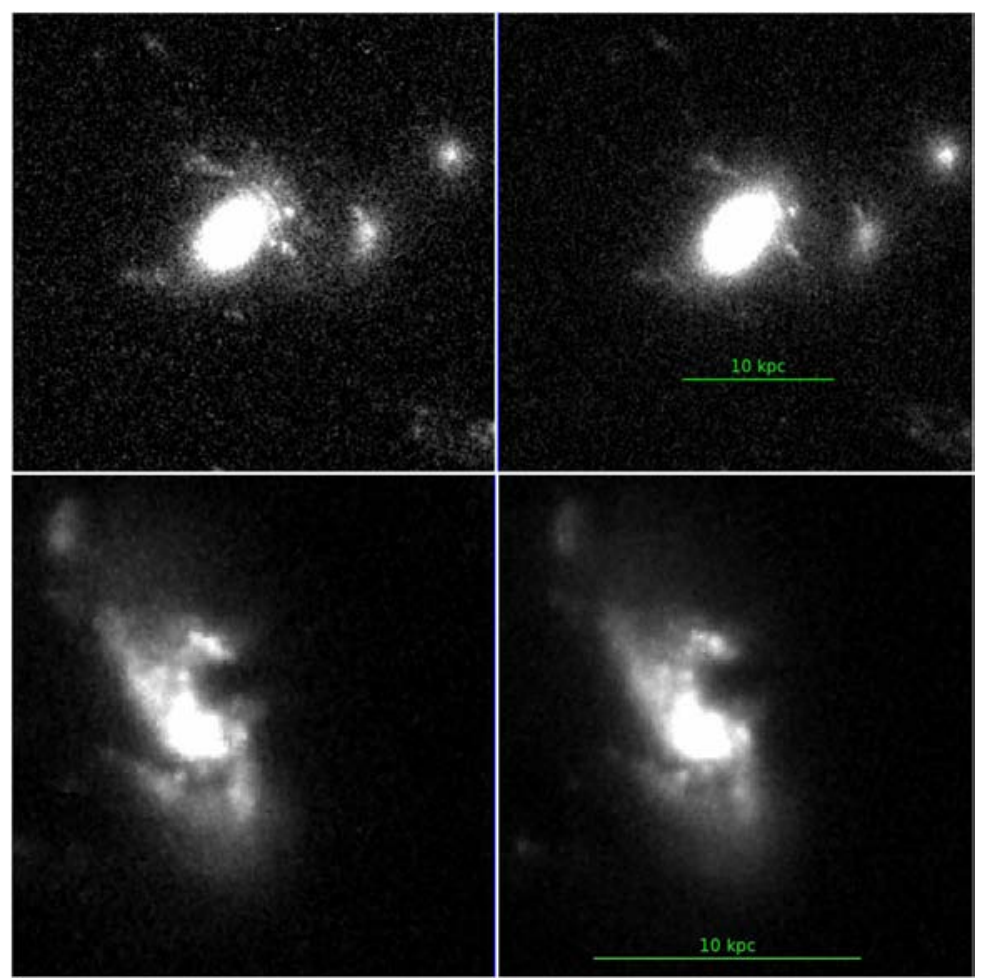

Fig. B.2. A209 $(z=0.206)$. Row 1: Galaxy a in $F 606 W$ and $F 814 W$. Row 2: Galaxy b in $F 606 W$ and $F 814 W$.
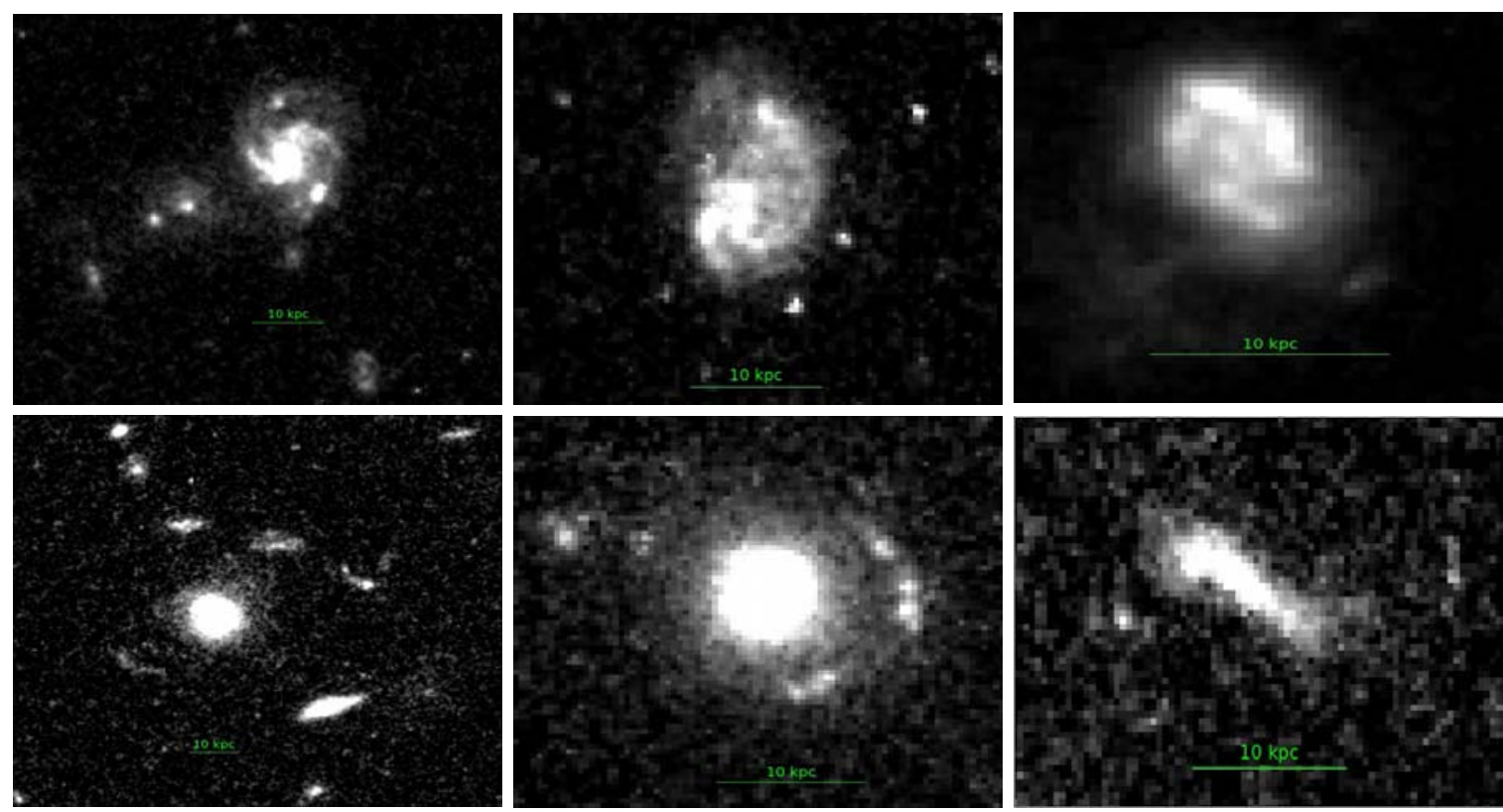

Fig. B.3. C10152.7-1357 $(z=0.831)$. All the images are in F775W. Row 1: Galaxies a, b, and c. Row 2: Galaxies d, e, and f. 
F. Durret et al.: Jellyfish galaxy candidates in medium redshift clusters

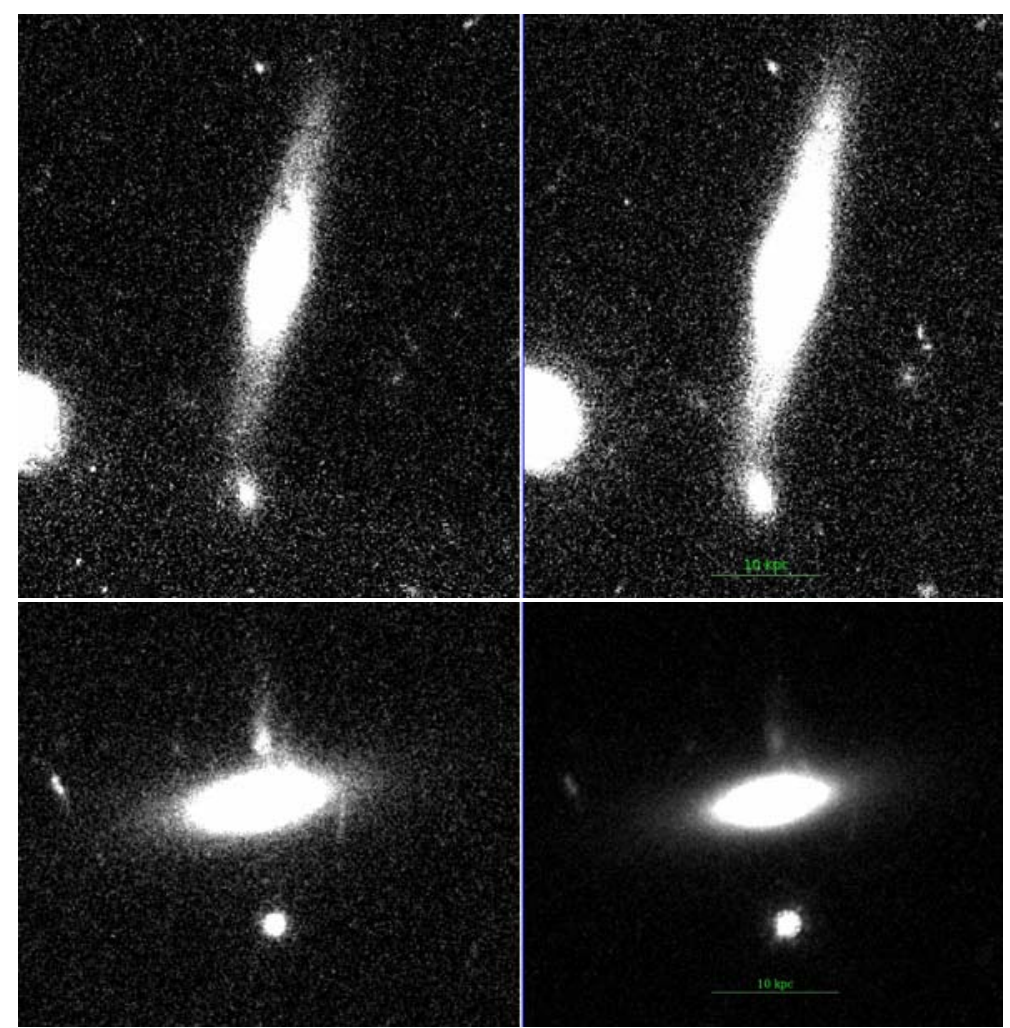

Fig. B.4. A383 $(z=0.1871)$ in $F 606 W(l e f t)$ and $F 814 W($ right $)$. Row 1: Galaxy a, row 2: Galaxy b. 
A\&A 648, A63 (2021)


Fig. B.5. MACS0416 $(z=0.396)$ in $F 606 W$ and $F 814 W$. Row 1: Galaxies a and b. Row 2: Galaxies c and d. Row 3: Galaxies e and f. Row 4: Galaxies g and $\mathrm{h}$ (green circle).
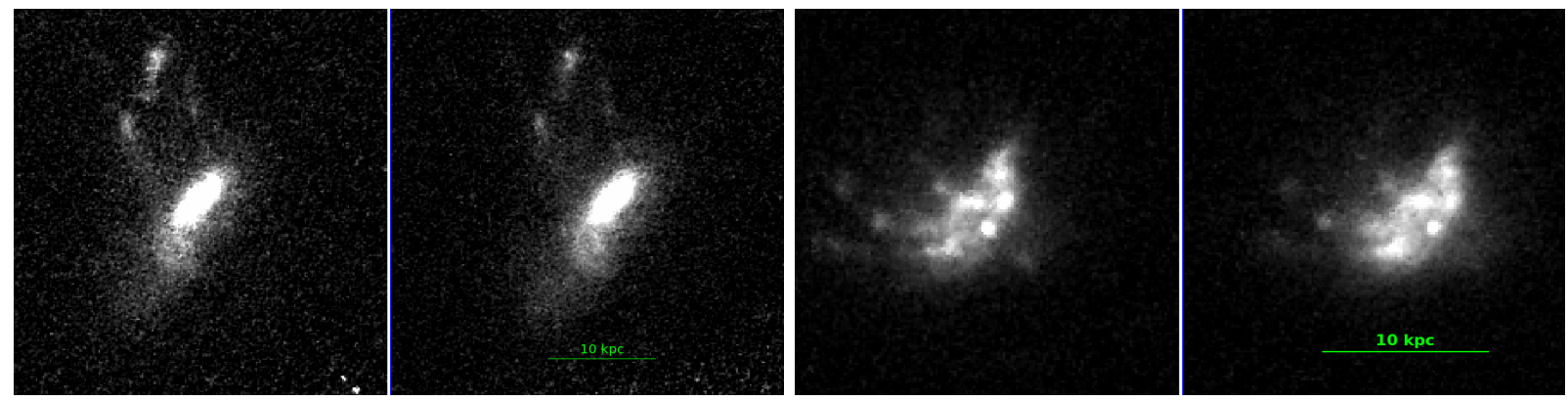

Fig. B.6. MACS0429 $(z=0.399)$ in $F 606 W$ and $F 814 W$. Left: Galaxy a, right: Galaxy b. 
F. Durret et al.: Jellyfish galaxy candidates in medium redshift clusters
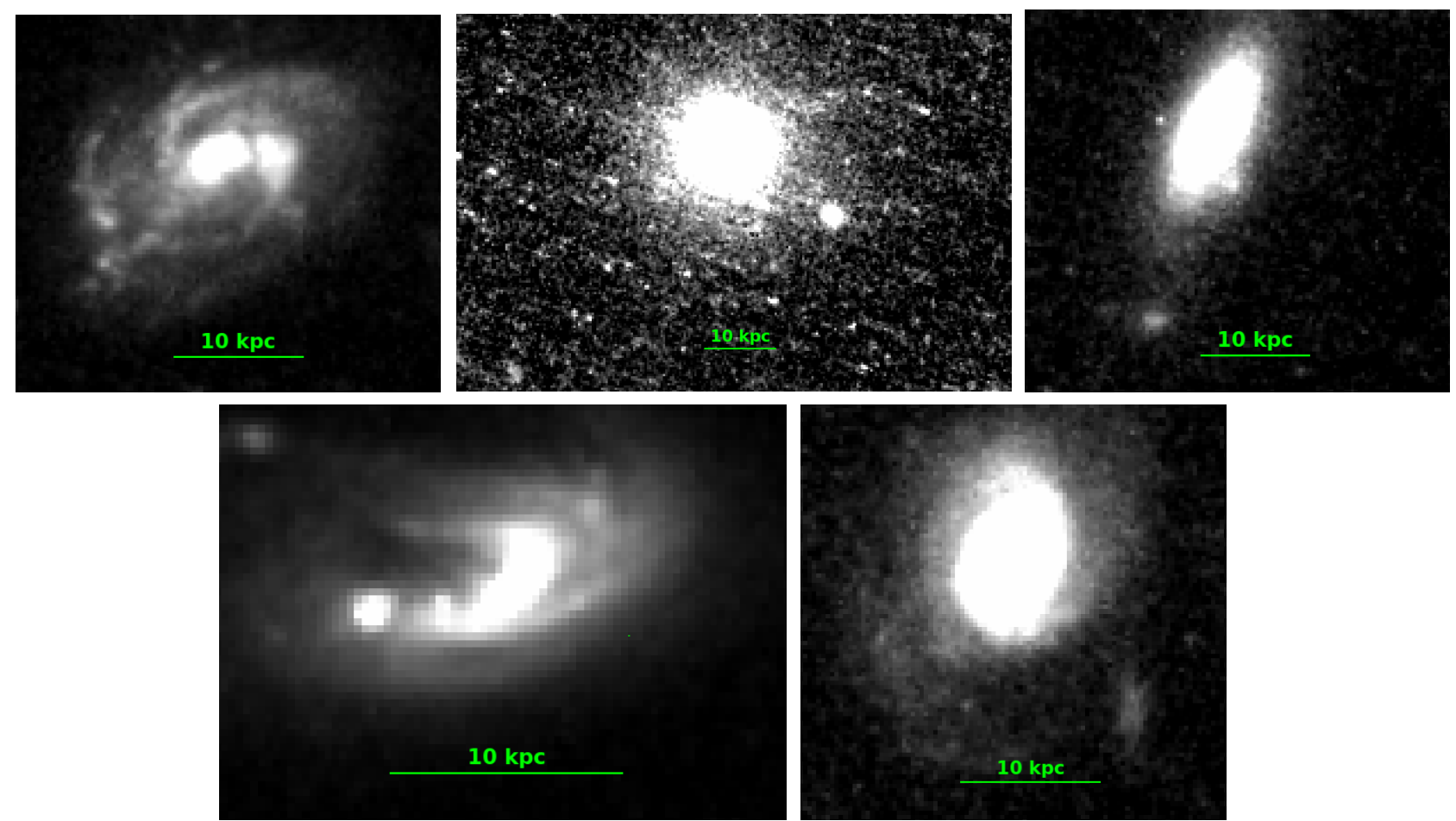

Fig. B.7. MACS0454 $(z=0.5377)$. All galaxies are in F814W. Row 1: Galaxies a, b, and c. Row 2: Galaxies d and e. 

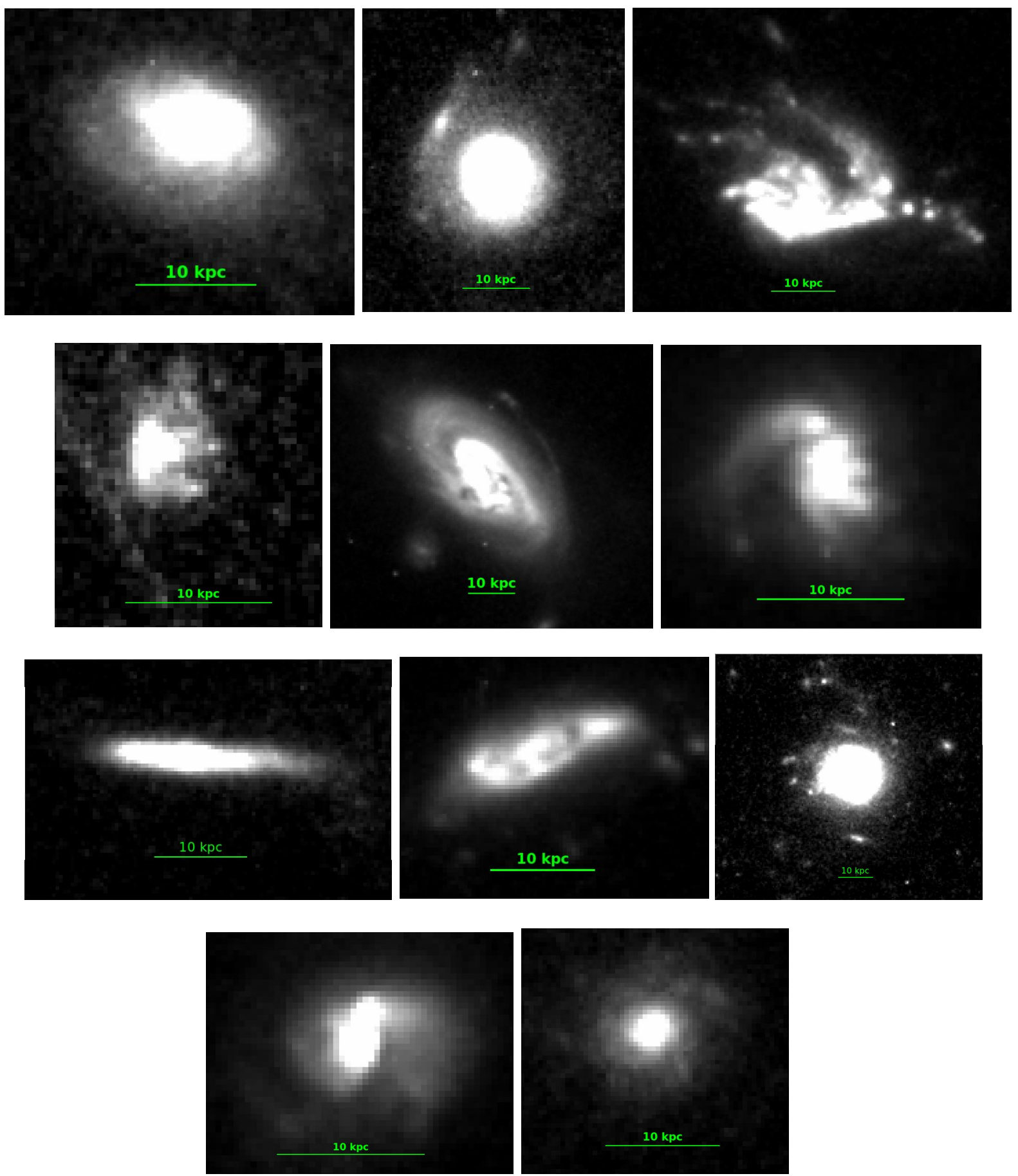

Fig. B.8. A851 $(z=0.4069)$. All the images are in F814W. Row 1: Galaxies a, b, and c. Row 2: Galaxies d, e, and f. Row 3: Galaxies g, h, and i. Row 4: Galaxies j and k. 
F. Durret et al.: Jellyfish galaxy candidates in medium redshift clusters
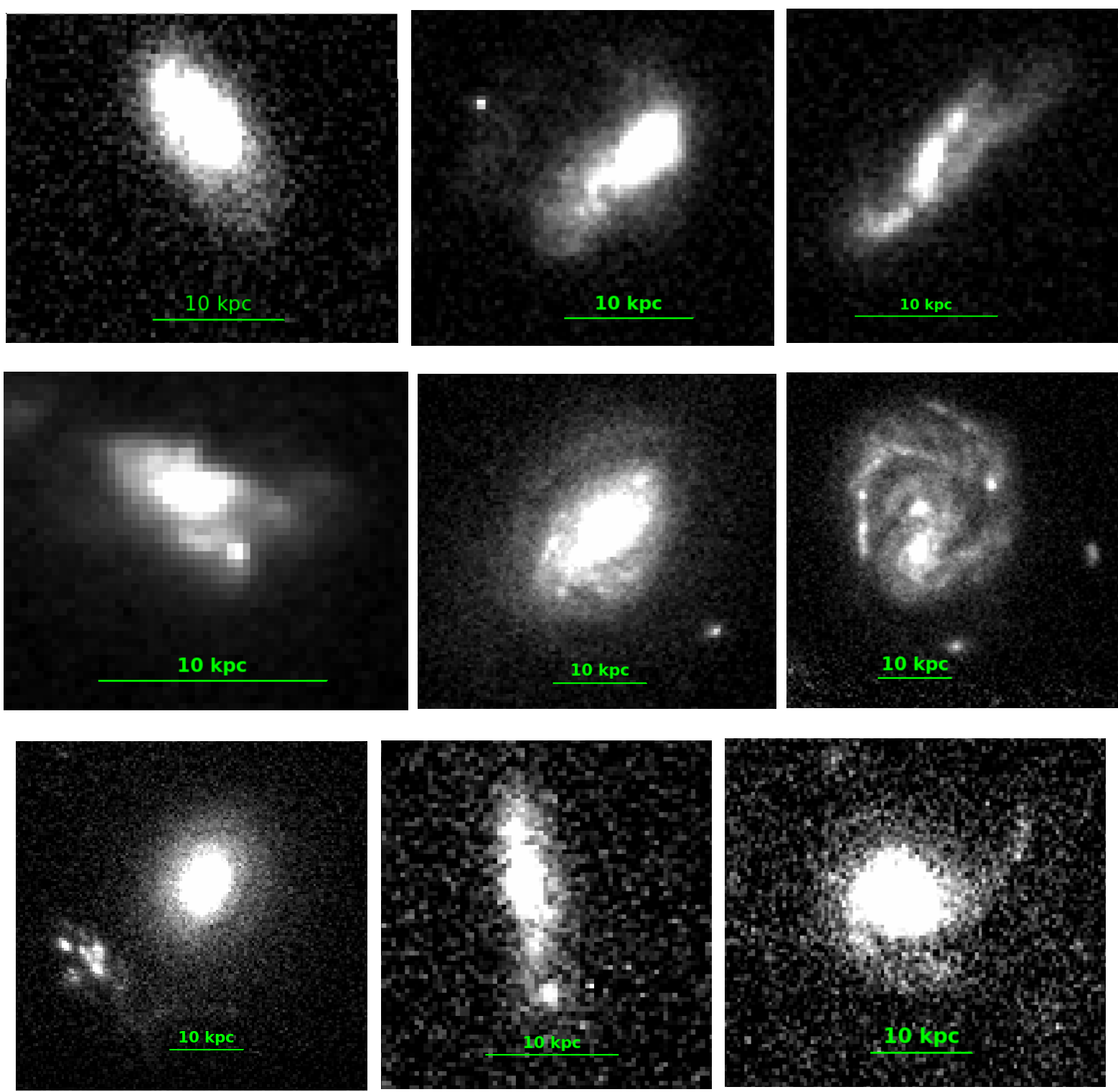

Fig. B.9. LCDCS0172 $(z=0.6972)$. All the images are in F814W. Row 1: Galaxies a, b, and c. Row 2: Galaxies d, e, and f. Row 3: Galaxies g, h, and $i$.
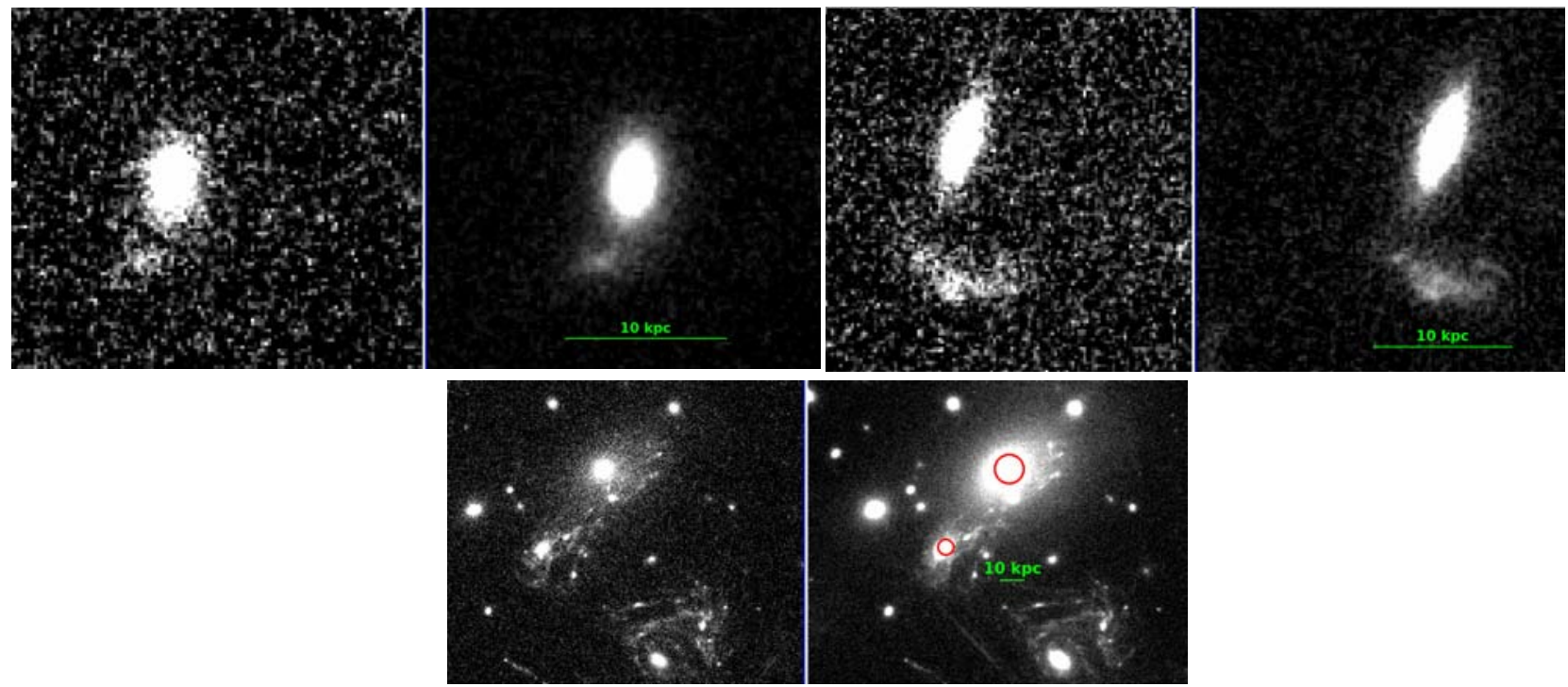

Fig. B.10. MACS1 $149(z=0.544)$. All the images are in $F 606 W$ and $F 814 W$. Row 1: Galaxies a and b. Row 2: Galaxies c and d (both are at the cluster redshift, $\mathrm{c}$ is to the right). 


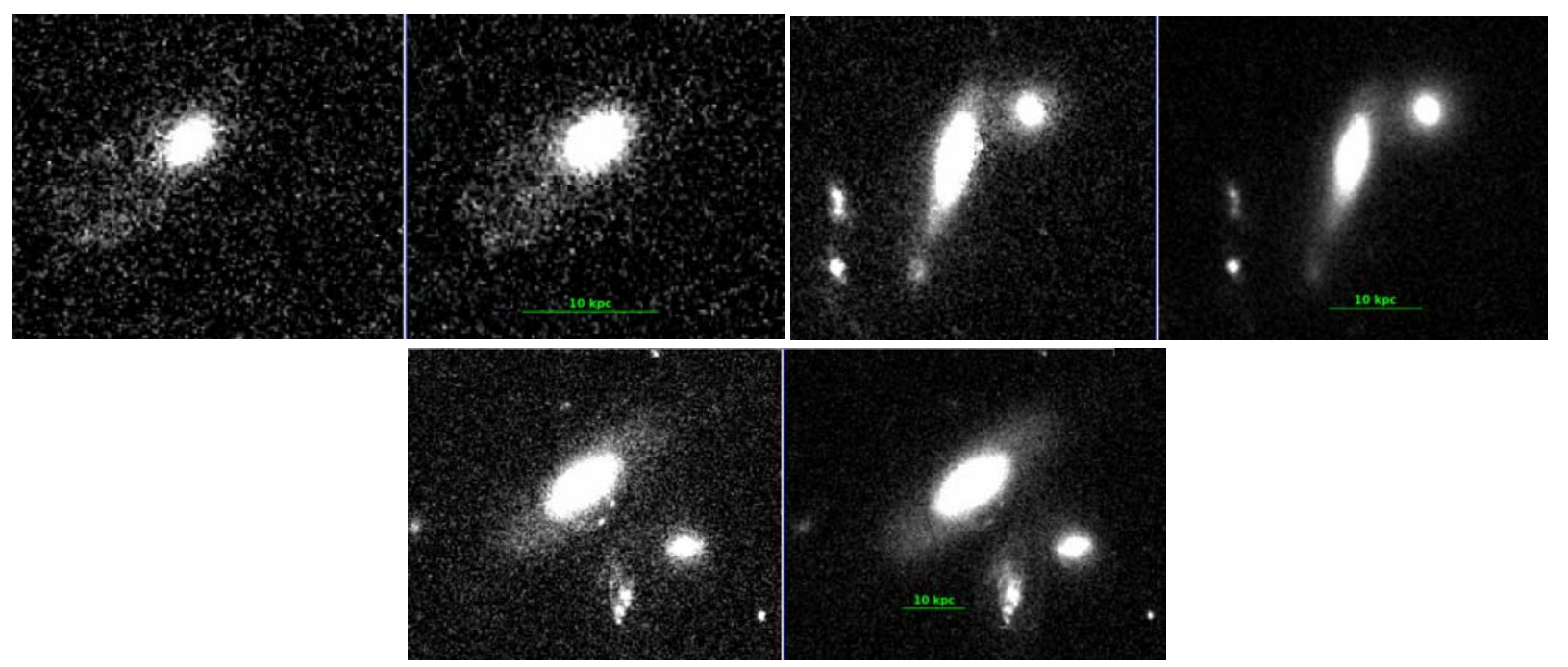

Fig. B.11. MACS1206 $(z=0.44)$. All the images are in F606W and F814W. Row 1: Galaxies a and b. Row 2: Galaxy c.
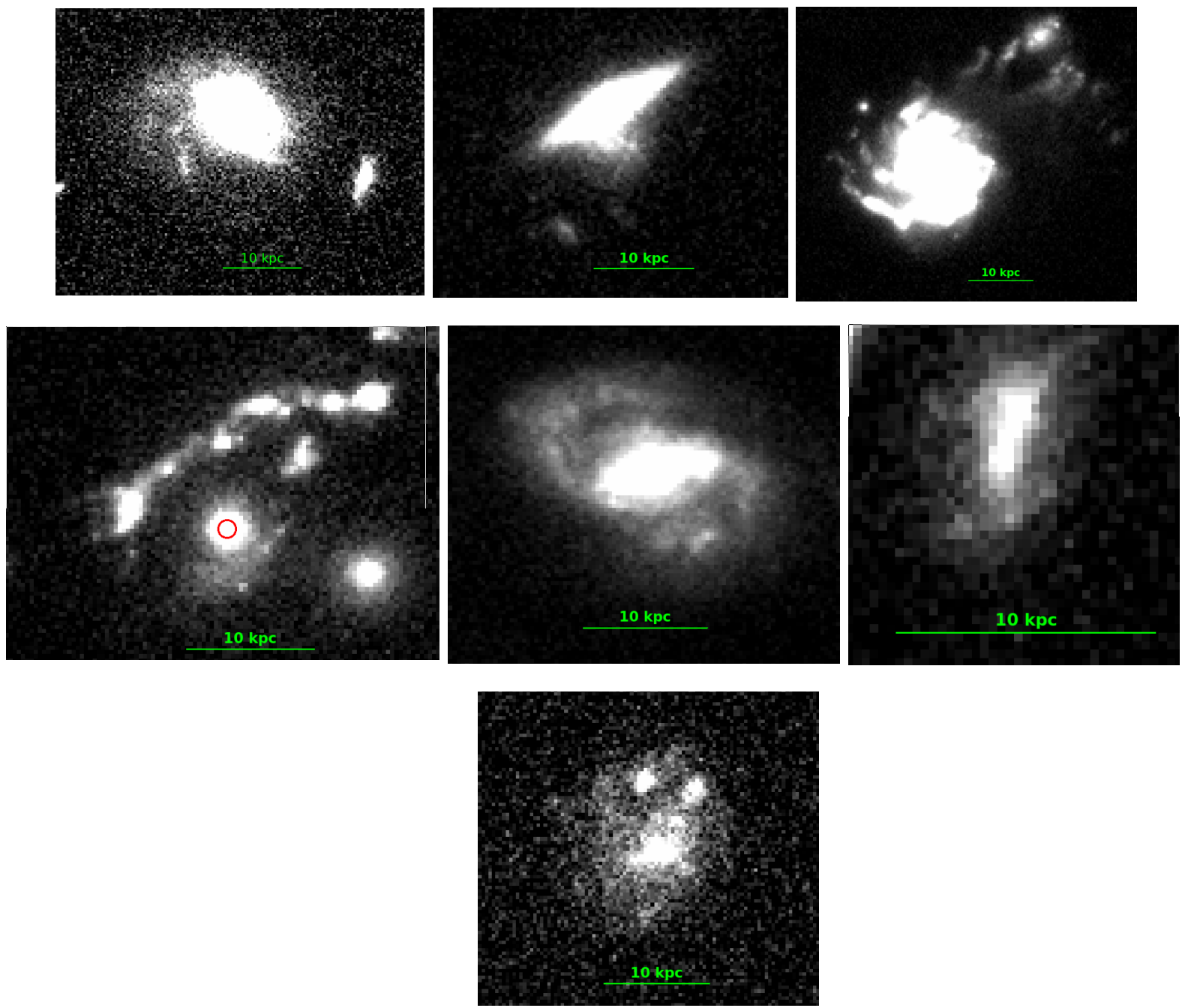

Fig. B.12. LCDCS0541 $(z=0.5414)$. All images are in F814W. Row 1: Galaxies a, b, and c. Row 2: Galaxies d (red circle), e, and f. Row 3: Galaxy g. 
F. Durret et al.: Jellyfish galaxy candidates in medium redshift clusters

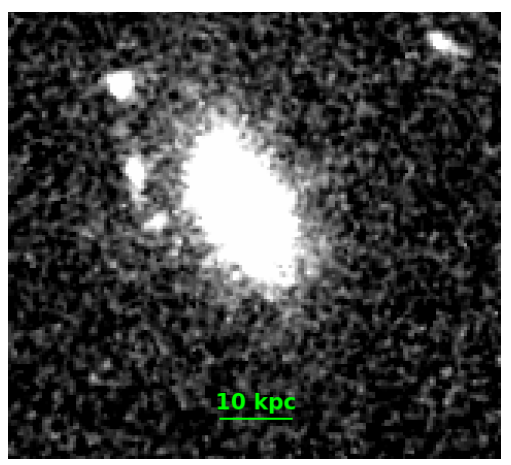

Fig. B.13. MJM98_034 $(z=0.595)$. Galaxy a is in $F 712 W$.

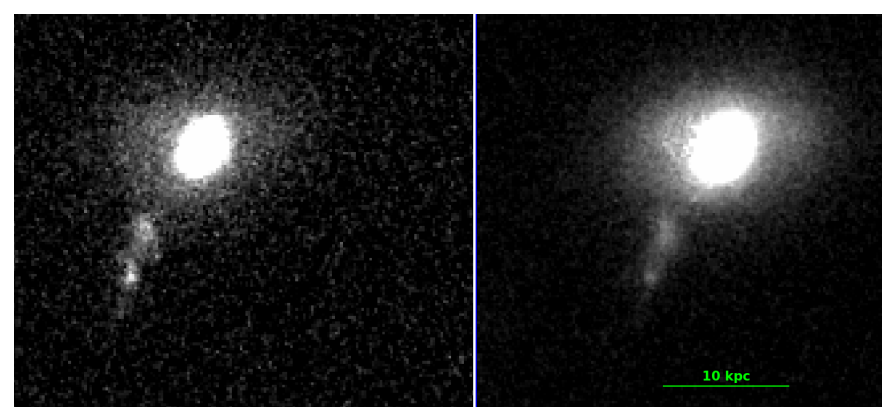

Fig. B.14. LCDCS0829 $(z=0.451)$. Galaxy a is in $F 606 W$ and $F 814 W$.
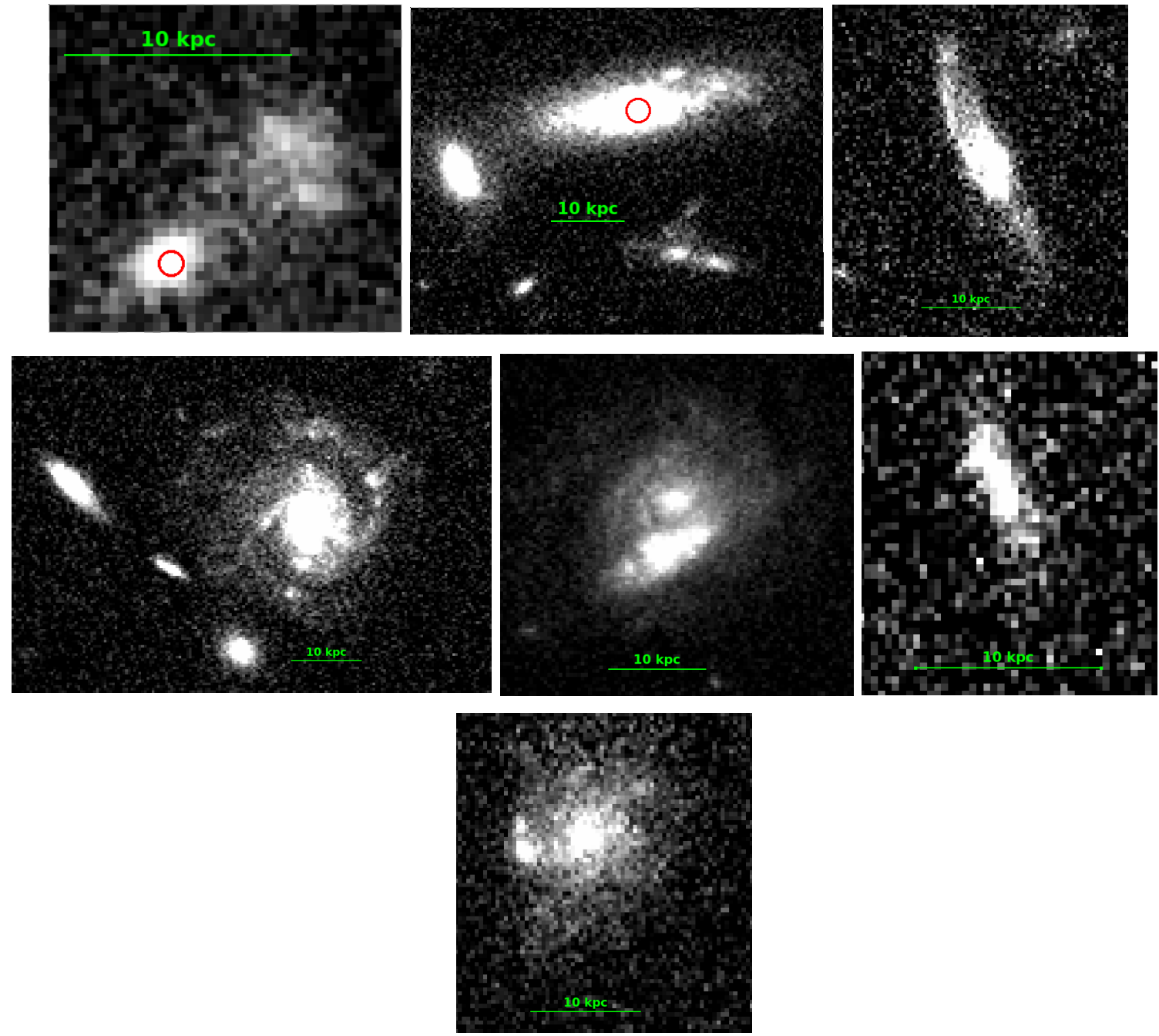

Fig. B.15. LCDCS0853 ( $z=0.7627)$. All galaxies are in $F 814 W$. Row 1: Galaxies a (red circle), b (red circle), and c. Row 2: Galaxies d, e, and f. Row 3: Galaxy g. 

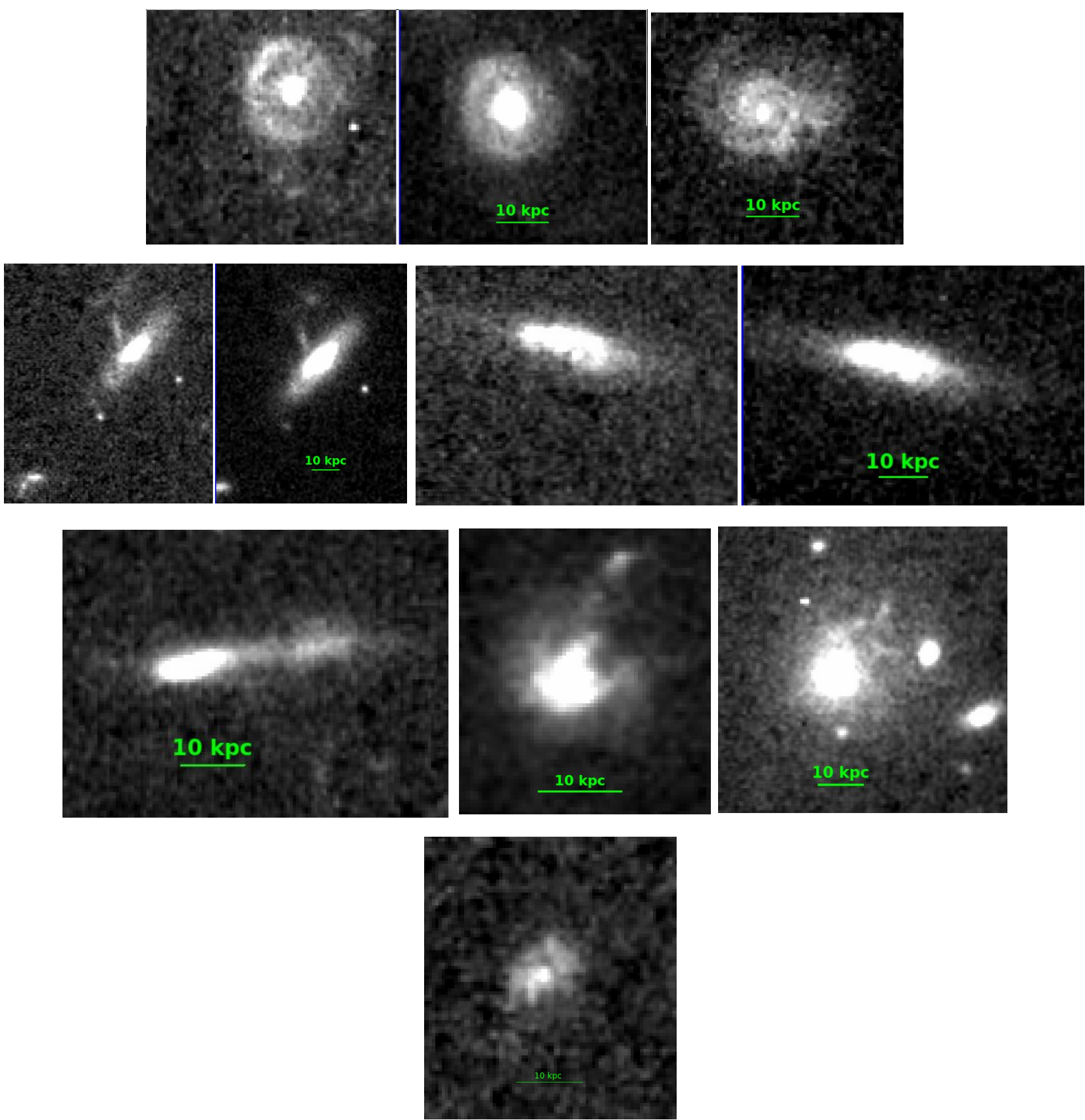

Fig. B.16. 3C295 $(z=0.46)$. Row 1: Galaxy a in $F 555 W$ and $F 814 W$, and Galaxy b in $F 814 W$. Row 2: Galaxies $\mathrm{c}$ and $\mathrm{d}$ in $F 555 W$ and $F 814 W$. Row 3: Galaxies e, f, and g in F555W. Row 4: Galaxy h in F555W.
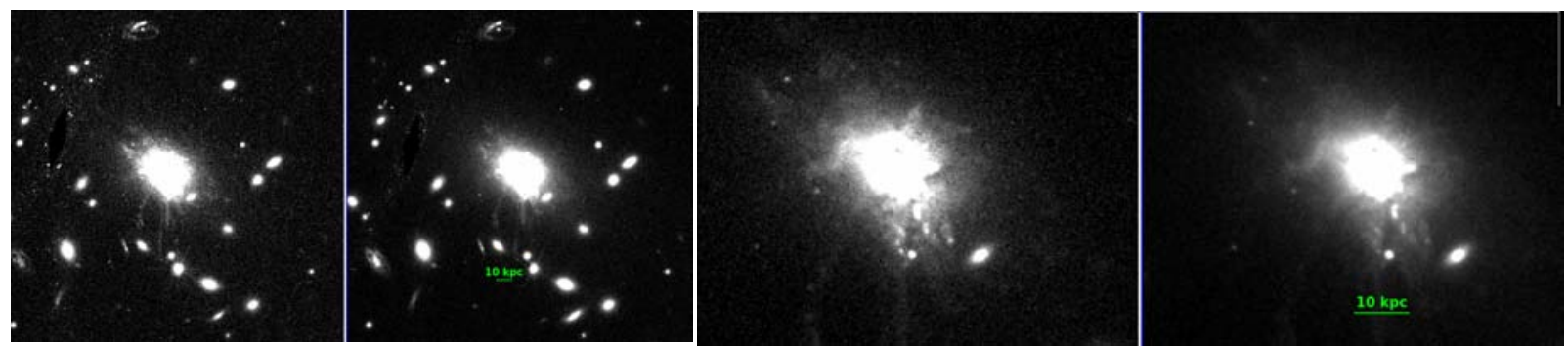

Fig. B.17. RX1532 $(z=0.345)$. Images are in $F 606 W$ and $F 814 W$. Left: galaxy a, which is the BCG, showing filaments reminiscent of the Perseus cluster BCG. Right: zoom-in on galaxy a. 

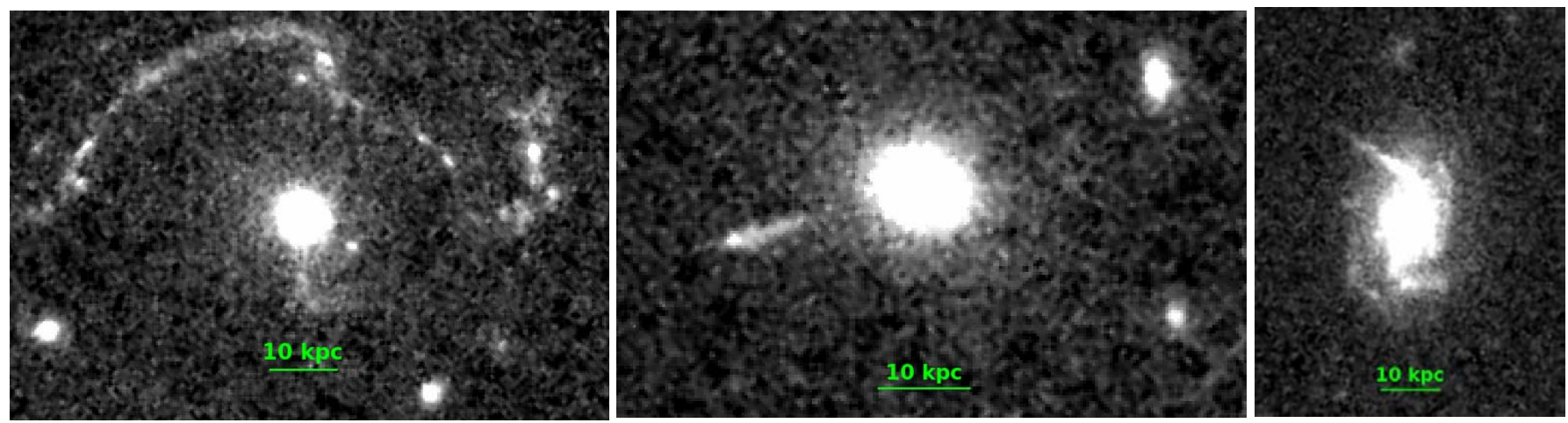

Fig. B.18. MS1621 $(z=0.426)$. Galaxies a, b, and c are in $F 814 W$.

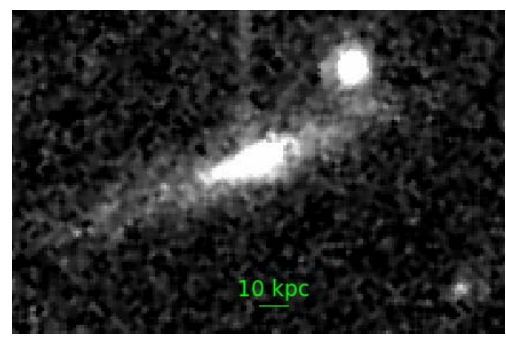

Fig. B.19. RX1716 $(z=0.813)$, galaxy a is in $F 814 W$.

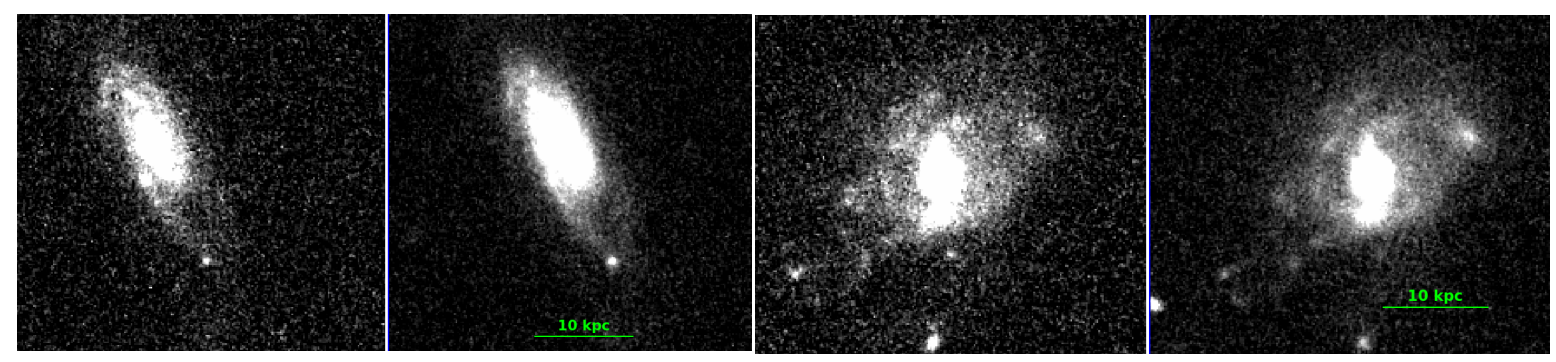

Fig. B.20. MACS1931 $(z=0.352)$. Images are in $F 606 W$ and $F 814 W$. Left: galaxy a, right: galaxy b.

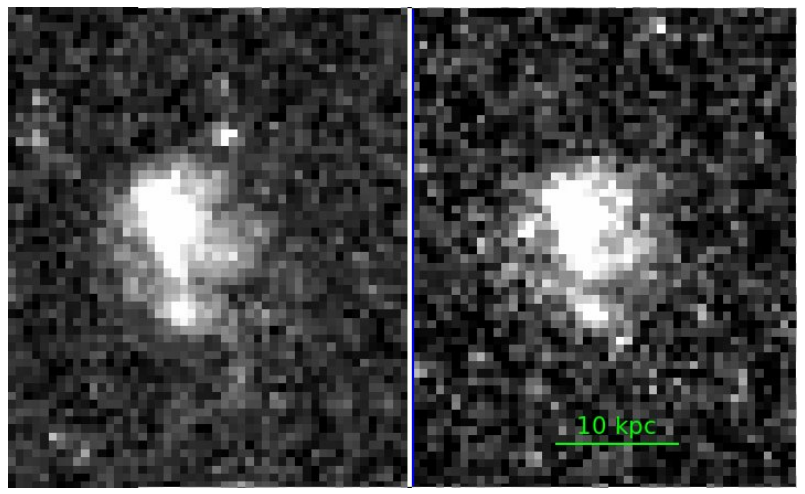

Fig. B.21. MS 2053.7-0449 $(z=0.583)$. Images of galaxy a in $F 606 W(l e f t)$ and $F 814 W($ right $)$. 
A\&A 648, A63 (2021)
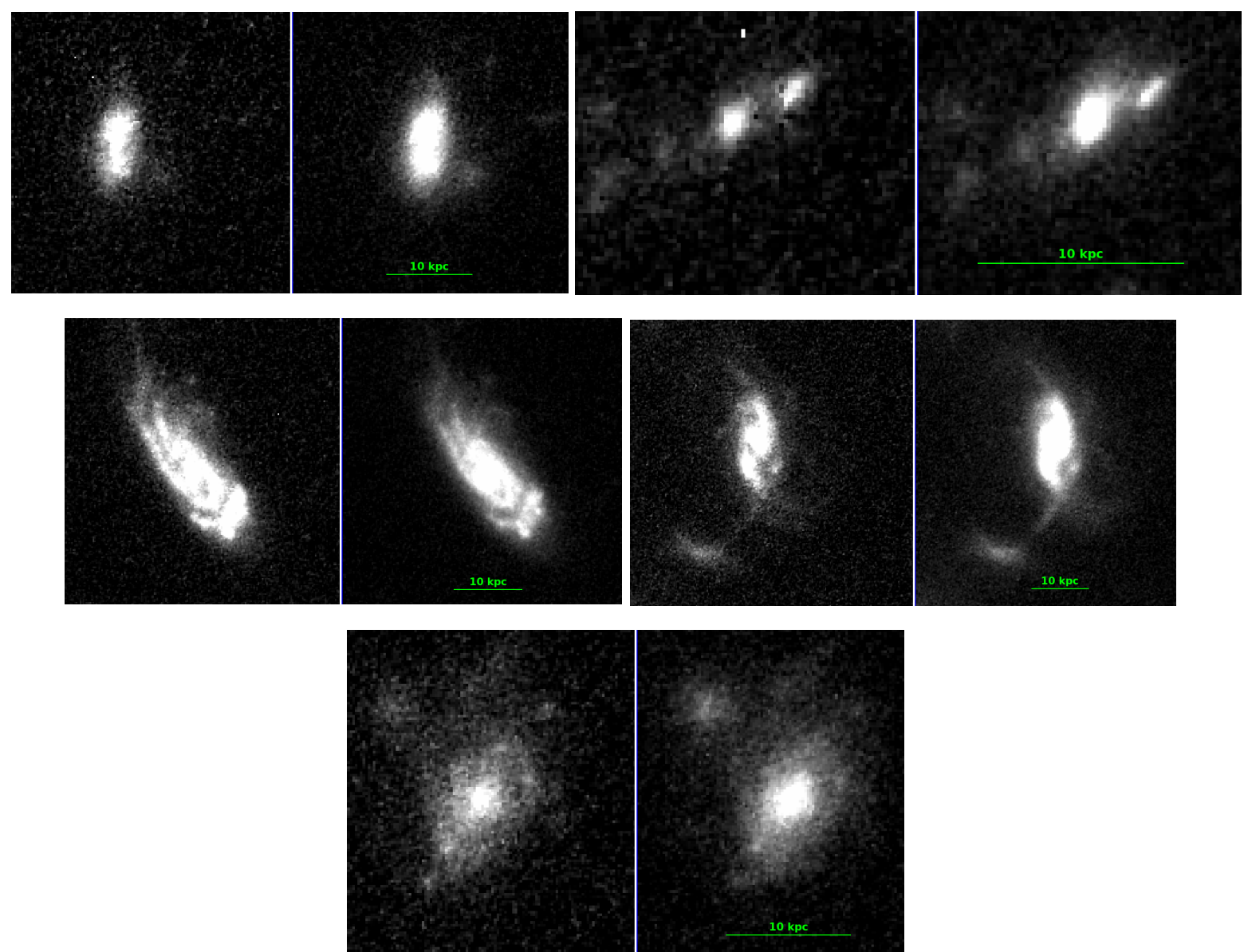

Fig. B.22. RX2248 $(z=0.348)$. Images are in $F 606 W$ and $F 814 W$. Row 1: Galaxies a and b. Row 2: Galaxies c and d. Row 3: Galaxy e. 